Graduate Theses, Dissertations, and Problem Reports

2021

Huntingtin aggregation at interfaces associated with membranes and organelles

Adewale Vincent Adegbuyiro

Follow this and additional works at: https://researchrepository.wvu.edu/etd

Part of the Biochemistry Commons, Biophysics Commons, and the Physical Chemistry Commons 


\title{
Huntingtin aggregation at interfaces associated with membranes and organelles
}

\author{
Adewale Adegbuyiro \\ Dissertation submitted to the \\ Eberly College of Arts and Science \\ at West Virginia University \\ in partial fulfillment of the requirements for the degree of \\ Doctor of Philosophy \\ in \\ Chemistry
}

Justin Legleiter, Ph.D., Committee Chairperson

Fabien Goulay, Ph.D.

Terry Gullion, Ph.D.

Blake Mertz, Ph.D.

David Smith, Ph.D.

C. Eugene Bennett Department of Chemistry

Morgantown, West Virginia

2021

Keywords: huntingtin, protein aggregation, mitochondria, mitochondrial membranes, amyloid, methionine oxidation, oligomers, fibrils. 


\begin{abstract}
Huntingtin aggregation at interfaces associated with membranes and organelles
\end{abstract}

\title{
Adewale Adegbuyiro
}

Huntington's Disease (HD) is a genetic neurodegenerative disease caused by the expansion of polyglutamine (polyQ) domain within the first exon (exon1) of the huntingtin (htt) protein. Due to this mutation within the polyQ domain, htt aggregates into various toxic species such as oligomers, fibrils, and other amorphous aggregates. While the aggregation of htt strongly correlates with polyQ length, other factors, e.g. interaction with membranes or organelles and posttranslational modifications (PTMs), modulate aggregation. The first $17 \mathrm{~N}$-terminal amino acids (Nt17) that precede the polyQ in httexon1 enhances aggregation and facilitated binding of htt to membranous organelles, promoting morphological changes and disfunction. In addition, several PTMs occur within Nt17, including oxidation. Here, mechanistic insights into the impact of mitochondrial association and oxidation of htt-exon1 on its aggregation are presented. To investigate how htt aggregation is altered in the presence of mitochondria, htt-exon1 with an expanded polyQ domain was exposed to mitochondria membrane mimics and mitochondria enriched fractions (MEFs). Mitochondrial membrane mimics significantly reduced htt aggregation into fibrils. However, inner mitochondrial membrane mimic (IMM) had a greater inhibitory effect on htt-exon 1 fibrillization compared with outer mitochondrial membrane mimic (OMM). Cardiolipin, a mitochondria-exclusive-lipid that is more abundant in IMM, played a primary role in altering htt aggregation. Similarly, MEFs suppressed fibril formation. With regard to htt oxidation, aggregation was inhibited by treatment with hydrogen peroxide $\left(\mathrm{H}_{2} \mathrm{O}_{2}\right)$ in a dose dependent manner. Specifically, fibril elongation and accumulation were prevented at high peroxide doses. The presence of total brain lipid extract (TBLE) vesicles did not alter the observed inverse relationship between htt aggregation and oxidant concentration. However, the combined effects of lipid presence and $\mathrm{H}_{2} \mathrm{O}_{2}$ treatment caused larger reduction in fibrillization compared with $\mathrm{H}_{2} \mathrm{O}_{2}$ treatment alone. Oxidation of htt-exon1 and/or lipid membranes altered aggregation patterns directly on a bilayer surface. Oxidation of both the protein and lipid promoted the appearance of plateau-like regions containing htt-exon1 aggregates. Collectively, these results demonstrate that htt-lipid interactions play significant roles in modulating htt aggregation pathways. 


\title{
Dedication
}

I dedicate this $\mathrm{PhD}$ dissertation to my parents

Lucas Olawale Adegbuyiro

Veronica Adunola Adegbuyiro

and to my siblings

\begin{abstract}
Adeola Adegbuyiro
Adejoke Adegbuyiro

Adedayo Adegbuyiro
\end{abstract}

and to the love of my life

Dr. Omotoyosi Akinbami

Your love, support, understanding, and prayers kept me going. 


\section{Acknowledgements}

I could not have achieved this feat alone, and it took a village's support to get me here.

My deepest appreciation goes to my PhD advisor, Dr. Justin Legleiter, who invested a lot in me to make me a well-rounded scientist. I acknowledge your input; your suggestions and the ideas shared to engage and challenge me. I am also grateful for your patience with me while I grow, and satisfy my scientific curiosities. The science I was exposed to under your tutelage was great, but so also were the taught life skills, that is, critical thinking and ideas presentation. I was not the most expressive person, but you are by far the one I have learnt the most from.

I am also grateful to my undergraduate advisor, Prof. E.T Akintayo, who introduced me to research, and to Dr. Nwokocha, who during my master's studies, taught me how to engage in independent research.

I extend my appreciation to my PhD defense committee members, Dr. Fabien Goulay, Dr. Terry Gullion, Dr. Blake Mertz, and Dr. David Smith for their time, advice, and encouragement, all so I can become a better scientist. Special appreciation to Dr. Stephen Valentine for all his help and time. I am also grateful for the opportunity and assistantship provided to me by West Virginia University and the C. Eugene Bennett Department of Chemistry to pursue a PhD program.

To my lab mates, I appreciate your contributions. Dr. Faezeh Sedighi, you were more than a lab mate, but also a sister who cared about my well-being and progress. Dr. Maxmore Chaibva, I remember and appreciate the talk we had when I started my PhD program. Dr. Albert Pilkington IV, I enjoyed and learnt a lot from our discussions, 
especially the late evening ones. Chathuranga Siriwardhana, you were always willing to help. Dr. Sharon Groover, thanks for the holiday gifts. I am also grateful to Adam Skeens, Dr. Maryssa Beasley and Alyssa Stonebraker for their feedbacks and contributions.

My heartfelt gratitude goes to my family: Theresa Olatoye, Seun Olobatuyi, Adoide Akinyemi, Adoide Olajide. To my friends: Dr. Eric Sefah, Dr. Gideon Gogovi, Ayodeji Funso, Akintunde Akinola, Matthew Bamidele, Dr. Ogunyemi Oderinlo, Daniella Munezero, Danielle Hills, Phuong Minh Do, and Stephanie Agba. You made life outside of the lab enjoyable.

A special acknowledgement to the love of my life- Dr. Omotoyosi Akinbami. I am grateful for your love, patience, understanding, prayers, and support. You have stood by me and showed me your unwavering love. To my parents, Lucas Olawale Adegbuyiro and Veronica Adunola Adegbuyiro, you have always believed in me, and you invested in my dreams. To Adeola, Adejoke and Adedayo, I could not have asked for a more supportive bunch. You are the best siblings ever.

Above all, I thank God for the grace to do all that I have accomplished. 


\section{Table of Contents}

$\begin{array}{ll}\text { Abstract } & \text { ii }\end{array}$

Dedication

Acknowledgments $\quad$ iv

Table of Contents $\quad$ vi

List of Figures $\quad$ x

List of Tables $\quad$ xiii

List of Symbols and abbreviations $\quad$ xiv

1. Introduction: Protein aggregation in Huntington's and neurodegenerative $\begin{array}{ll}\text { diseases } & 1\end{array}$

1.1 Neurodegenerative diseases and protein aggregation 1

1.2 Proteins implicated in amyloid-based neurodegenerative diseases 3

1.3 Huntingtin aggregation in Huntington's Disease $\quad 4$

1.4 Factors that modulate huntingtin aggregation $\quad 7$

1.4.1 Flanking sequences influence htt aggregation $\quad 7$

1.4.2 Posttranslational modifications in Nt17 alter htt aggregation 9

1.4.3 Oxidation of $\mathrm{Nt17} \quad 10$

1.4.4 Interaction of huntingtin with membranes 11

1.5 Dissertation rationale and chapters summary 13

$\begin{array}{ll}1.6 \text { References } & 14\end{array}$ 
2.1 Introduction: Mutant htt aggregates, localizes to organelles and promotes $\begin{array}{ll}\text { organelles dysfunction } & 25\end{array}$

$\begin{array}{ll}2.2 \text { Methods } & 28\end{array}$

2.2.1 Purification of GST-htt exon1 fusion protein 28

2.2.2 Isolation of Mitochondrial-Enriched Fractions 28

$\begin{array}{ll}\text { 2.2.3 Preparation of Lipid Vesicles } & 29\end{array}$

2.2.4 Thioflavin T Fluorescence Assay 29

2.2.5 Atomic Force Microscopy (AFM) 30

2.2.6 Filter Retardation and Dot Blot Assay 31

2.2.7. Monomer loss assay 32

2.3 Results 33

2.3.1 Mitochondrial membrane mimics reduce htt fibril formation 33

$\begin{array}{ll}\text { 2.3.2 Cardiolipin impacts htt aggregation } & 37\end{array}$

2.3.3 $\mathrm{Htt}$ aggregation varies on the surface of OMM and IMM mimics $\quad 39$

2.3.4 OMM, IMM, and CL containing vesicles do not destabilize

preformed htt fibrils 43

2.3.5 Mitochondrial-enriched fractions inhibit fibril formation 45

2.3.6 Mitochondria influence htt oligomer formation 53 
2.3.7 Mitochondria-enriched fractions do not destabilize preformed htt fibrils

2.4 Discussion

3. Oxidation promotes distinct huntingtin aggregates in the presence and absence of membranes

3.1 Introduction: Nt17 has posttranslational modification sites and modulates

htt aggregation and organelles interaction

3.2 Methods

3.2.1 Preparation of synthetic peptides

3.2.2 Purification of GST-htt exon1 fusion protein 76

3.2.3 Preparation of Lipid Vesicles

3.2.4 Thioflavin T (ThT) Assay

77

3.2.5 Polydiacetylene (PDA) Vesicle Binding Assay

3.2.6 Atomic Force Microscopy (AFM 
3.3.3 Oxidation reduces htt fibrillization in the presence of lipids

3.3.4 Oxidative environment influence oligomerization in the presence of lipid vesicles

3.3.5 Oxidation alter htt aggregation directly on the TBLE bilayer surface

4.1 Introduction

4.3 Investigation of the role of oxidized lipids of mitochondria on htt aggregation 


\section{List of Figures}

1.1 Energy landscape of protein folding and misfolding for amyloid forming proteins

1.2 Huntingtin protein

1.3 PolyQ aggregation showing different pathways

1.4 Nt17 helical wheel 10

2.1 Raw ThT signals associated with the different lipid vesicles 33

2.2 Mitochondrial membrane mimics inhibit fibril formation

2.3 Dose dependent impact of $(A) \mathrm{OMM}$ and $(B)$ IMM vesicles on htt-exon1 (46Q)

2.4 Control ex situ AFM images of backgrounds associated with the lipid systems deposited on mica and used to study htt aggregation

2.5 Cardiolipin impacts htt aggregation

2.6 Huntingtin aggregation varies on the surfaces of OMM and IMM membrane mimics

2.7 Additional in situ AFM images of supported (A) OMM and (B) IMM bilayers exposed to $10 \mu \mathrm{M}$ htt-exon1(46Q)

2.8 OMM, IMM, and CL do not disaggregate preformed htt fibrils

2.9 The impact of MEFs derived from murine brains on htt-exon1(46Q) aggregation

2.10 The impact of MEFs derived from bovine liver on htt-exon1 (46Q) aggregation 
2.11 Analysis of identifiable mitochondria populations incubated with (orange) and without (red) htt-exon1(46Q)

2.12 Immunochemical analysis of murine brain derived MEFs with and without htt-exon1(46Q) at various time points

2.13 Immunochemical analysis of htt-exon1(46Q) without and with bovine liver-derived MEFs at various time points

2.14 Control ThT assay demonstrating the impact of residual material in the supernatant of centrifuged MEFs on htt-exon1(46Q) $(20 \mu \mathrm{M})$ fibril formation

2.15 Murine-derived brain MEFs alter oligomer morphology

2.16 Analysis of oligomers formed by htt-exon1(46Q) in the presence and absence of MEFs derived from bovine liver

2.17 MEFs do not destabilize preformed htt fibrils

3.1 The impact of $\mathrm{H}_{2} \mathrm{O}_{2}$ on $\mathrm{Nt}^{17} \mathrm{Q}_{35} \mathrm{P}_{10}$ aggregation as assessed by a ThT assay

3.2 Oxidation alters the aggregation of $\mathrm{htt}$

3.3 Oxidation of htt-exon1 (46Q) promoted oligomerization

3.4 ThT analysis of oxidized and unoxidized $\mathrm{Nt}^{17}-\mathrm{Q}_{35}-\mathrm{P}_{10}$ peptide aggregation in the presence of lipid vesicles

3.5 PDA/TBLE lipid binding assay of htt-exon1 (46Q) exposed to no or various $\mathrm{H}_{2} \mathrm{O}_{2}$ concentrations

3.7 Peripheral features formed around htt-exon1(46Q) at extremely high 
oxidant concentration

3.8 Oxidation of htt-exon1(46Q) promoted oligomerization in the presence of TBLE

3.9 TBLE bilayers stability in the presence of $\mathrm{H}_{2} \mathrm{O}_{2}$

3.10 Oxidation alters htt aggregation directly on the TBLE bilayer surface $\quad 97$

3.11 Correlation plots showing mean length and mean height of htt aggregates on the surface of TBLE bilayers

4.1 Determination of effect of oxidized lipids of mitochondria on htt aggregation

4.2 Comparison of $\mathrm{Nt17-} \mathrm{Q}_{35}-\mathrm{P}_{10}-\mathrm{KK}$ aggregation on mica with different micromolecular crowders in the aqueous phase

4.3 Comparison of htt-exon1(46Q) aggregation on supported TBLE bilayers with different macromolecular crowders in the aqueous phase

4.4 NUCB1 inhibited the aggregation of htt-exon1

4.5 NUCB1 inhibited the aggregation of $\mathrm{Nt}^{17}-\mathrm{Q}_{35}-\mathrm{P}_{10}-\mathrm{KK}$ 


\section{List of Tables}

1.1 Polyglutamine disorders 


\section{List of symbols and abbreviations}
AD
Alzheimer's disease
AFM
atomic force microscopy
$\mathrm{CL}$
cardiolipin
DMSO
dimethyl sulfoxide
DRP1
dynamin related protein 1
ER
endoplasmic reticulum
GST
glutathione S-transferase
$\mathrm{H}_{2} \mathrm{O}_{2}$
hydrogen peroxide
HD
Huntington's disease
HFIP
hexafluoroisopropanol
$\mathrm{Htt}$
huntingtin
Htt-exon1(46Q) first exon of htt containing 46 glutamine repeats
IMM
inner mitochondrial membrane
IPTG
isopropyl- $\beta$-d-1-thiogalactopyranoside
LPG
lysophosphatidylglycerol
LPC
lysophosphatidylcholine
M1
methionine- 1
M8
methionine- 8
MEF
mitochondria-enriched fraction
NCM
nitrocellulose membrane
Nt17
$17 \mathrm{~N}$-terminal amino acids of huntingtin
$\mathrm{N}^{17}-\mathrm{Q}_{35}-\mathrm{P}_{10}-\mathrm{KK} \quad$ synthetic polyQ peptide with 35 glutamines and 10 prolines and two lysines 


$\begin{array}{ll}\text { OMM } & \text { outer mitochondrial membrane } \\ \text { Ox. TBLE } & \text { oxidized total brain lipid extracts } \\ \text { PBS } & \text { phosphate buffered saline } \\ \text { PD } & \text { Parkinson's disease } \\ \text { PC } & \text { phosphocholine } \\ \text { PDA } & \text { polydiacetylene } \\ \text { PE } & \text { phosphatidylethanolamine } \\ \text { PI } & \text { phosphatidylinositol } \\ \text { PolyP } & \text { polyproline } \\ \text { PolyQ } & \text { polyglutamine } \\ \text { POPG } & \text { 1-palmitoyl-2-oleoyl-sn-glycero-3-(phosphor-rac-(1-glycerol)) } \\ \text { POPC } & \text { 1-palmitoyl-2-oleoyl-sn-glycero-3-phosphocholine } \\ \text { POPS } & \text { 1-palmitoyl-2-oleoyl-sn-glycero-3-phospho-L-serine } \\ \text { PS } & \text { thioflavin T } \\ \text { PTM } & \text { total brain lipid extract } \\ \text { RMS } & \text { posttranslational modification } \\ \text { ROS } & \text { root mean square } \\ \text { SDS } & \text { reactive oxygen species } \\ \text { SDS-PAGE } & \text { sodium dodecyl sulfate } \\ \text { SEM } & \text { standium dodecyl sulfate polyacrylamide gel electrophoresis } \\ \text { TBLE } & \text { thA }\end{array}$


yeast artificial chromosome 


\section{Introduction: Protein aggregation in Huntington's and neurodegenerative diseases}

\subsection{Neurodegenerative diseases and protein aggregation}

A great number of neurodegenerative diseases are commonly characterized by proteinaceous deposits comprised predominately of amyloid, broadly defined as protein aggregates with a fibrillar morphology and a cross $\beta$-sheet secondary structure. ${ }^{1}$ These diseases include Alzheimer's, Parkinson's, and Huntington's Disease (HD). Similar to protein folding, the formation and deposition of protein aggregates is a free energy driven process (Fig. 1). Encoded in the amino acids sequence, nascent high enthalpy proteins fold into enthalpically-favorable three-dimensional functional or native conformations via folding intermediates that may prolong the folding process by acting as kinetic traps. ${ }^{2}$ The free energy landscape based on the various conformations available to a nascent protein promotes a pathway toward the native protein structure. However, proteins can become trapped in enthalpically unfavorable local energy minima, which represent misfolded states along the rugged free energy landscape. Unlike the native state, misfolded proteins associate to form aggregates due to increased intermolecular interactions between exposed hydrophobic residues. The initial aggregates formed typically are small, transient oligomeric species capable of reorganizing into more stable $\beta$-sheet rich oligomers, or highly structured, insoluble $\beta$-sheet rich amyloid fibrils. ${ }^{3-5}$ Amyloid fibrils display enhanced enthalpic stability due to a network of intermolecular hydrogen bonds holding the $\beta$-sheets together (Fig. 1). Alternatively, misfolded proteins can also form less compact amorphous aggregates. These different aggregate species accumulate as the deposits, plaques, and 


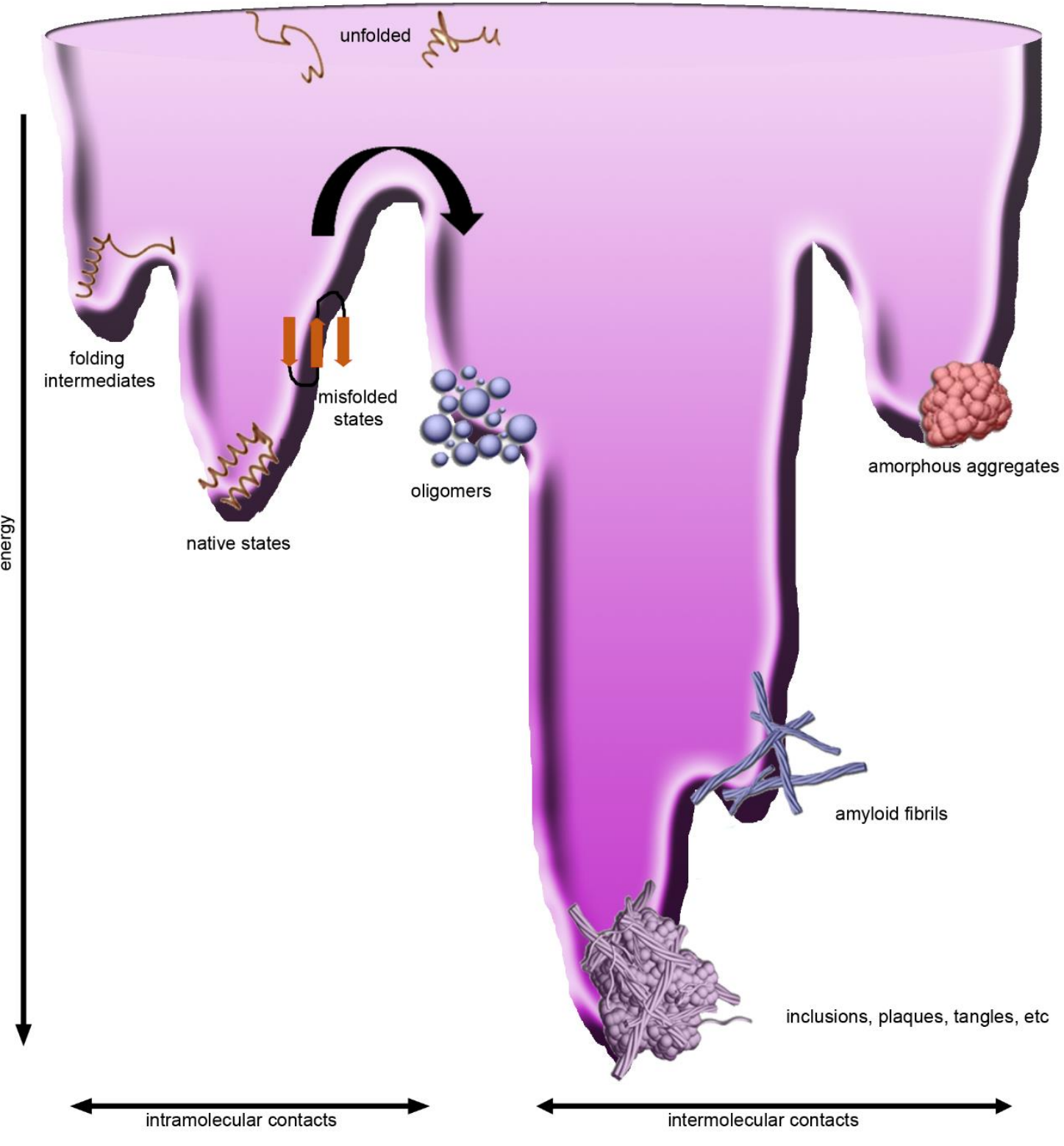

Figure 1. Energy landscape of protein folding and misfolding for amyloid-forming proteins. Proteins fold into their energetically favorable native states based on a rugged free energy landscape. The rugged energy landscape of protein folding also allow for proteins to be trapped in local energy minima in a misfolded or partially folded state. Misfolded proteins can proceed to form amorphous aggregates, or fibrils through oligomers. Accumulation of aggregates form low energy plaques or inclusions. 
inclusions in neurons that are associated with the emergence of neurodegenerative diseases.

\subsection{Proteins implicated in amyloid-based neurodegenerative diseases}

Neurodegenerative diseases associated with amyloid formation share several common features. Aggregate formation is a consequence of protein misfolding and is triggered by conditions such as proteolysis, ageing, mutations, and other modifications. However, specific proteins are implicated in different neurodegenerative diseases.

Table 1.1 Polyglutamine Disorders.

\begin{tabular}{|l|l|l|l|}
\hline disease & common name & protein & polyQ $^{\text {a }}$ \\
\hline Huntington's Disease (HD) & huntingtin & $36-100$ \\
\hline $\begin{array}{l}\text { Spinobulbar muscular } \\
\text { atrophy (SBMA) }\end{array}$ & Kennedy's disease & androgen receptor & $38-65$ \\
\hline $\begin{array}{l}\text { Dentatorubral-pallidoluysian } \\
\text { atrophy (DRPLA) }\end{array}$ & Haw River syndrome & atrophin-1 & $49-88$ \\
\hline $\begin{array}{l}\text { Spinocerebellar ataxia type } \\
1 \text { (SCA1) }\end{array}$ & ataxin-1 & $39-88$ \\
\hline $\begin{array}{l}\text { Spinocerebellar ataxia type } \\
\text { 2 (SCA2) }\end{array}$ & ataxin-2 & $33-77$ \\
\hline $\begin{array}{l}\text { Spinocerebellar ataxia type } \\
\text { 3 (SCA3) }\end{array}$ & Machado-Joseph & ataxin-3 & $55-86$ \\
\hline $\begin{array}{l}\text { Spinocerebellar ataxia type } \\
6 \text { (SCA6) }\end{array}$ & CACNA1A & $21-33$ \\
\hline $\begin{array}{l}\text { Spinocerebellar ataxia type } \\
7 \text { (SCA7) }\end{array}$ & ataxin-7 & $38-120$ \\
\hline $\begin{array}{l}\text { Spinocerebellar ataxia type } \\
12 \text { (SCA12) }\end{array}$ & PPP2R2B & $66-78$ \\
\hline $\begin{array}{l}\text { Spinocerebellar ataxia type } \\
17 \text { (SCA17) }\end{array}$ & TATA-box binding & $47-63$ \\
\hline
\end{tabular}

andicates the typical pathogenic polyQ range associated with each disease. 
For example, neurofibrillary tangles of tau and extra-neuronal senile plaques of amyloid- $\beta$ are hallmarks of Alzheimer's Disease (AD); while Lewy bodies of $\alpha$-synuclein are observed in Parkinson's Disease (PD). A subset of amyloid-based neurodegenerative diseases is associated with expansion of a repeat glutamine (Q) stretch in specific proteins that promote aggregation. To date, ten polyglutamine (polyQ) disorders have been identified (table 1). ${ }^{6}$ There exists a variety of proteins associated with the different polyQ disorders with implications for the specific neuronal populations impacted. For each of the identified polyQ diseases, there exist a protein specific threshold of polyQ elongation associated with pathogenesis (table 1). For all these diseases, expansion beyond this critical threshold is correlated with disease severity and aggregation.

\subsection{Huntingtin aggregation in Huntington's Disease}

$\mathrm{HD}$ is an inherited autosomal dominant disorder caused by expansion of a polyQ domain near the $\mathrm{N}$-terminus of the huntingtin protein (htt). Clinically, patients present with a triad of movement disorder (chorea), psychiatric manifestation, and cognitive loss. ${ }^{7}$ While the typical length of the polyQ stretch in htt is $\sim 17-20$ repeat units, the critical threshold for HD is expansion beyond 35 repeat glutamine residues ${ }^{8}$ (Table 1). The expansion of the polyQ (polyQ length) also strongly correlates with the age of onset and severity of the disease.$^{8-10}$ For example, the average age of onset with a polyQ repeat length of $\sim 44$ is about 35 years. ${ }^{11}$ In contrast, juvenile forms of HD (symptoms observed before 20 years of age) have an average repeat length of $\sim 60 \mathrm{Q} .{ }^{11}$

$\mathrm{Htt}$ is a large, multifunctional protein $(\sim 350 \mathrm{KDa}$, Fig. 2) typically found in the cytoplasm. Htt has been associated with axonal trafficking of vesicles and organelles, regulation of gene transcription, protein handling at the ER-Golgi complex, and 
antiapoptotic activity. ${ }^{12}$ Of interest for HD is the first exon of htt (htt-exon1, Fig.2) and other $\mathrm{N}$-terminal fragments that contains the polyQ domain. ${ }^{13-15}$ Aggregates of $\mathrm{N}$-terminal fragments containing a polyQ expansion are found in the affected areas of the brain in $\mathrm{HD}^{15}$ and are produced prior to pathogenesis through protease activities. ${ }^{16,17}$ Proteolytic products of htt form a variety of aggregate species. ${ }^{18,19}$

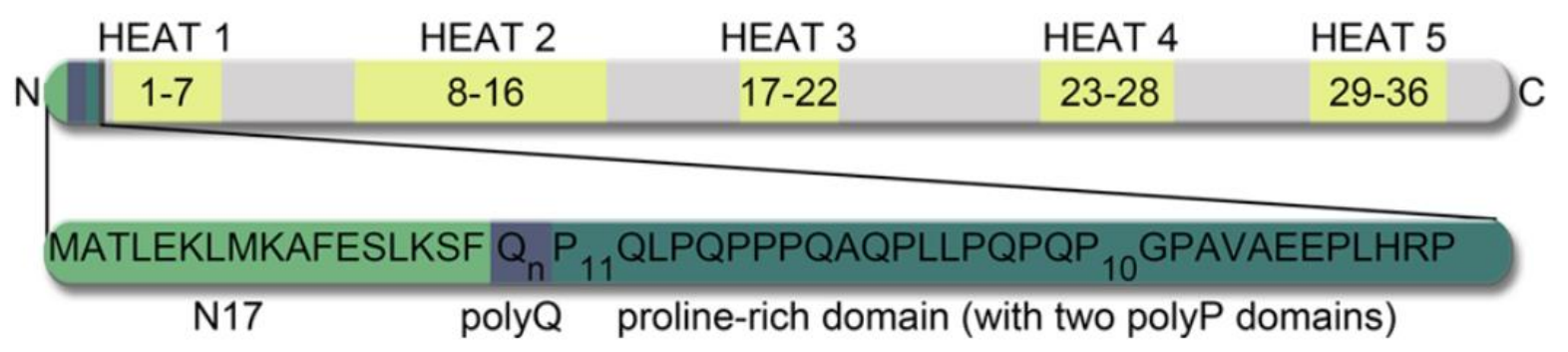

Figure 2. Huntingtin protein. Full length htt with 5 HEAT repeats (top). The inset at the $N$-terminal of huntingtin is the first exon (htt-exon1) with three domains (bottom) namely: $N+17$ ( $N$ terminal amino acids), polyglutamine and polyproline.

The aggregation mechanism of huntingtin is similar to that of typical amyloid (Fig. 1 and 3). In a classical fibril formation pathway, fibril nucleation occurs either directly from htt monomers or through oligomeric intermediates. While these pathways are not mutually exclusive, polyQ length tend to shift the dynamics, with longer lengths favoring fibril formation from monomeric htt. ${ }^{20-22}$ Additional complexity in aggregation derives from competing aggregation mechanisms off-pathway to fibril formation that lead to amorphous aggregates. While predominately comprised of fibrils, all aggregate types can be trapped in larger inclusion bodies that are found ubiquitously in mouse models ${ }^{23}$ and in the brains of HD patients. ${ }^{24}$ The heterogeneity in the make-up of inclusions ${ }^{24}$ further suggests that competing pathways lead to their formation. Regardless of the path taken, aggregates of 
htt display toxic gain of function, ${ }^{25}$ as oligomers, ${ }^{26,27}$ fibrils, ${ }^{28,29}$ and inclusions ${ }^{30}$ have all been linked directly to toxicity. Oligomers are present in brains of different HD disease mouse models. Using a combination of Electron Microscopy and Atomic Force Microscopy, analysis of the lysate obtained from the cortex of R6/2 mice revealed that oligomers were present early, in 2-week-old mice. ${ }^{31}$ Similar globular aggregates were obtained from 2 months old Hdh150 knock-in mouse models. Interestingly, these aggregates are similar to those obtained in vitro, from htt-exon1 with 46 and 51 glutamine repeats. ${ }^{31}$ The toxicity of fibrils have been demonstrated using various polyQ proteins. ${ }^{28}$

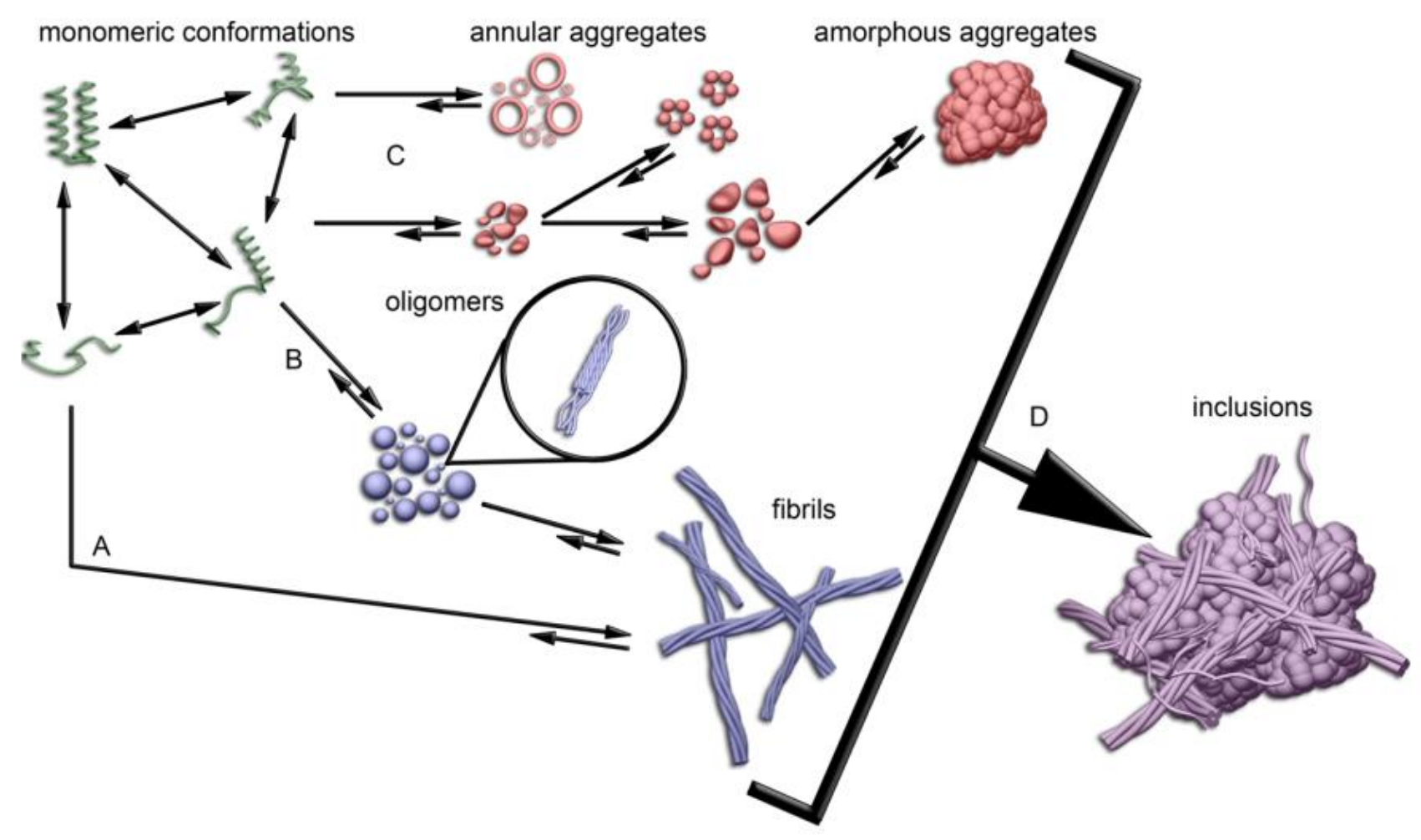

Figure 3: PolyQ aggregation showing different pathways. (A) Nucleation of monomeric htt to form fibrils. (B) Nt17 promotion of htt monomers aggregation into fibrils via oligomeric intermediates. (C) Formation of annular and amorphous aggregates. (D) Accumulation of various htt aggregates into inclusion bodies. 
The fibrils obtained from different regions of an $R 6 / 2$ mouse model displayed various degrees of toxicity when transfected into neuro2a cells. ${ }^{32}$ Similarly, when httexon1 fibrils were introduced to SH-SY5Y cells, cell viability decreased significantly. ${ }^{29}$ Indeed, htt aggregation is crucial in HD, and polyQ expansion drives a variety of aggregation pathways. However, beyond the polyQ, many other factors alter htt aggregation.

\subsection{Factors that modulate huntingtin aggregation}

With toxicity directly associated with htt aggregation, understanding the complex aggregation process, and the resulting heterogeneous mixture of aggregates species can provide insight into the development of HD. However, the htt aggregation process is highly dependent on various factors, including protein context and protein environment. For example, protein context of an aggregation prone polyQ domain is thought to underlie the variation in the critical poly $Q$ expansion threshold observed across different poly $Q$ diseases. ${ }^{33-41}$ Specifically for htt, different $\mathrm{N}$-terminal fractions of htt can lead to unique aggregate species, ${ }^{42}$ and protein sequences directly flanking the polyQ domain strongly influences polyQ aggregation. ${ }^{35,37,38,43-45}$ Similar to other amyloids, htt aggregation is also influenced by its environmental conditions, i.e. $\mathrm{pH}$, ionic strength, crowding, and membranes.

1.4.1 Flanking sequences influence htt aggregation. Within htt-exon1, upstream of the polyQ tract are the first $17 \mathrm{~N}$-terminal amino acids (Nt17) that act as a lipid binding motif ${ }^{39,46}$ and contains several post-translational modification (PTM) sites (Fig. 2). ${ }^{47,48}$ Cterminal to the polyQ is the proline-rich region, which contains two distinct polyproline (polyP) tracts (Fig. 2). These polyQ flanking sequences (Nt17 and polyP) have contrasting 
effects on htt aggregation. Nt17 promotes aggregation by facilitating oligomerization by forming amphipathic $\alpha$-helices that intermolecularly associate; ${ }^{33,35}$ polyP promotes aggregation-incompetent monomeric species by extending a PPII-type helical structure into the polyQ domain. ${ }^{49}$

The presence of polyP suppresses htt aggregation. ${ }^{35,36,49-51}$ With a PPII helical structure $^{36,52}$ that extends into the polyQ tract in crystal structures, ${ }^{52}$ polyP hinders $\beta$ sheet rich states of polyQ as revealed by molecular dynamics simulations. ${ }^{36}$ Specifically, addition of a C-terminal polyP sequence to synthetic polyQ peptides reduces aggregation in vitro. ${ }^{49}$ In fact, the presence of the polyP in synthetic htt-exon1 mimic was sufficient to alter the length of polyQ required for fibrillization. ${ }^{43,49} \mathrm{~A}$ similar observation has been demonstrated in yeast cells ${ }^{38,51}$ where longer polyglutamine length is required to counter the ability of polyP to hinder aggregation, ${ }^{51}$ and presence or absence of polyP is sufficient to convert between benign and toxic aggregate species in yeasts. ${ }^{38}$

Nt17 drives early steps in fibril formation ${ }^{35,39,53-57}$ through intermolecular interactions. Structurally, Nt17 adopts multiple configurations in solution that vary from a predominantly intrinsically disordered state as determined by nuclear magnetic resonance $(\mathrm{NMR}){ }^{35}$ to a significantly a-helical structure revealed by circular dichroism. ${ }^{35,58,59}$ This ability to adopt an amphipathic $\alpha$-helical structure facilitates intermolecular association to form $\alpha$-helix rich oligomer intermediates. ${ }^{60}$ Specifically, when oligomerization, which is often an early event in htt fibrillization, is promoted, the polyQ domains of neighboring htt proteins come into close proximity, lowering the barrier to nucleation. ${ }^{33,35,60}$ In contrast to polyP, this influence of $\mathrm{Nt17}$ on aggregation of htt is not dependent on its position. The insertion of $\mathrm{Nt17}$ to either the $\mathrm{N}$ - or $\mathrm{C}$-terminal of polyQ 
resulted in increased aggregation. ${ }^{35}$ Indeed, the mechanism through which this binding domain alters huntingtin aggregation lies in its ability and availability to interact with other $\mathrm{Nt} 17$ domains. The removal of $\mathrm{Nt} 17$ from either htt-exon $1^{61}$ or its mimics ${ }^{35}$ resulted in significant reduction in aggregation or a delayed monomer loss. In addition, through interaction with other proteins, the Nt17 loses its ability to drive huntingtin aggregation. ${ }^{61,62}$

Nt17 may also be involved in intramolecular interactions with other regions of httexon1 to promote different conformations. Modulated by interactions with other proteins, and with the polyQ acting as a hinge, intramolecular interactions between $\mathrm{Nt17}$ and the polyP regions of htt-exon1 is possible. ${ }^{63}$ Based on investigations using FRET, the nonpathogenic length polyQ domains provide the necessary flexibility for $\mathrm{Nt17}$ and polyP to interact; however, once polyQ domains reach the expansion threshold associated with $H D$, the poly $Q$ domain adopts a structure that prevents intramolecular association between Nt17 and polyP. This observation has led to the "rusty hinge" hypothesis for htt aggregation. This hypothesis states that the threshold associated with the disease arises from the expanded polyQ domain's lost flexibility, thereby freeing Nt17 from intramolecular associations and promoting its ability to form intermolecular associations that initiate aggregation. Based on its importance in initiating aggregation, Nt17 has emerged as a therapeutic target.

1.4.2 Posttranslational modifications in Nt17 alter htt aggregation. Nt17 is a site for several PTMs that impact htt function, localization, aggregation, and toxicity. ${ }^{64-68}$ Residues within Nt17 that are readily modified include lysine, threonine, serine and methionine (Fig 4). PTMs within Nt17 include phosphorylation, ${ }^{65,69,70}$ acetylation, ${ }^{71}$ ubiquitination, SUMOylation ${ }^{66}$ and oxidation. ${ }^{72}$ Phosphorylation of $\mathrm{Nt} 17$ is associated with 
reduced aggregation ${ }^{70,73}$ and toxicity ${ }^{65,67,69,70}$ Phosphorylation likely inhibits fibrillization by destabilizing the formation of the $\mathrm{Nt17} \alpha$-helix associated with initiating aggregation. ${ }^{73,74}$ SUMOYlation of huntingtin modulates htt aggregation and toxicity by blocking the formation of SDS insoluble aggregates in cells ${ }^{66}$ and in vitro. ${ }^{47}$ Acetylation of lysine residues within Nt17 occurs in cells ${ }^{71}$ and in vitro. ${ }^{48}$ This modification inhibits fibrillization of htt possibly by promoting the formation of unique oligomers that may be incapable of transitioning into fibrils, or by stabilizing the oligomeric aggregates and thereby delaying fibrillization. ${ }^{48}$

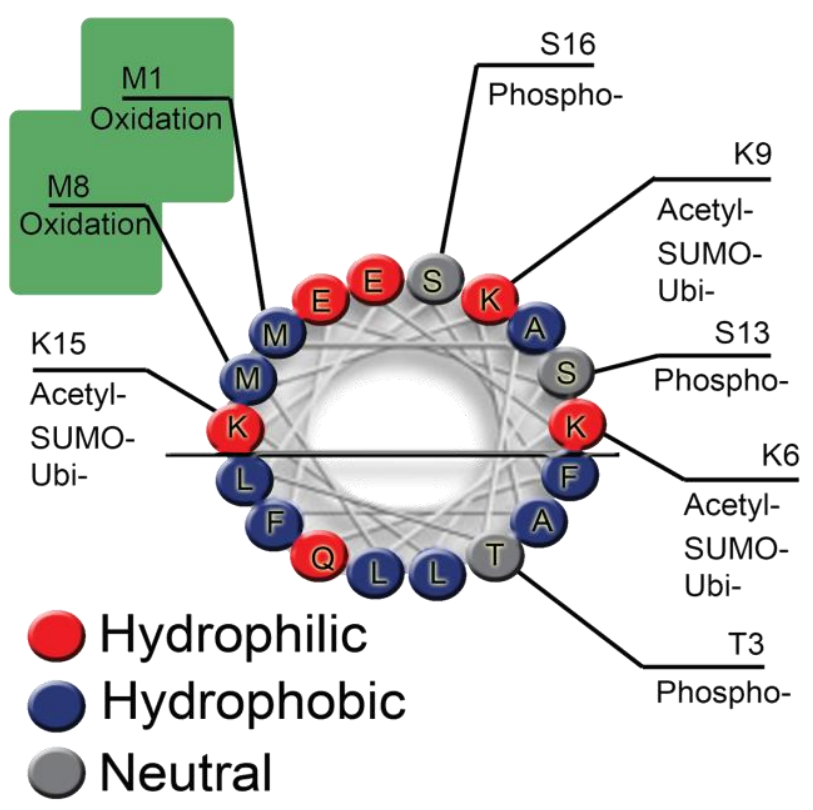

Figure 4: Nt17 helical wheel. The 17 amino acid residues of Nt17 and the first glutamine of the poly $Q$ domain showing the sites of various post translational modifications that are possible within Nt17.

1.4.3 Oxidation of Nt17. Oxidative stress which is caused by imbalance of redox states has been implicated in many neurodegenerative diseases. ${ }^{75-77}$ Oxidation of htt, 
especially within $\mathrm{Nt} 17,{ }^{72,78}$ is another PTM that alters htt aggregation. There are two oxidizable methionine residues (M1 and M8) within Nt17 (Fig. 2 and 4). The oxidation of M8 in htt-exon1 inhibits fibril formation. ${ }^{35,79,80}$ In an NMR study of htt-exon1 mimic containing Nt17 and 10 glutamine repeats only, peroxide oxidation of M8 catalyzed by titanium oxide nanoparticles stabilized aggregation-incompetent conformers by 4 folds. ${ }^{79}$ Precisely, the oxidation of the side chain of methionine to methionine sulfoxide prevented the aggregation-induced broadening of NMR signal when compared with controls. In contrast, without significant effect on the total amount of aggregates formed, postaggregation oxidation of M8 in htt-exon1 favored the occurrence of larger aggregates through the accumulation of pre-oxidation formed aggregates. ${ }^{78}$

1.4.4 Interaction of huntingtin with membranes. Approximately half of htt present in the cytoplasm is associated with lipid membranes, including the plasma membrane. ${ }^{81}$ $\mathrm{Htt}$ localizes to a variety of membranous organelles causing their disruption and distortion of their membranes, and ultimately their dysfunction. ${ }^{82-87}$ Both full length and $\mathrm{N}$-terminal fragments of htt interact with membranous structures such as $E R,{ }^{58,88}$ mitochondria, ${ }^{82,83,89,90}$ the golgi apparatus, ${ }^{91}$ and the nuclear envelope.${ }^{91}$ The interaction of htt with membranous surfaces have pathological implications. For example, various membranous structures are incorporated into inclusion bodies in cell lines expressing mutant $\mathrm{N}$-terminal fragments. ${ }^{92,93}$ In particular, the association of $\mathrm{N}$-terminal fragments of mutant htt with mitochondria is linked to mitochondrial dysfunction. ${ }^{82,94-97}$ Mutant huntingtin unfavorably alters mitochondrial membrane potential ${ }^{83}$ and promotes mitochondrial fission. ${ }^{95}$ 
Similar to disease severity and aggregation, the deleterious effect of mutant htt on membranes are also polyQ length dependent. ${ }^{98-100}$ When vesicles made from various phospholipids were probed for huntingtin interaction using brain cytosols obtained from mice with wild type or mutant huntingtin, mutant htt associated more with phospholipids. ${ }^{101}$ Similarly, AFM studies revealed that htt-exon1 disruption of bilayers formed from total brain lipid extract (TBLE) was enhanced with increasing polyQ length. ${ }^{99}$ In addition, exposure of htt-exon1 to TBLE bilayers altered the local mechanical properties of the membrane. ${ }^{99}$ These studies further provide a hypothesis with regards to the organelle dysfunction or impairment of membrane trafficking that is associated with mutant $h t t,{ }^{82}$ that is, htt containing expanded polyQ domains directly damage membranes disrupting their normal function.

The direct interaction of $\mathrm{N}$-terminal fragments of htt to lipid membranes is facilitated by Nt17. ${ }^{37,102}$ This ability is regulated by PTMs. Lysine acetylation within Nt17 reduces the affinity of htt-exon1 for lipid bilayers, reducing htt's ability to damage membranes and resulting in reduced cellular toxicity. ${ }^{48}$ Similarly, interaction of htt-exon1 with supported TBLE was inhibited when htt exon1 was SUMOylated. ${ }^{47}$ Oxidation of $\mathrm{Nt} 17$ triggers release of htt from the ER membrane, resulting in htt being trafficked to the nucleus. In vitro, oxidation of methionine in a synthetic htt peptide reduced its binding to a 3:1 micellar mix of lysophosphatidylcholine (LPC) and lysophosphatidylglycerol (LPG) by 3-4 folds, probably due to increased repulsion between methionine sulfoxide and the lipid head groups. ${ }^{80}$ Upon interaction with membranes, htt aggregation is altered; however, the specific impact is dependent on membrane composition. ${ }^{81,98}$ Some lipid systems (POPC, POPG, POPS, POPC/POPS) increased fibrilization;103-105 other lipid systems (TBLE, 
POPE) inhibit aggregation. ${ }^{99,105-107}$ Nevertheless, unique aggregation mechanisms are associated with htt aggregation on membrane surfaces. For example, POPC/POPS membranes create high local htt concentrations, creating nucleation sites and promoting aggregation. ${ }^{80,104}$ Both of these lipids also alter the $\alpha$-helical content of htt. ${ }^{58}$

\subsection{Dissertation rationale and chapters summary}

Aggregation of mutant huntingtin is associated with HD. The aggregation process of mutant htt is greatly influenced by membrane association and chemical modifications. In addition, mutant htt that are prone to aggregation are associated with organelle dysfunction. Understanding the impact of cellular factors (i.e., organelle surfaces) and chemical modifications on htt aggregation can reveal novel therapeutic approaches toward treating HD. In Chapter 2, we investigated htt aggregation in the presence of mitochondrial surfaces using a variety of systems. Mitochondrial surfaces suppressed fibril formation and unique aggregation patterns directly on membranes. An important role of cardiolipin, a lipid predominately found mitochondrial membranes, in suppressing htt aggregation was revealed. In Chapter 3 , the impact of htt oxidation, via $\mathrm{H}_{2} \mathrm{O}_{2}$ treatments, on aggregation in the absence and presence of lipid membranes was determined. Oxidation suppressed htt aggregation under both conditions. However, oxidation of lipids also impacted the interaction between htt and membranes. Finally, Chapter 4 expands upon these findings and how they can be applied to a better understanding of HD. 


\subsection{References}

(1) Dugger, B. N., and Dickson, D. W. (2017) Pathology of neurodegenerative diseases. Cold Spring Harbor Perspectives in Biology 9, 1-22.

(2) Jahn, T. R., and Radford, S. E. (2005) The Yin and Yang of protein folding. FEBS Journal 272, 5962-5970.

(3) Cheon, M., Chang, I., Mohanty, S., Luheshi, L. M., Dobson, C. M., Vendruscolo, M., and Favrin, G. (2007) Structural reorganisation and potential toxicity of oligomeric species formed during the assembly of amyloid fibrils. PLoS Computational Biology 3, 1727-1738.

(4) Ma, B., and Nussinov, R. (2002) Stabilities and conformations of Alzheimer 's Bamyloid peptide oligomers: Sequence effects. Proceedings of the National Academy of Sciences 99, 14126-14131.

(5) Wetzel, R. (2012) Physical chemistry of polyglutamine: Intriguing tales of a monotonous sequence. Journal of Molecular Biology 421, 466-490.

(6) Adegbuyiro, A., Sedighi, F., Pilkington, A. W., Groover, S., and Legleiter, J. (2017) Proteins Containing Expanded Polyglutamine Tracts and Neurodegenerative Disease. Biochemistry 56, 1199-1217.

(7) Gusella, J. F., and MacDonald, M. E. (1995) Huntington's disease. Seminars in Cell Biology 6, 21-28.

(8) Snell, R. G., Macmillan, J. C., Cheadle, J. P., Fenton, I., Lazarou, L. P., Davies, P., Macdonald, M. E., Gusella, J. F., Harper, P. S., and Shaw, D. J. (1993) Relationship between trinucleotide repeat expansion and phenotypic variation in Huntington's disease. Nature Genetics 4, 393-397.

(9) MacDonald, M. E., Ambrose, C. M., Duyao, M. P., Myers, R. H., Lin, C., Srinidhi, L., Barnes, G., Taylor, S. A., James, M., Groot, N., MacFarlane, H., Jenkins, B., Anderson, M. A., Wexler, N. S., Gusella, J. F., Bates, G. P., Baxendale, S., Hummerich, H., Kirby, S., North, M., Youngman, S., Mott, R., Zehetner, G., Sedlacek, Z., Poustka, A., Frischauf, A. M., Lehrach, H., Buckler, A. J., Church, D., Doucette-Stamm, L., O'Donovan, M. C., Riba-Ramirez, L., Shah, M., Stanton, V. P., Strobel, S. A., Draths, K. M., Wales, J. L., Dervan, P., Housman, D. E., Altherr, M., Shiang, R., Thompson, L., Fielder, T., Wasmuth, J. J., Tagle, D., Valdes, J., Elmer, L., Allard, M., Castilla, L., Swaroop, M., Blanchard, K., Collins, F. S., Snell, R., Holloway, T., Gillespie, K., Datson, N., Shaw, D., and Harper, P. S. (1993) A novel gene containing a trinucleotide repeat that is expanded and unstable on Huntington's disease chromosomes. Cell 72, 971-983.

(10) Penney, J. B. J., Vonsattel, J. P., MacDonald, M. E., Gusella, J. F., and Myers, R. H. (1997) CAG repeat number governs the development rate of pathology in Huntington's disease. Annals of neurology 41, 689-692. 
(11) The U.S.-Venezuela Collaborative Research Project Wexler, N. S. (2004) Venezuelan kindreds reveal that genetic and environmental factors modulate Huntington's disease age of onset. Proceedings of the National Academy of Sciences 101, 3498-3503.

(12) Schulte, J., and Littleton, J. T. (2011) The biological function of the Huntingtin protein and its relevance to Huntington's Disease pathology. Current trends in neurology 5, 6578.

(13) von Hörsten, S., Schmitt, I., Nguyen, H. P., Holzmann, C., Schmidt, T., Walther, T., Bader, M., Pabst, R., Kobbe, P., Krotova, J., Stiller, D., Kask, A., Vaarmann, A., RathkeHartlieb, S., Schulz, J. B., Grasshoff, U., Bauer, I., Vieira-Saecker, A. M. M., Paul, M., Jones, L., Lindenberg, K. S., Landwehrmeyer, B., Bauer, A., Li, X. J., and Riess, O. (2003) Transgenic rat model of Huntington's disease. Human Molecular Genetics 12, 617-624.

(14) Yang, S. H., Cheng, P. H., Banta, H., Piotrowska-Nitsche, K., Yang, J. J., Cheng, E. C. H., Snyder, B., Larkin, K., Liu, J., Orkin, J., Fang, Z. H., Smith, Y., Bachevalier, J., Zola, S. M., Li, S. H., Li, X. J., and Chan, A. W. S. (2008) Towards a transgenic model of Huntington's disease in a non-human primate. Nature 453, 921-924.

(15) Hoffher, G., Island, M. L., and Djian, P. (2005) Purification of neuronal inclusions of patients with Huntington's disease reveals a broad range of $\mathrm{N}$-terminal fragments of expanded huntingtin and insoluble polymers. Journal of Neurochemistry 95, 125-136.

(16) Wellington, C. L., Ellerby, L. M., Gutekunst, C. A., Rogers, D., Warby, S., Graham, R. K., Loubser, O., Van Raamsdonk, J., Singaraja, R., Yang, Y. Z., Gafni, J., Bredesen, D., Hersch, S. M., Leavitt, B. R., Roy, S., Nicholson, D. W., and Hayden, M. R. (2002) Caspase cleavage of mutant huntingtin precedes neurodegeneration in Huntington's disease. Journal of Neuroscience 22, 7862-7872.

(17) Miller, J. P., Holcomb, J., Al-Ramahi, I., de Haro, M., Gafni, J., Zhang, N., Kim, E., Sanhueza, M., Torcassi, C., Kwak, S., Botas, J., Hughes, R. E., and Ellerby, L. M. (2010) Matrix metalloproteinases are modifiers of huntingtin proteolysis and toxicity in Huntington's disease. Neuron 67, 199-212.

(18) Cooper, J. K., Schilling, G., Peters, M. F., Herring, W. J., Sharp, A. H., Kaminsky, Z., Masone, J., Khan, F. A., Delanoy, M., Borchelt, D. R., Dawson, V. L., Dawson, T. M., and Ross, C. A. (1998) Truncated N-terminal fragments of huntingtin with expanded glutamine repeats form nuclear and cytoplasmic aggregates in cell culture. Human Molecular Genetics 7, 783-790.

(19) Li, S. H., and Li, X. J. (1998) Aggregation of N-terminal huntingtin is dependent on the length of its glutamine repeats. Human Molecular Genetics 7, 777-782.

(20) Landrum, E., and Wetzel, R. (2014) Biophysical underpinnings of the repeat length dependence of polyglutamine amyloid formation. Journal of Biological Chemistry 289, 10254-10260. 
(21) Kar, K., Jayaraman, M., Sahoo, B., Kodali, R., and Wetzel, R. (2011) Critical nucleus size for disease-related polyglutamine aggregation is repeat-length dependent. Nature structural \& molecular biology 18, 328-336.

(22) Chen, S., Berthelier, V., Yang, W., and Wetzel, R. (2001) Polyglutamine aggregation behavior in vitro supports a recruitment mechanism of cytotoxicity. Journal of Molecular Biology 311, 173-182.

(23) Davies, S. W., Turmaine, M., Cozens, B. A., DiFiglia, M., Sharp, A. H., Ross, C. A., Scherzinger, E., Wanker, E. E., Mangiarini, L., and Bates, G. P. (1997) Formation of neuronal intranuclear inclusions underlies the neurological dysfunction in mice transgenic for the HD mutation. Cell 90, 537-548.

(24) DiFiglia, M., Sapp, E., Chase, K. O., Davies, S. W., Bates, G. P., Vonsattel, J. P., and Aronin, N. (1997) Aggregation of huntingtin in neuronal intranuclear inclusions and dystrophic neurites in brain. Science 277, 1990-1993.

(25) Yang, W. (2002) Aggregated polyglutamine peptides delivered to nuclei are toxic to mammalian cells. Human Molecular Genetics 11, 2905-2917.

(26) Lajoie, P., and Snapp, E. L. (2010) Formation and toxicity of soluble polyglutamine oligomers in living cells. PLOS ONE 5, e15245.

(27) Nucifora, L. G., Burke, K. A., Feng, X., Arbez, N., Zhu, S., Miller, J., Yang, G., Ratovitski, T., Delannoy, M., Muchowski, P. J., Finkbeiner, S., Legleiter, J., Ross, C. A., and Poiriera, M. A. (2012) Identification of novel potentially toxic oligomers formed in vitro from mammalian-derived expanded huntingtin exon-1 protein. Journal of Biological Chemistry 287, 16017-16028.

(28) Drombosky, K. W., Rode, S., Kodali, R., Jacob, T. C., Palladino, M. J., and Wetzel, R. (2018) Mutational analysis implicates the amyloid fibril as the toxic entity in Huntington's disease. Neurobiology of Disease 120, 126-138.

(29) Pieri, L., Madiona, K., Bousset, L., and Melki, R. (2012) Fibrillar a -Synuclein and Huntingtin Exon 1 Assemblies Are Toxic to the Cells. Biophysj 102, 2894-2905.

(30) Saha, I., Mishra, A., Hartl, F. U., and Baumeister, W. (2017) In Situ Architecture and Cellular Interactions of PolyQ Inclusions Article In Situ Architecture and Cellular Interactions of PolyQ Inclusions. Cell 171, 179-187.

(31) Sathasivam, K., Lane, A., Legleiter, J., Warley, A., Woodman, B., Finkbeiner, S., Paganetti, P., Muchowski, P. J., Wilson, S., and Bates, G. P. (2010) Identical oligomeric and fibrillar structures captured from the brains of R6 / 2 and knock-in mouse models of Huntington 's disease 19, 65-78. 
(32) Nekooki-Machida, Y., Kurosawa, M., Nukina, N., Ito, K., Oda, T., and Tanaka, M. (2009) Distinct conformations of in vitro and in vivo amyloids of huntingtin-exon1 show different cytotoxicity. Proceedings of the National Academy of Sciences of the United States of America 106, 9679-9684.

(33) Jayaraman, M., Kodali, R., Sahoo, B., Thakur, A. K., Mayasundari, A., Mishra, R., Peterson, C. B., and Wetzel, R. (2012) Slow amyloid nucleation via a-helix-rich oligomeric intermediates in short polyglutamine-containing huntingtin fragments. Journal of Molecular Biology 415, 881-899.

(34) Saunders, H. M., and Bottomley, S. P. (2009) Multi-domain misfolding: Understanding the aggregation pathway of polyglutamine proteins. Protein Engineering, Design and Selection 22, 447-451.

(35) Thakur, A. K., Jayaraman, M., Mishra, R., Thakur, M., Chellgren, V. M., Byeon, I.-J. L., Anjum, D. H., Kodali, R., Creamer, T. P., Conway, J. F., Gronenborn, A. M., and Wetzel, R. (2009) Polyglutamine disruption of the huntingtin exon $1 \mathrm{~N}$ terminus triggers a complex aggregation mechanism. Nature structural \& molecular biology 16, 380-9.

(36) Lakhani, V. V., Ding, F., and Dokholyan, N. v. (2010) Polyglutamine induced misfolding of Huntingtin Exon1 is modulated by the flanking sequences. PLOS Computational Biology 6, 39-41.

(37) Burke, K. A., Kauffman, K. J., Umbaugh, C. S., Frey, S. L., and Legleiter, J. (2013) The interaction of polyglutamine peptides with lipid membranes is regulated by flanking sequences associated with huntingtin. Journal of Biological Chemistry 288, 1499315005.

(38) Duennwald, M. L., Jagadish, S., Muchowski, P. J., and Lindquist, S. (2006) Flanking sequences profoundly alter polyglutamine toxicity in yeast. Proceedings of the National Academy of Sciences 103, 11045-11050.

(39) Mishra, R., Jayaraman, M., Roland, B. P., Landrum, E., Fullam, T., Kodali, R., Thakur, A. K., Arduini, I., and Wetzel, R. (2012) Inhibiting the nucleation of amyloid structure in a huntingtin fragment by targeting a-helix-rich oligomeric intermediates. Journal of Molecular Biology 415, 900-917.

(40) Ellisdon, A. M., Thomas, B., and Bottomley, S. P. (2006) The two-stage pathway of ataxin-3 fibrillogenesis involves a polyglutamine-independent step. Journal of Biological Chemistry 281, 16888-16896.

(41) Ellisdon, A. M., Pearce, M. C., and Bottomley, S. P. (2007) Mechanisms of Ataxin-3 Misfolding and Fibril Formation: Kinetic Analysis of a Disease-associated Polyglutamine Protein. Journal of Molecular Biology 368, 595-605. 
(42) Nucifora, L. G., Burke, K. A., Feng, X., Arbez, N., Zhu, S., Miller, J., Yang, G., Ratovitski, T., Delannoy, M., Muchowski, P. J., Finkbeiner, S., Legleiter, J., Ross, C. A., and Poiriera, M. A. (2012) Identification of novel potentially toxic oligomers formed in vitro from mammalian-derived expanded huntingtin exon-1 protein. Journal of Biological Chemistry 287, 16017-16028.

(43) Darnell, G., Orgel, J. P. R. O., Pahl, R., and Meredith, S. C. (2007) Flanking polyproline sequences inhibit beta-sheet structure in polyglutamine segments by inducing PPII-like helix structure. Journal of molecular biology 374, 688-704.

(44) Bulone, D., Masino, L., Thomas, D. J., San Biagio, P. L., and Pastore, A. (2006) The interplay between polyQ and protein context delays aggregation by forming a reservoir of protofibrils. PLOS ONE 1, 1-10.

(45) Ignatova, Z., and Gierasch, L. M. (2006) Extended polyglutamine tracts cause aggregation and structural perturbation of an adjacent $\beta$ barrel protein. Journal of Biological Chemistry 281, 12959-12967.

(46) Michalek, M., Salnikov, E. S., and Bechinger, B. (2013) Structure and Topology of the Huntingtin $1-17$ Membrane Anchor by a Combined Solution and Solid-State NMR Approach. Biophysj 105, 699-710.

(47) Sedighi, F., Adegbuyiro, A., and Legleiter, J. (2020) SUMOylation Prevents Huntingtin Fibrillization and Localization onto Lipid Membranes. ACS Chemical Neuroscience 11, 328-343.

(48) Chaibva, M., Jawahery, S., Pilkington, A. W., Arndt, J. R., Sarver, O., Valentine, S., Matysiak, S., and Legleiter, J. (2016) Acetylation within the First 17 Residues of Huntingtin Exon 1 Alters Aggregation and Lipid Binding. Biophysical Journal 111, 349362.

(49) Bhattacharyya, A., Thakur, A. K., Chellgren, V. M., Thiagarajan, G., Williams, A. D., Chellgren, B. W., Creamer, T. P., and Wetzel, R. (2006) Oligoproline effects on polyglutamine conformation and aggregation. Journal of Molecular Biology 355, 524-535.

(50) Darnell, G. D., Derryberry, J., Kurutz, J. W., and Meredith, S. C. (2009) Mechanism of Cis -Inhibition of PolyQ Fibrillation by PolyP : PPII Oligomers and the Hydrophobic Effect. Biophysj 97, 2295-2305.

(51) Dehay, B., and Bertolotti, A. (2006) Critical role of the proline-rich region in Huntingtin for aggregation and cytotoxicity in yeast. Journal of Biological Chemistry 281, 3560835615.

(52) Kim, M. W., Chelliah, Y., Kim, S. W., Otwinowski, Z., and Bezprozvanny, I. (2009) Secondary Structure of Huntingtin Amino-Terminal Region. Structure 17, 1205-1212. 
(53) Crick, S. L., Ruff, K. M., Garai, K., Frieden, C., and Pappu, R. V. (2013) Unmasking the roles of $\mathrm{N}$ - and $\mathrm{C}$-terminal flanking sequences from exon 1 of huntingtin as modulators of polyglutamine aggregation. Proceedings of the National Academy of Sciences of the United States of America 110, 20075-20080.

(54) Sivanandam, V. N., Jayaraman, M., Hoop, C. L., Kodali, R., Wetzel, R., and van der Wel, P. C. A. (2011) The aggregation-enhancing huntingtin $\mathrm{N}$-terminus is helical in amyloid fibrils. Journal of the American Chemical Society 133, 4558-4566.

(55) Williamson, T. E., Vitalis, A., Crick, S. L., and Pappu, R. V. (2010) Modulation of Polyglutamine Conformations and Dimer Formation by the N-Terminus of Huntingtin. Journal of Molecular Biology 396, 1295-1309.

(56) Vitalis, A., and Pappu, R. V. (2011) Biophysical Chemistry Assessing the contribution of heterogeneous distributions of oligomers to aggregation mechanisms of polyglutamine peptides. Biophysical Chemistry 159, 14-23.

(57) Kelley, N. W., Huang, X., Tam, S., Spiess, C., Frydman, J., and Pande, V. S. (2009) The Predicted Structure of the Headpiece of the Huntingtin Protein and Its Implications on Huntingtin Aggregation. Journal of Molecular Biology 388, 919-927.

(58) Atwal, R. S., Xia, J., Pinchev, D., Taylor, J., Epand, R. M., and Truant, R. (2007) Huntingtin has a membrane association signal that can modulate huntingtin aggregation, nuclear entry and toxicity. Human Molecular Genetics 16, 2600-2615.

(59) Michalek, M., Salnikov, E. S., Werten, S., and Bechinger, B. (2013) Membrane interactions of the amphipathic amino terminus of huntingtin. Biochemistry 52, 847-858.

(60) Jayaraman, M., Mishra, R., Kodali, R., Thakur, A. K., Koharudin, L. M. I., Gronenborn, A. M., and Wetzel, R. (2012) Kinetically competing huntingtin aggregation pathways control amyloid polymorphism and properties. Biochemistry 51, 2706-2716.

(61) Tam, S., Spiess, C., Auyeung, W., Joachimiak, L., Chen, B., Poirier, M. A., and Frydman, J. (2009) The chaperonin TRiC blocks a huntingtin sequence element that promotes the conformational switch to aggregation. Nature Structural \& Molecular Biology $16,1279-1286$.

(62) Monsellier, E., Redeker, V., Ruiz-Arlandis, G., Bousset, L., and Melki, R. (2015) Molecular interaction between the chaperone $\mathrm{Hsc} 70$ and the N-terminal flank of huntingtin exon 1 modulates aggregation. Journal of Biological Chemistry 290, 2560-2576.

(63) Caron, N. S., Desmond, C. R., Xia, J., and Truant, R. (2013) Polyglutamine domain flexibility mediates the proximity between flanking sequences in huntingtin. Proceedings of the National Academy of Sciences of the United States of America 110, 14610-5. 
(64) Pennuto, M., Palazzolo, I., and Poletti, A. (2009) Post-translational modifications of expanded polyglutamine proteins: Impact on neurotoxicity. Human Molecular Genetics.

(65) Aiken, C. T., Steffan, J. S., Guerrero, C. M., Khashwji, H., Lukacsovich, T., Simmons, D., Purcell, J. M., Menhaji, K., Zhu, Y. Z., Green, K., LaFerla, F., Huang, L., Thompson, L. M., and Marsh, J. L. (2009) Phosphorylation of threonine 3: Implications for huntingtin aggregation and neurotoxicity. Journal of Biological Chemistry 284, 29427-29436.

(66) Steffan, J. S., Agrawal, N., Pallos, J., Rockabrand, E., Trotman, L. C., Slepko, N., Illes, K., Lukacsovich, T., Zhu, Y. Z., Cattaneo, E., Pandolfi, P. P., Thompson, L. M., and Marsh, J. L. (2004) SUMO Modification of Huntingtin and Huntington's Disease Pathology. Science 304, 100-104.

(67) Ehrnhoefer, D. E., Sutton, L., and Hayden, M. R. (2011) Small changes, big impact: Posttranslational modifications and function of huntingtin in huntington disease. Neuroscientist 17, 475-492.

(68) Díaz-Hernández, M., Valera, A. G., Morán, M. A., Gómez-Ramos, P., AlvarezCastelao, B., Castaño, J. G., Hernández, F., and Lucas, J. J. (2006) Inhibition of 26S proteasome activity by huntingtin filaments but not inclusion bodies isolated from mouse and human brain. Journal of Neurochemistry 98, 1585-1596.

(69) Thompson, L. M., Aiken, C. T., Kaltenbach, L. S., Agrawal, N., Illes, K., Khoshnan, A., Martinez-Vincente, M., Arrasate, M., O'Rourke, J. G., Khashwji, H., Lukacsovich, T., Zhu, Y. Z., Lau, A. L., Massey, A., Hayden, M. R., Zeitlin, S. O., Finkbeiner, S., Green, K. N., LaFerla, F. M., Bates, G., Huang, L., Patterson, P. H., Lo, D. C., Cuervo, A. M., Marsh, J. L., and Steffan, J. S. (2009) IKK phosphorylates Huntingtin and targets it for degradation by the proteasome and lysosome. Journal of Cell Biology 187, 1083-1099.

(70) Gu, X., Greiner, E. R., Mishra, R., Kodali, R., Osmand, A., Finkbeiner, S., Steffan, J. S., Thompson, L. M., Wetzel, R., and Yang, X. W. (2009) Article Serines 13 and 16 Are Critical Determinants of Full-Length Human Mutant Huntingtin Induced Disease Pathogenesis in HD Mice. Neuron 64, 828-840.

(71) Cong, X., Held, J. M., DeGiacomo, F., Bonner, A., Chen, J. M., Schilling, B., Czerwieniec, G. A., Gibson, B. W., and Ellerby, L. M. (2011) Mass spectrometric identification of novel lysine acetylation sites in Huntingtin. Molecular and Cellular Proteomics 10, 1-13.

(72) DiGiovanni, L. F., Mocle, A. J., Xia, J., and Truant, R. (2016) Huntingtin N17 domain is a reactive oxygen species sensor regulating huntingtin phosphorylation and localization. Human Molecular Genetics 25, 3937-3945.

(73) Mishra, R., Hoop, C. L., Kodali, R., Sahoo, B., Wel, P. C. A. van der, and Wetzel, R. (2012) Serine Phosphorylation Suppresses Huntingtin Amyloid Accumulation by Altering Protein Aggregation Properties. Journal of Molecular Biology 424, 1-14. 
(74) Elbaum, M. B., and Zondlo, N. J. (2014) OGlcNAcylation and phosphorylation have similar structural effects in $\alpha$-helices: Post-translational modifications as inducible start and stop signals in a-helices, with greater structural effects on threonine modification. Biochemistry 53, 2242-2260.

(75) Kim, G. H., Kim, J. E., Rhie, S. J., and Yoon, S. (2015) The Role of Oxidative Stress in Neurodegenerative Diseases. Experimental Neurobiology 24, 325-340.

(76) Niedzielska, E., Smaga, I., Gawlik, M., Moniczewski, A., Stankowicz, P., Pera, J., and Filip, M. (2016) Oxidative Stress in Neurodegenerative Diseases. Molecular Neurobiology 53, 4094-4125.

(77) Li, J., Wuliji, O., Li, W., Jiang, Z. G., and Ghanbari, H. A. (2013) Oxidative stress and neurodegenerative disorders. International Journal of Molecular Sciences 14, 2443824475.

(78) Mitomi, Y., Nomura, T., Kurosawa, M., Nukina, N., and Furukawa, Y. (2012) Postaggregation oxidation of mutant huntingtin controls the interactions between aggregates. Journal of Biological Chemistry 287, 34764-34775.

(79) Ceccon, A., Tugarinov, V., and Clore, G. M. (2019) TiO 2 Nanoparticles Catalyze Oxidation of Huntingtin Exon 1-Derived Peptides Impeding Aggregation: A Quantitative NMR Study of Binding and Kinetics. Journal of the American Chemical Society 141, 9497.

(80) Ceccon, A., Schmidt, T., Tugarinov, V., Kotler, S. A., Schwieters, C. D., and Clore, G. M. (2018) Interaction of Huntingtin Exon-1 Peptides with Lipid-Based Micellar Nanoparticles Probed by Solution NMR and Q-Band Pulsed EPR. Journal of the American Chemical Society 140, 6199-6202.

(81) Kegel-Gleason, K. B. (2013) Huntingtin interactions with membrane phospholipids: Strategic targets for therapeutic intervention? Journal of Huntington's Disease 2, 239250.

(82) Orr, A. L., Li, S., Wang, C.-E., Li, H., Wang, J., Rong, J., Xu, X., Mastroberardino, P. G., Greenamyre, J. T., and Li, X.-J. (2008) N-Terminal Mutant Huntingtin Associates with Mitochondria and Impairs Mitochondrial Trafficking. Journal of Neuroscience 28, 27832792.

(83) Panov, A. v., Gutekunst, C.-A., Leavitt, B. R., Hayden, M. R., Burke, J. R., Strittmatter, W. J., and Greenamyre, J. T. (2002) Early mitochondrial calcium defects in Huntington's disease are a direct effect of polyglutamines. Nature Neuroscience 5, 731736. 
(84) Ueda, M., Li, S., Itoh, M., Wang, M., Hayakawa, M., Islam, S., Nakagawa, K., Chen, H., and Nakagawa, T. (2016) Biochemical and Biophysical Research Communications Expanded polyglutamine embedded in the endoplasmic reticulum causes membrane distortion and coincides with Bax insertion. Biochemical and Biophysical Research Communications 474, 259-263.

(85) Liu, K. Y., Shyu, Y. C., Barbaro, B. A., Lin, Y. T., Chern, Y., Thompson, L. M., Shen, C. K. J., and Marsh, J. L. (2015) Disruption of the nuclear membrane by perinuclear inclusions of mutant huntingtin causes cell-cycle re-entry and striatal cell death in mouse and cell models of Huntington's disease. Human Molecular Genetics 24, 1602-1616.

(86) Reddy, P. H., and Shirendeb, U. P. (2012) Mutant huntingtin, abnormal mitochondrial dynamics, defective axonal transport of mitochondria, and selective synaptic degeneration in Huntington's disease. Biochimica et Biophysica Acta - Molecular Basis of Disease 1822, 101-110.

(87) Shirendeb, U. P., Calkins, M. J., Manczak, M., Anekonda, V., Dufour, B., McBride, J. L., Mao, P., and Reddy, P. H. (2012) Mutant Huntingtin's interaction with mitochondrial protein Drp1 impairs mitochondrial biogenesis and causes defective axonal transport and synaptic degeneration in Huntington's disease. Human Molecular Genetics 21, 406-420.

(88) Trettel, F., Rigamonti, D., Hilditch-Maguire, P., Wheeler, V. C., Sharp, A. H., Persichetti, F., Cattaneo, E., and MacDonald, M. E. (2000) Dominant phenotypes produced by the HD mutation in STHdh(Q111) striatal cells. Human Molecular Genetics 9, 2799-2809.

(89) Chang, D. T. W., Rintoul, G. L., Pandipati, S., and Reynolds, I. J. (2006) Mutant huntingtin aggregates impair mitochondrial movement and trafficking in cortical neurons. Neurobiology of Disease 22, 388-400.

(90) Choo, Y. S., Johnson, G. V. W., MacDonald, M., Detloff, P. J., and Lesort, M. (2004) Mutant huntingtin directly increases susceptibility of mitochondria to the calcium-induced permeability transition and cytochrome c release. Human Molecular Genetics 13, 14071420.

(91) del Toro, D., Alberch, J., Lázaro-Diéguez, F., Martín-lbáñ Ez, R., Xifró, X., Egea, G., and Canals, J. M. (2009) Mutant Huntingtin Impairs Post-Golgi Trafficking to Lysosomes by Delocalizing Optineurin/Rab8 Complex from the Golgi Apparatus. Molecular Biology of the Cell 20, 1478-1492.

(92) Qin, Z. H., Wang, Y., Sapp, E., Cuiffo, B., Wanker, E., Hayden, M. R., Kegel, K. B., Aronin, N., and DiFiglia, M. (2004) Huntingtin Bodies Sequester Vesicle-Associated Proteins by a Polyproline-Dependent Interaction. Journal of Neuroscience 24, 269-281.

(93) Kegel, K. B., Kim, M., Sapp, E., Mclntyre, C., Castano, J. G., Aronin, N., and DiFiglia, M. (2000) Huntingtin expression stimulates endosomal-lysosomal activity, endosome tubulation, and autophagy. Journal of Neuroscience 20, 7268-7278. 
(94) Yu, Z. X., Li, S. H., Evans, J., Pillarisetti, A., Li, H., and Li, X. J. (2003) Mutant huntingtin causes context-dependent neurodegeneration in mice with Huntington's disease. Journal of Neuroscience 23, 2193-2202.

(95) Song, W., Chen, J., Petrilli, A., Liot, G., Klinglmayr, E., Zhou, Y., Poquiz, P., Tjong, J., Pouladi, M. A., Hayden, M. R., Masliah, E., Ellisman, M., Rouiller, I., Schwarzenbacher, R., Bossy, B., Perkins, G., and Bossy-Wetzel, E. (2011) Mutant huntingtin binds the mitochondrial fission GTPase dynamin-related protein-1 and increases its enzymatic activity. Nature medicine 17, 377-82.

(96) Yano, H., Baranov, S. V., Baranova, O. V., Kim, J., Pan, Y., Yablonska, S., Carlisle, D. L., Ferrante, R. J., Kim, A. H., and Friedlander, R. M. (2014) Inhibition of mitochondrial protein import by mutant huntingtin. Nature Neuroscience 17, 822-831.

(97) Ghosh, R., Wood-Kaczmar, A., Dobson, L., Smith, E. J., Sirinathsinghji, E. C., Kriston-Vizi, J., Hargreaves, I. P., Heaton, R., Herrmann, F., Abramov, A. Y., Lam, A. J., Heales, S. J., Ketteler, R., Bates, G. P., Andre, R., and Tabrizi, S. J. (2020) Expression of mutant exon 1 huntingtin fragments in human neural stem cells and neurons causes inclusion formation and mitochondrial dysfunction. FASEB Journal 34, 8139-8154.

(98) Kegel, K. B., Sapp, E., Alexander, J., Valencia, A., Reeves, P., Li, X., Masso, N., Sobin, L., Aronin, N., and DiFiglia, M. (2009) Polyglutamine expansion in huntingtin alters its interaction with phospholipids. Journal of Neurochemistry 110, 1585-1597.

(99) Burke, K. A., Hensal, K. M., Umbaugh, C. S., Chaibva, M., and Legleiter, J. (2013) Huntingtin disrupts lipid bilayers in a polyQ-length dependent manner. Biochimica et Biophysica Acta - Biomembranes 1828, 1953-1961.

(100) Kegel, K. B., Schewkunow, V., Sapp, E., Masso, N., Wanker, E. E., DiFiglia, M., and Goldmann, W. H. (2009) Polyglutamine expansion in huntingtin increases its insertion into lipid bilayers. Biochemical and Biophysical Research Communications 387, 472-475.

(101) Kegel, K. B., Sapp, E., Alexander, J., Valencia, A., Reeves, P., Li, X., Masso, N., Sobin, L., Aronin, N., and DiFiglia, M. (2009) Polyglutamine expansion in huntingtin alters its interaction with phospholipids. Journal of Neurochemistry 110, 1585-1597.

(102) Nagarajan, A., Jawahery, S., and Matysiak, S. (2014) The effects of flanking sequences in the interaction of polyglutamine peptides with a membrane bilayer. Journal of Physical Chemistry B 118, 6368-6379.

(103) Tao, M., Pandey, N. K., Barnes, R., Han, S., and Langen, R. (2019) Structure of Membrane-Bound Huntingtin Exon 1 Reveals Membrane Interaction and Aggregation Mechanisms. Structure 27, 1570-1580.e4.

(104) Pandey, N. K., Isas, J. M., Rawat, A., Lee, R. v., Langen, J., Pandey, P., and Langen, R. (2017) The 17-residue-long $N$ terminus in huntingtin controls step-wise aggregation in solution and on membranes via different mechanisms. Journal of Biological Chemistry 293, 2597-2605. 
(105) Beasley, M., Groover, S., Valentine, S. J., and Legleiter, J. (2021) Lipid headgroups alter huntingtin aggregation on membranes. Biochimica et Biophysica Acta Biomembranes 1863, 183497.

(106) Chaibva, M., Gao, X., Jain, P., Campbell, W. A., Frey, S. L., and Legleiter, J. (2017) Sphingomyelin and GM1 Influence Huntingtin Binding to, Disruption of, and Aggregation on Lipid Membranes. ACS Omega 3, 273-285.

(107) Gao, X., Campbell, W. A., Chaibva, M., Jain, P., Leslie, A. E., Frey, S. L., and Legleiter, J. (2016) Cholesterol Modifies Huntingtin Binding to, Disruption of, and Aggregation on Lipid Membranes. Biochemistry 55, 92-102. 


\section{Mitochondrial membranes modify mutant huntingtin aggregation}

Huntington's disease (HD) is a neurodegenerative disease caused by the expansion of a polyglutamine (polyQ) tract near the $\mathrm{N}$-terminus of the huntingtin (htt) protein. Expanded polyQ tracts are prone to aggregate into oligomers and insoluble fibrils. Mutant htt (mhtt) localizes to variety of organelles, including mitochondria. Specifically, mitochondrial defects, morphological alteration, and dysfunction are observed in HD. Mitochondrial lipids, cardiolipin (CL) in particular, are essential in mitochondria function and have the potential to directly interact with htt, altering its aggregation. Here, the impact of mitochondrial membranes on htt aggregation was investigated using a combination of mitochondrial membrane mimics and tissue-derived mitochondrial-enriched fractions. The impact of exposure of outer and inner mitochondrial membrane mimics (OMM and IMM respectively) to mhtt was explored. OMM and IMM reduced mhtt fibrillization, with IMM having a larger effect. The role of $\mathrm{CL}$ in mhtt aggregation was investigated using a simple PC system with varying molar ratios of $\mathrm{CL}$. Lower molar ratios of $\mathrm{CL}(<5 \%)$ promoted fibrillization; however, increased CL content retarded fibrillization. As revealed by in situ AFM, mhtt aggregation and associated membrane morphological changes at the surface of OMM mimics was markedly different compared to IMM mimics. While globular deposits of mhtt with few fibrillar aggregates were observed on OMM, plateaulike domains were observed on IMM. A similar impact on htt aggregation was observed with exposure to purified mitochondrial-enriched fractions. Collectively, these observations suggest mitochondrial membranes heavily influence htt aggregation with implication for HD. 


\section{Introduction: Mutant htt aggregates, localizes to organelles and promotes organelle disruption}

Huntington's Disease (HD) is a progressive neurological disease caused by the expansion of a polyglutamine (polyQ) track in the huntingtin protein (htt). ${ }^{1}$ PolyQ expansions beyond a critical threshold ( $35 \mathrm{Q})$ promotes htt to aggregate into oligomers and amyloid fibrils that eventually agglomerate to form inclusions within cells. ${ }^{2}$ The extent of aggregation, ${ }^{3,4}$ and toxicity ${ }^{5,6}$ is strongly correlated with polyQ length. The precise toxic aggregate entities remain elusive. Rather than inclusions, many studies show that the diffuse htt fraction correlates with cell death. ${ }^{4,5,7}$ However, this diffuse fraction contains a complex mixture of monomers, oligomers ${ }^{8,9}$ and fibrils. ${ }^{10}$ In addition, monomers, ${ }^{11}$ oligomers ${ }^{12}$ and fibrils ${ }^{13}$ all demonstrate toxic gain of function.

While expanded polyQ is central to htt aggregation, other factors, including peptide sequences flanking poly $\mathrm{Q}^{14,15}$ and membranous surfaces, ${ }^{16}$ influence aggregation. The first 17 amino acids (Nt17) of htt that directly precede polyQ enhances aggregation by facilitating the formation of $\alpha$-helix-rich oligomer intermediates. ${ }^{15,17} \mathrm{Nt} 17$ functions as a lipid binding domain by forming an amphipathic $\alpha$-helix, ${ }^{18,19}$ and interactions between Nt17 and lipids can promote distinct aggregation mechanisms. ${ }^{20}$ The ability of Nt17 to bind membranes has been linked to $\mathrm{htt}$ trafficking. ${ }^{21,22} \mathrm{Htt}$ is significantly associated with membranous organelles, including mitochondria, endoplasmic reticulum (ER), the nuclear envelope, tubulovesicles, endosomes, lysosomes, and synaptic vesicles. ${ }^{23-28} \mathrm{Htt}$ aggregates and inclusions accumulate brain lipids in mouse models. ${ }^{29-31} \mathrm{Htt}$ aggregation can damage lipid membranes ${ }^{32-35}$ and disrupt organelle integrity. ${ }^{27,28,36,37}$ Beyond being 
a target of aggregated htt, organelles provide subcellular environments that facilitate aggregation by localizing and inducing structural changes in htt. ${ }^{38}$

A membranous organelle impacted by htt aggregates are the mitochondria. Wild type htt and mutant htt (mhtt) fragments localize to mitochondria. ${ }^{24}$ However, mhtt detrimentally impacts mitochondria trafficking, ${ }^{24}$ alters protein import, ${ }^{39}$ and bind other mitochondrial proteins to promote mitophagy. ${ }^{40}$ Mitochondrial dysfunction in HD is further demonstrated by increased lactate in the cortex and basal ganglia ${ }^{41}$ and decreased activities of complexes II and III of the electron-transport chain. ${ }^{42}$ Lymphoblast mitochondria from HD patients and brain mitochondria from YAC transgenic mice expressing mutant htt displayed lower membrane potentials and depolarized at lower calcium loads. ${ }^{25}$ Importantly, htt directly interacts with mitochondria, as demonstrated by subfractionation of mitochondria from knock-in mouse models. ${ }^{43}$ Specifically, mutant htt associated with the outer mitochondrial membrane (OMM), increasing sensitivity to calcium-induced permeabilization, i.e. membrane damage ${ }^{43} \mathrm{mhtt}$ also localizes to the intermembrane where it is bound to an inner mitochondrial membrane (IMM) protein, eliciting proteome imbalance. ${ }^{44}$ In addition, oligomers of htt are associated with mitochondrial structural proteins, such as dynamin related protein (DRP1), ${ }^{45-47}$ and lead to mitochondrial fragmentation, abnormal mitochondrial dynamics, and oxidative DNA damage. ${ }^{26,45,48}$

Despite mitochondrial dysfunction being established in HD, little is known concerning how the presence of mitochondrial surfaces influence htt aggregation. Here, the aggregation of htt-exon1 with 46 glutamine repeats (htt-exon1(46Q)) was evaluated in the presence of purified mitochondria, which suppressed fibril formation and altered 
oligomer morphology. To distinguish the role of the $\mathrm{OMM}$ and IMM, the aggregation and membrane activity of htt-exon1(46Q) in the presence of vesicles that mimicked the composition of these membranes was investigated. From these studies, a prominent role for cardiolipin (CL) was revealed.

\subsection{Methods}

2.2.1 Purification of GST-htt exon1 fusion protein: Gluthathione S-transferase (GST)-htt-exon1(46Q) fusion proteins was expressed in Escherichia coli after induction with isopropyl thiogalactopyranoside (IPTG) for $4 \mathrm{~h}$ at $30^{\circ} \mathrm{C}$ and purified as previously described. ${ }^{49}$ Briefly, after expression, the cells were lysed by a combination of lysozyme and probe sonication. The resulting mixture was purified using affinity liquid chromatography (Bio-Rad LPLC, GST-affinity column). Relevant protein fractions and purity were determined by SDS-PAGE. Prior to every experiment, GST-htt-exon1(46Q) fusion proteins were subjected to high spin centrifugation $\left(22,000 \times \mathrm{g}, 4^{\circ} \mathrm{C}, 30 \mathrm{~min}\right)$ to remove pre-existing aggregates, and protein concentration was measured with a Bradford assay. Cleavage of GST was facilitated by incubation with factor Xa (New England Biolabs). All experiments were carried out in an aggregation buffer $[50 \mathrm{mM}$ Tris- $\mathrm{HCl}(\mathrm{pH}$ 7) and $150 \mathrm{mM} \mathrm{NaCl}$.

2.2.2 Isolation of Mitochondrial-Enriched Fractions: Mitochondria-enriched fractions (MEFs) were isolated separately from bovine liver or murine brain using a Mitochondria Isolation Kit for Tissue (ThermoFisher, Catalog \# 89801). In short, tissues were cut to sizes less than $200 \mathrm{mg}$, washed with PBS before being homogenized with a dismembrator. The homogenized tissue was centrifuged $\left(1000 \times \mathrm{g}, 3 \mathrm{~min}, 4^{\circ} \mathrm{C}\right)$, the supernatant discarded, and the pellet retained. Then, the pellet was resuspended in 
BSA/Mitochondria Isolation reagent $A$. The suspension was vortexed for $5 \mathrm{~s}$ and allowed to rest on ice for 2 min before the addition of Mitochondria Isolation reagent B. Prior to addition of Mitochondria isolation reagent $C$, the suspension was periodically agitated and centrifuged at high speed for $5 \mathrm{~min}$. To remove the supernatant, mitochondria were pelleted by centrifugation $\left(700 \times \mathrm{g}, 10 \mathrm{~min}, 4^{\circ} \mathrm{C}\right)$. The pellet was washed, centrifuged at $12000 \times \mathrm{g}$ to remove residual supernatant before being stored at $-80{ }^{\circ} \mathrm{C}$ until use. Enrichment of mitochondria was evaluated by an ELISA assay for cytochrome C (Cytochrome C ELISA Kit, Abcam) and Western blot analysis. Prior to each experiment, mitochondria pellets were thawed on ice, and the mitochondria concentration was determined by measuring total protein content using a Bradford Assay.

2.2.3 Preparation of Lipid Vesicles: All lipids (PC, PE, PI, PS and CL) were purchased from Avanti Polar Lipids (Alabaster, $\mathrm{AL}$ ) and stored at $-20{ }^{\circ} \mathrm{C}$ until use. Respectively, these lipids were dissolved in chloroform and mixed to achieve appropriate molar ratios (53:27:9:7:4 for OMM, ${ }^{50,51} 45: 25: 9: 6: 15$ for $\mathrm{IMM}^{50}$ and a third mimic, IMM $\mathrm{CL}$, which was made by maintaining the molar ratio of IMM but without CL. In addition, mixtures with the following CL:PC molar ratios: (1) 0:100, (2) 3: 97 (3) 5: 95 (4) 11:89 and (5) 18:82 were made. Chloroform was removed using an Eppendorf Vacufuge. All dried lipid films were reconstituted to $1 \mathrm{mg} / \mathrm{ml}$ in the aggregation buffer, subjected to 10 freeze/thaw cycles, and bath sonicated for 30 mins to obtain lipid vesicles.

2.2.4 Thioflavin-T Fluorescence Assay: Htt-exon1(46Q), lipid vesicles and thioflavin T (ThT, Sigma-Aldrich, St. Louis, MO) were adjusted to a final concentration of $20 \mu \mathrm{M}, 0.15 \mathrm{mg} / \mathrm{ml}$ and $0.05 \mathrm{mg} / \mathrm{ml}$ respectively. Using a SpectraMax M2 and black Costar 96-well clear flat bottom plates set to $37^{\circ} \mathrm{C}$, ThT fluorescence kinetics was monitored for 
$18 \mathrm{~h}$ at $440 \mathrm{~nm}$ excitation and $484 \mathrm{~nm}$ emission. Fibril disaggregation ThT experiments were performed by mixing preformed fibrils ( $30 \mu \mathrm{M}, 8 \mathrm{~h})$ with neat buffer, lipid vesicles, and ThT to achieve a final htt-exon1 concentrations of $10 \mu \mathrm{M}$ (fibrils), and ThT concentration of $0.05 \mathrm{mg} / \mathrm{ml}$. Lipid vesicles final concentrations were varied between 7.5 $\mathrm{mg} / \mathrm{ml}$ and $22.5 \mathrm{mg} / \mathrm{ml}$ to achieved protein to lipid concentrations of 1:10, 1:20 and 1:30. Experiments were carried out at $37^{\circ} \mathrm{C}$ and monitored for $24 \mathrm{~h}$.

2.2.5 Atomic Force Microscopy (AFM): AFM experiments were performed with a Nanoscope V Multimode atomic force microscope (Veeco, Santa Barbara, CA) equipped with a closed-loop vertical engage J-scanner. For ex situ AFM, incubations of httexon1 $(46 \mathrm{Q})(20 \mu \mathrm{M})$ with and without the various lipid vesicles $(0.15 \mathrm{mg} / \mathrm{ml}$ or $\sim 200 \mu \mathrm{M})$ or MEFs (1.4 mg/ml BSA equivalent) were maintained at $37^{\circ} \mathrm{C}$. Aliquots of the incubations drawn at various time points were deposited on freshly cleaved mica (Ted Pella Inc., Redding, CA) and allowed to rest for $75 \mathrm{~s}$. The mica was then washed with $200 \mu \mathrm{L}$ of ultrapure water and dried with a gentle stream of air. Dried samples were measured by ex situ AFM with oxide-sharpened silicon cantilever with a nominal spring constant of 40 $\mathrm{N} / \mathrm{m}$ and resonance frequency of $\sim 300 \mathrm{kHz}$.

For in situ AFM experiments, a fluid cell equipped with a silicon nitride cantilever with a $0.1 \mathrm{~N} / \mathrm{m}$ nominal spring constant was used. The drive frequency ranged between 7.5-9.5 kHz, and scan rate was maintained between 1.5 - $2 \mathrm{~Hz}$. Initially, background scans of freshly cleaved mica under neat buffer $(20 \mu \mathrm{L})$ were taken to ensure the cleanliness of the fluid cell. Next, an equal volume of the prepared OMM or IMM vesicles were introduced to a final concentration of $0.5 \mathrm{mg} / \mathrm{ml}$ within the fluid cell. Bilayer formation on mica via vesicle fusion was observed by continuous imaging. Once sufficient bilayer 
formed, htt-exon1(46Q) was injected to achieve a final protein concentration of $10 \mu \mathrm{M}$ and lipid concentration of $0.25 \mathrm{mg} / \mathrm{ml}$ in the fluid cell. After protein injection, images were collected continuously for $60-120$ min. For both ex situ and in situ AFM experiments, images were processed and analyzed with Matlab equipped with the image processing toolbox (MathWorks, Natick, MA) as previously described. ${ }^{52}$

2.2.6 Filter Retardation and Dot Blot Assay: Incubations of Isolated mitochondria (1.4 mg/ml BSA equivalent) with and without $20 \mu \mathrm{M}$ htt exon1(46Q) were maintained at $37^{\circ} \mathrm{C}$. After $1,3,5$ and $8 \mathrm{~h}$ of incubation, $3 \mu \mathrm{L}$ aliquots were drawn from each incubation and boiled in $610 \mu \mathrm{L}$ of $0.5 \%$ sodium dodecyl sulfate (SDS) loading buffer for $5 \mathrm{~min}$. Boiled samples were cooled to room temperature and serially diluted with Tris Buffered saline (TBS, $25 \mathrm{mM}$ Tris) to achieve successive halved concentrations of $1.2 \mu \mathrm{g}, 0.6 \mu \mathrm{g}$ and 0.3 $\mu \mathrm{g}$ of htt exon1(46Q). Similar volumes were drawn from the test sample (htt + mitochondria) and negative control. A slot blot manifold (GE Healthcare) with pre-wet nitrocellulose membrane (NCM, $0.45 \mu \mathrm{m}$ pore size, GE Healthcare) was assembled and connected to vacuum. $300 \mu \mathrm{L}$ of each serial dilution was loaded into the slots of the manifold. After all loaded sample were pulled through the membrane by vacuum, the membrane was carefully removed, dried at room temperature, and soaked overnight in blocking buffer ( $5 \%$ milk solution in TBS) at $4{ }^{\circ} \mathrm{C}$. Blocked membranes were incubated on an orbital shaker for $1 \mathrm{~h}$ with htt-specific monoclonal primary antibody, MW8 (specific for the C-terminal stretch of htt-exon1, detects fibrils, Developmental Studies Hybridoma Bank, University of lowa, 1:350 dilution) or with voltage-dependent anion channel (VDAC) Polyclonal Antibody (Thermo Fisher Scientific, catalog \# PA1-954A, RRID AB_2304154). After washing with TBS, membranes were incubated on an orbital shaker for $1 \mathrm{~h}$ with the 
secondary Goat anti-mouse or Goat anti-rabbit alkaline phosphatase antibody (1:1000 dilution). For color development, the membrane was dried at room temperature and incubated in BCIP/NBT substrate for $10 \mathrm{~min}$. Dot blots were performed by depositing aliquots of samples after $0,1,3,5$, and $8 \mathrm{~h}$ of incubation directly onto NCM. Membranes were blocked in milk solution before incubation in soluble huntingtin specific monoclonal primary antibody, MW1 (specific for the polyQ stretch, detects monomeric htt, Developmental Studies Hybridoma Bank, University of lowa, 1:350 dilution). The membrane was then incubated in secondary antibody and developed as described for filter retardation assay.

For disaggregation experiments, htt-exon1(46Q) was incubated at $30 \mu \mathrm{M}$ for $8 \mathrm{~h}$. Incubations of htt-exon1(46Q) was mixed with neat buffer and with and without mitochondrial enriched fractions (final concentration $1.4 \mathrm{mg} / \mathrm{ml}$ ) or with and without lipid vesicles (final concentration $0.15 \mathrm{mg} / \mathrm{ml}$, or htt:lipid 1:10). Incubations were maintained at $37^{\circ} \mathrm{C}$ for $8 \mathrm{~h}$. Aliquots were taken after 1 and $8 \mathrm{~h}$ and boiled in SDS, deposited on NCM probed with MW8 and developed as described above.

2.2.7 Monomer loss assays: Incubations of MEFs (1.4mg/ml BSA equivalent) with and without $20 \mu \mathrm{M}$ htt-exon1(46Q) were incubated at $37^{\circ} \mathrm{C}$. In addition, an incubation of $20 \mu \mathrm{M}$ htt-exon1(46Q) without MEFs was used as a control. Aliquots were taken from these incubations at various time points $(0,1,3$, and $5 \mathrm{~h})$ and centrifuged at $20000 \times \mathrm{g}$ for 40 min. The concentration of the resulting supernatant was determined with a Bradford assay performed on a NanoDrop spectrophotometer (Thermo Fisher Scientific). To determine the concentration of mitochondria-bound htt, concentration of incubations of htt and mitochondria were corrected for mitochondrial backgrounds of control 
experiments. Supernatants were also evaluated with a dot blot assay performed as described above.

\subsection{Results}

2.3.1 Mitochondrial membrane mimics reduce htt fibril formation. All experiments in this study were performed with a mutant htt fragment that expresses exon1 with $46 \mathrm{Q}$ (htt-exon1(46Q)), which was purified from Escherichia coli as a soluble fusion with glutathione S-transferase (GST). After purification, aggregation is initiated by addition of factor Xa, which cleaves GST. With the knowledge that lipids influence htt aggregation,,$^{20,34,35,53}$ it is plausible that the distinct membranes of mitochondria influence aggregation. The OMM and IMM are mostly composed of similar lipids but of varying

$=$ OMM $(100 \mu \mathrm{M})=$ OMM $(200 \mu \mathrm{M})$
$=$ OMM $(300 \mu \mathrm{M})=$ ThT background

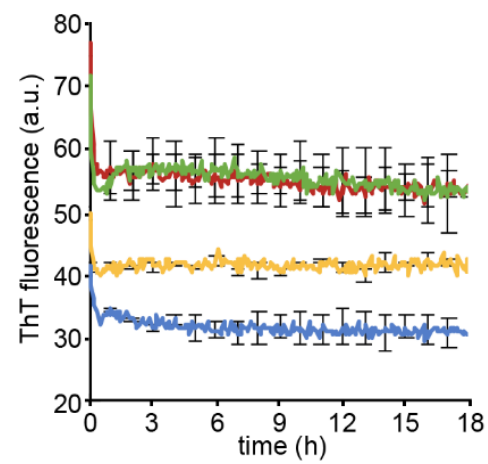

$$
\begin{aligned}
& - \text { IMM }(100 \mu \mathrm{M})=\text { IMM }(200 \mu \mathrm{M}) \\
& - \text { IMM }(300 \mu \mathrm{M})=\text { ThT background }
\end{aligned}
$$
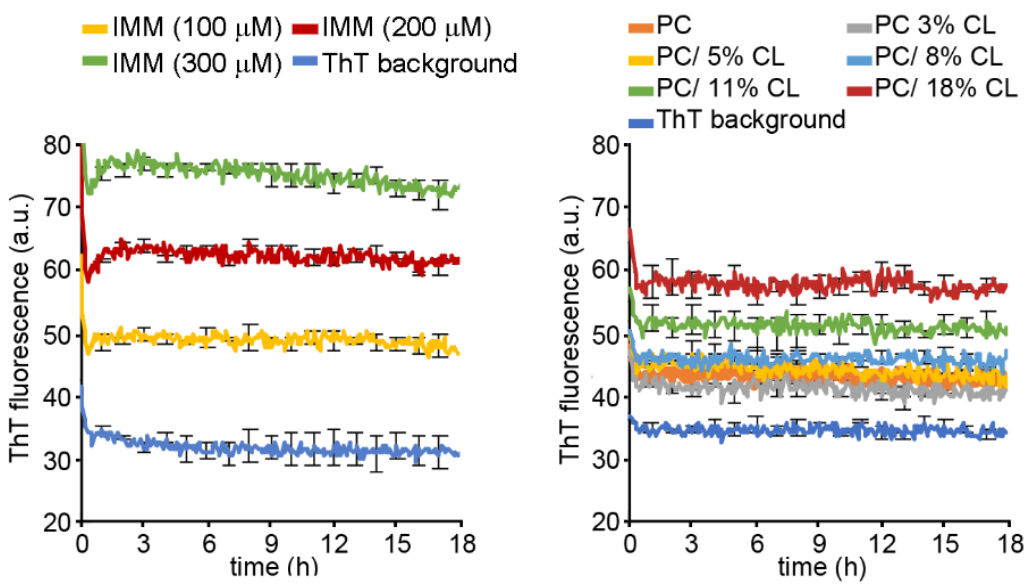

Figure 2.1. Raw ThT signals associated with the different lipid vesicles used to study htt aggregation reported in Figures 2.2A, 2.3 and 2.5A. Error bars represent SEM and are provided every $0.5 \mathrm{~h}$.

abundance. Therefore, two distinct lipid vesicles systems were used to mimic the OMM (PC:53\%, PE:27\%, PI:9\%, PS:7\%, CL:4\%) $)^{50,51}$ and IMM (PC:45\%, PE:25\%, PI:9\%, PS:6\%, CL:15\%) ${ }^{50}$ to determine their impact on htt-exon1(46Q) aggregation. Htt- 
exon1 (46Q) was incubated $\left(20 \mu \mathrm{M}, 37^{\circ} \mathrm{C}\right)$ alone and with mitochondria mimics (OMM and IMM) at a molar protein to lipid ratio of 1:10. Aggregation was monitored using a ThT
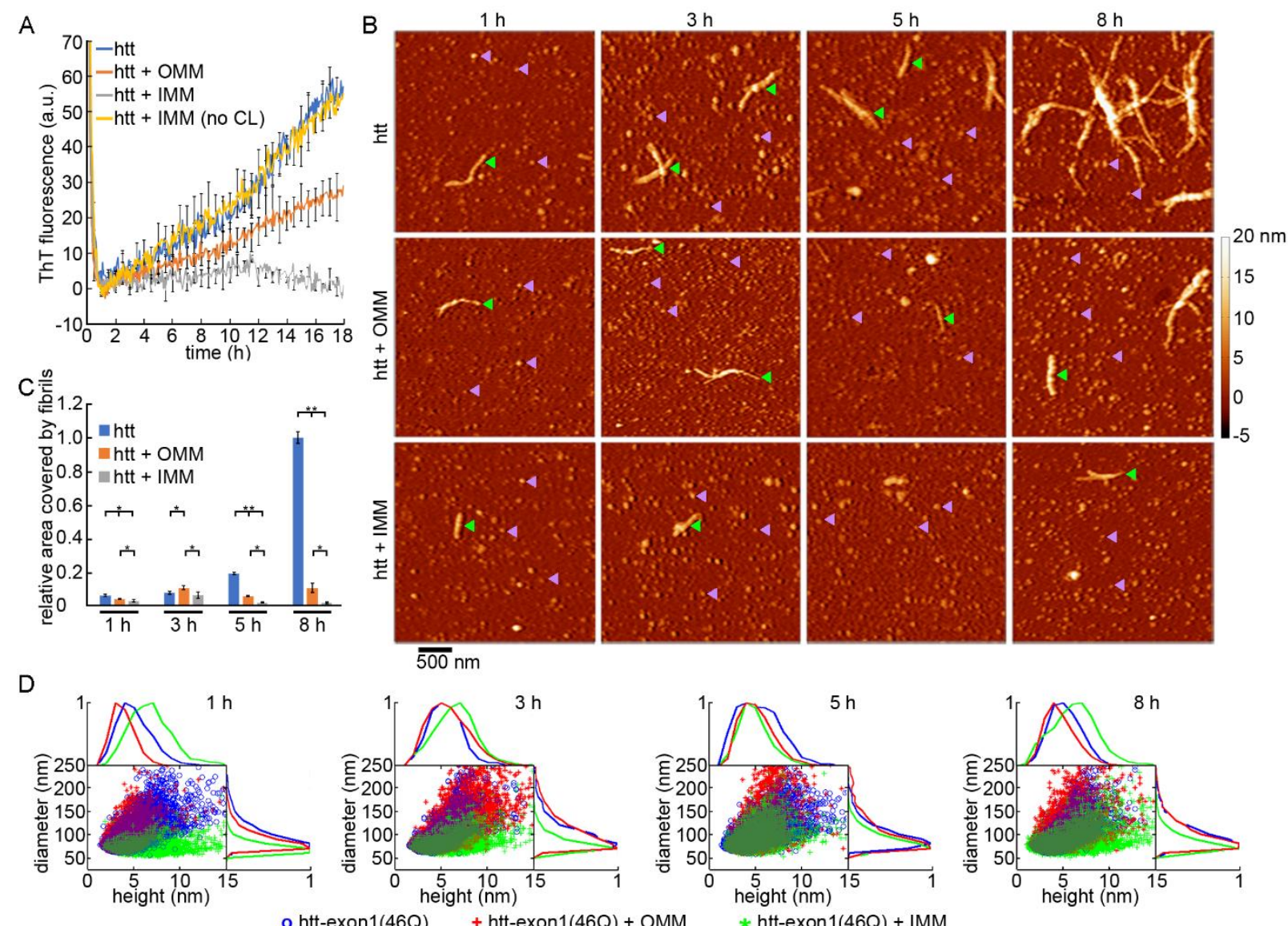

Figure 2.2. Mitochondrial membrane mimics inhibit fibril formation. (A) ThT analysis of the aggregation of htt-exon1(46Q) in the presence or absence of mitochondrial membrane mimics OMM, IMM, and IMM without CL (lipid to protein molar ratio of 10:1). Error bars represent SEM and are provided every $0.5 \mathrm{~h}$ (B) Representative AFM Images of htt exon 1(46Q) incubated with and without IMM and OMM. (C) Analysis of the surface area covered by fibrils as a function of time. Error bars represent SEM. * represents $p$ $<0.05$ and ${ }^{* *}$ represents $p<0.01$. (D) Correlation plots of average diameter vs height of all oligomeric aggregates of htt exon1(46Q) (blue), htt exon1(46Q) + OMM (red), and httexon1(46Q) + IMM (green). For each correlation plot, the height (top) and diameter (right) histograms are provided.

assay, which fluoresces when bound to $\beta$-sheet rich fibrils. While the raw signal shifts with OMM and IMM vesicles without htt, the ThT fluorescent background associated with vesicles is stable (Fig. 2.1), demonstrating that the addition of lipid does not interfere with 
the assay. Both mitochondria membrane mimics reduced fibrillization with IMM having the larger impact (Fig. 2.2A). The impact of OMM and IMM on aggregation was dependent on the lipid to protein ratio, as increased lipid concentration inhibited fibrillization more strongly (Fig. 2.3). Although the mimics are similar in lipids composition, CL content was the largest variable with IMM having significantly more CL. Therefore, additional ThT assays were performed with the addition of vesicles comprised of the same ratios of lipids as IMM but without any CL. With CL excluded from the lipid vesicles, the inhibitory effect of the mimics was lost (Fig. 2.2A).
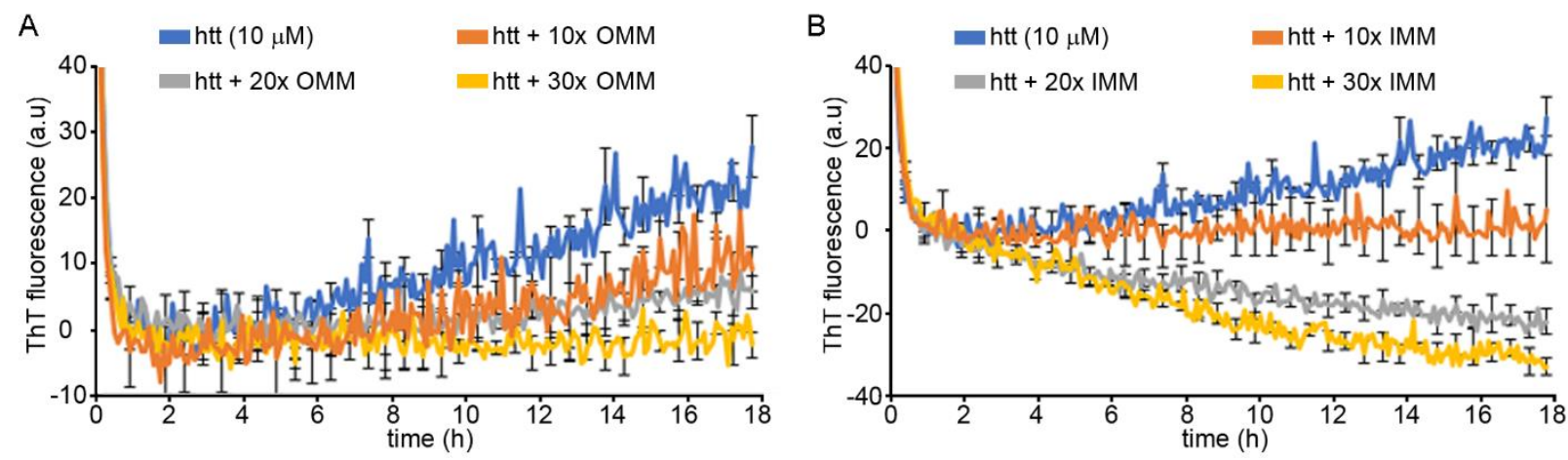

Figure 2.3. Dose dependent impact of (A) OMM and (B) IMM vesicles on httexon1(46Q) (10 $\mu \mathrm{M})$ aggregation as assessed by ThT assays. Error bars represent SEM and are provided every $0.5 \mathrm{~h}$.

To further evaluate the impact of mitochondrial membranes on htt aggregation, httexon1(46Q) was incubated $\left(37^{\circ} \mathrm{C}\right)$ at $20 \mu \mathrm{M}$ in the absence or presence of OMM or IMM mimicking vesicles at a molar peptide to lipid ratio of 1:10. These incubations were sampled at $1,3,5$, and $8 \mathrm{~h}$ for AFM analysis. Control incubations of lipid vesicles alone were also deposited on mica to determine lipid backgrounds (Fig. 2.4). The extent of fibril formation in the presence of the different lipid vesicle systems was evaluated by AFM 
image analysis using custom scripts written in Matlab. These scripts identify individual aggregates in images and measure a variety of morphological features. To distinguish fibrils from oligomers, it was determined that fibrils and fibril bundles occupied more than $9,000 \mathrm{~nm}^{2}$ on the mica surface. Using that criteria as a filter, the relative area of the surface covered by fibrils was determined for all conditions. The analysis was normalized to the maximum fibril content associated with the htt-exon1(46Q) control at $8 \mathrm{~h}$. Within 1 h, htt-exon1(46Q) fibrils were observed in the control, and the number and size of fibrils increased with time (Fig. 2.2B-C). By 5 and $8 \mathrm{~h}$, larger fibrils with entangled morphologies were present.
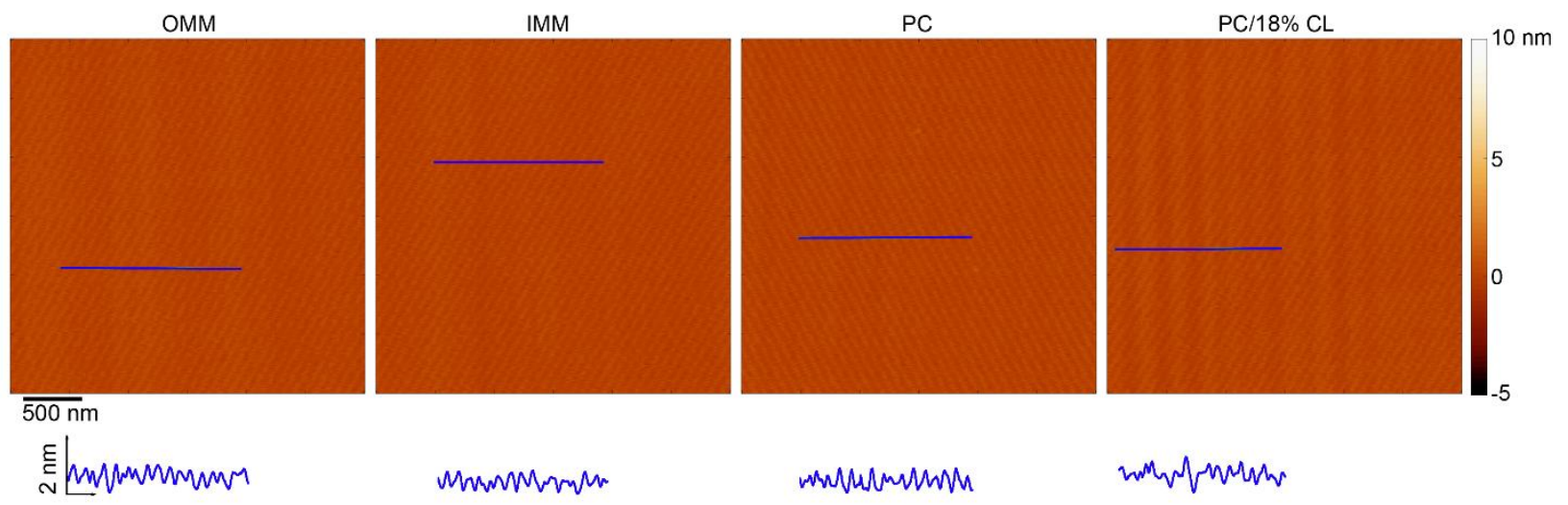

Figure 2.4. Control ex situ AFM images of backgrounds associated with the lipid systems deposited on mica and used to study htt aggregation. The blue lines correspond to the height profiles directly below each image.

While small fibrils formed within $1 \mathrm{~h}$ in the presence of mitochondrial membrane mimics, both IMM and OMM significantly reduced fibril coverage (Fig. 2.2B-C). Consistent with the ThT assay, the inhibitory effect of IMM was larger compared with OMM.

Next, htt oligomer morphology formed in the presence of OMM and IMM mimics was analyzed. For this, oligomers were defined as being at least $1.0 \mathrm{~nm}$ in height with an 
occupied area of 500 to $9,000 \mathrm{~nm}^{2}$ and an aspect ratio (longest distance across to shortest distance across) of less than 3.0, indicating a globular structure. Htt-exon1(46Q) oligomers formed in the presence of $\mathrm{OMM}$ vesicles were morphologically similar to oligomers formed in the absence of lipid (Fig.2.2D). That is, there was substantial overlap in correlation plot of diameter vs height and histograms. Oligomer formed in the absence and presence of OMM vesicles typically displayed mode heights of $\sim 4.5-6 \mathrm{~nm}$ at each time point (Fig. 2.2D). However, htt oligomers formed in the presence of IMM vesicles were initially distinguishable from control after $1 \mathrm{~h}$ of incubation as a significant population of taller aggregates was observed (mode height of $\sim 6-9 \mathrm{~nm}$ ). These taller oligomers persisted after $3 \mathrm{~h}$ of incubation, but were less pronounced at later time points. At both the 1 and $3 \mathrm{~h}$ time points, the height distribution of htt oligomers formed in the presence of IMM were significantly different $(p<0.05)$ to those observed for htt incubated alone and with OMM. Due to the larger heights of oligomers formed in the presence of IMM vesicles at 1 and $3 \mathrm{~h}$, these appear as a distinct population across the bottom of the correlation plot.

2.3.2 Cardiolipin impacts htt aggregation. As changes in htt aggregation in the presence of mitochondrial membrane mimics correlated with $\mathrm{CL}$ content, a simplified system was used to further clarify the role of CL. Using a ThT assay, htt-exon1(46Q) aggregation was 

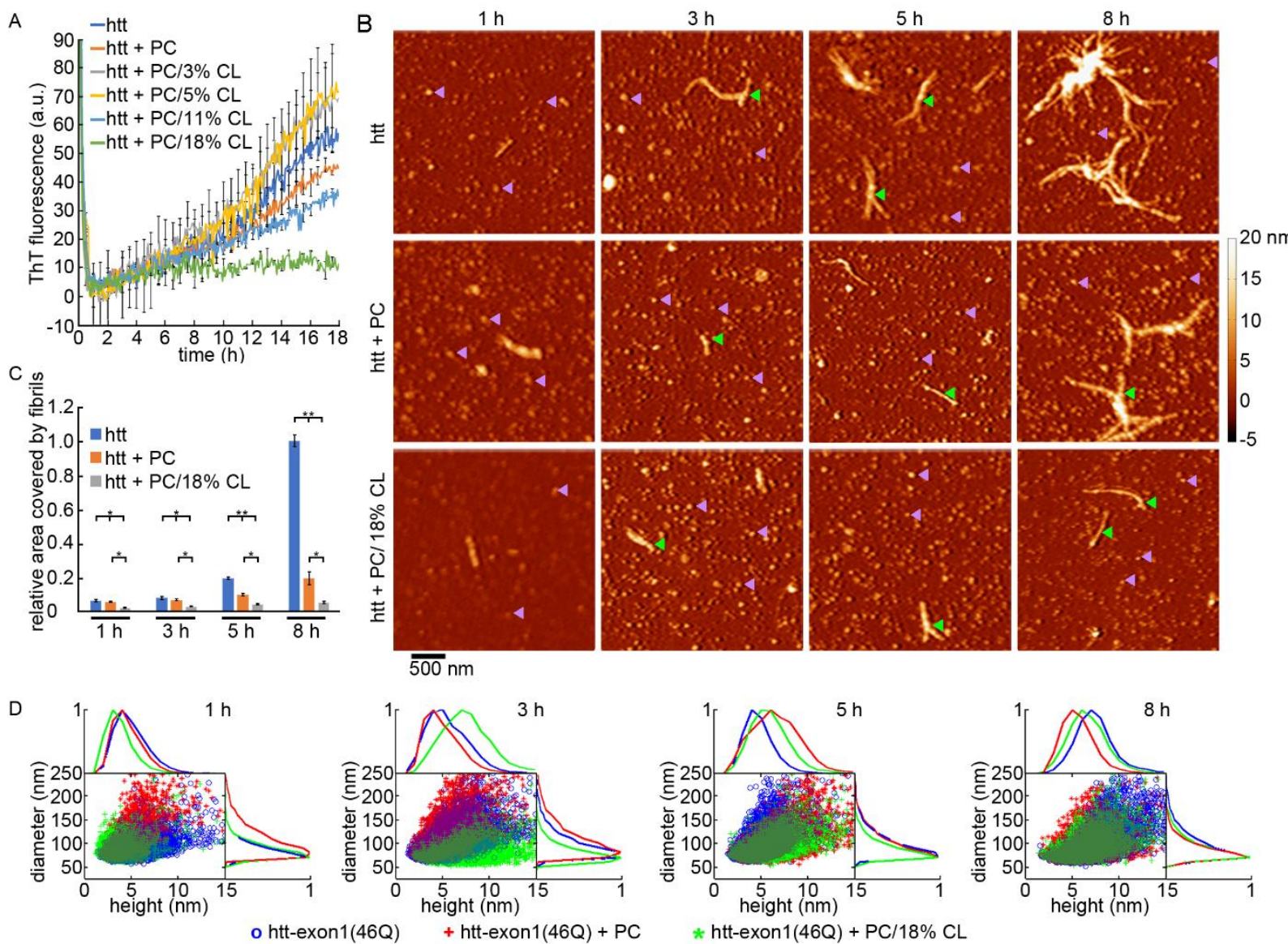

Figure 2.5. Cardiolipin impacts htt aggregation. (A) ThT analysis of the aggregation of htt-exon1(46Q) in the presence of PC vesicles with varying amounts of CL. Error bars represent SEM and are provided every $0.5 h$ (B) Representative AFM images of $h$ tt exon 1(46Q) incubated alone, with PC vesicles, or with PC vesicles with $18 \%$ CL. (C) Analysis of the surface area covered by fibrils as a function of time. Error bars represent SEM. * represents $p<0.05$ and ${ }^{* *}$ represents $p<0.01$. (D) Correlation plots of average diameter vs height of all oligomeric aggregates of htt-exon 1(46Q) (blue), htt exon1(46Q) + PC (red), and $h$ tt exon1 (46Q) + PC/18\% CL (green). For each correlation plot, the height (top) and diameter (right) histograms are provided.

monitored in the presence of POPC vesicles that contained various amounts of CL (3$18 \%$ by moles) at a peptide lipid ratio of 1:10 (Fig. 2.5A). Background controls (vesicles alone) were also performed (Fig. 2.1). With pure POPC vesicles, the extent of fibril formation was slightly reduced $(15-20 \%$ after $14 \mathrm{~h})$ compared with control (no lipid vesicles). Initial increases in CL content (up to $5 \%$ ) enhanced fibrillization by $\sim 15-20 \%$. 
Further increasing CL content resulted in inhibition of fibril formation, as $11 \%$ and $18 \%$ CL content reduced the ThT signal by $\sim 40 \%$, and $\sim 80 \%$ respectively.

Next, htt-exon1(46Q) was incubated $\left(20 \mu \mathrm{M}, 37^{\circ} \mathrm{C}\right)$ with POPC vesicles or POPC vesicles with $18 \%$ exogenous $\mathrm{CL}$ and sampled for AFM analysis (Fig. 2.5B-C) as was performed with IMM and OMM vesicles. Htt-exon1(46Q) was incubated in the absence of lipid as a control. A few small fibrils were observed after $1 \mathrm{~h}$ for all three incubations; however, the presence of POPC and POPC $+18 \% \mathrm{CL}$ vesicles significantly reduced fibril formation with time. Vesicles containing $C L$ reduce the fibril load on mica to a larger extent (Fig. 2.5B-C). Morphological features of oligomers from these incubations with POPC or POPC $+18 \% \mathrm{CL}$ were similar to control (Fig. 2.5D). However, oligomers formed in the presence of POPC $+18 \% \mathrm{CL}$ vesicles displayed larger variation in their morphology at different time points. At $1 \mathrm{~h}$, htt oligomers associated with POPC $+18 \% \mathrm{CL}$ had a smaller, tighter distribution of size compared to the other incubations. At $3 \mathrm{~h}$, oligomers associated with POPC $+18 \% \mathrm{CL}$ had a clear proportion of taller oligomers that appeared in the correlation plots in a similar fashion to oligomers formed in the presence of IMM mimic vesicles (Fig. 2.2D), which had comparable CL content. This is the only time point at which these oligomers were statistically different $(p<0.05)$ in size compared with control. After this $3 \mathrm{~h}$ time point, oligomers formed in the presence of POPC $+18 \% \mathrm{CL}$ were indistinguishable from control.

2.3.3 Htt aggregation varies on the surface of OMM and IMM membrane mimics. While OMM and IMM membranes impacted htt aggregation as demonstrated by ThT and ex situ AFM analysis, both techniques monitor bulk aggregation within an incubation. That is, they do not distinguish between htt aggregation occurring on a membrane surface or 
in solution. As it has been reported that htt aggregation occurs via a unique mechanism on POPC/POPS lipid surfaces, ${ }^{20}$ in situ AFM was employed to directly observe httexon1(46Q) on supported OMM and IMM mimicking bilayers (Fig. 2.6). OMM or IMM mimic vesicles were directly injected into an AFM fluid cell, and the formation of a supported lipid bilayer by vesicle fusion on mica was observed via continuous imaging. While OMM vesicles readily formed a complete bilayer, IMM did not, as several holes remained in IMM supported membranes after several hours of imaging. While we typically confirm complete bilayer formation before exposure to proteins in this type of in situ AFM studies, ${ }^{34,35,54,55}$ these incomplete supported IMM mimic bilayers were exposed to httexon1(46Q). However, reference images and membrane mapping were utilized to avoid analyzing areas of the membrane that contained holes prior to protein injection. With that caveat stated, supported bilayers were exposed to htt-exon1(46Q) (final concentration of $10 \mu \mathrm{M}$ in the fluid cell), and the bilayers were continuously imaged to directly observe aggregation and morphological changes occurring at the bilayer surface.

Upon exposure to htt, small oligomers quickly developed on the OMM surface (within 6 minutes, Fig. 2.6A). Around these oligomers, granular regions of increases surface roughness developed. While not extensive, some fibrils developed within these granular regions. These fibrils were often highly branched and grew in length with time. Some oligomers appeared to transition into fibrils after prolonged exposure to OMM. With that said, the vast majority of aggregates observed on OMM appeared globular. Over time, these structures started to swell (both in height and area) leading to increased roughness of the membrane. These rough patches did not develop over the entire OMM surface even after $87 \mathrm{~min}$ of exposure to htt-exon1(46Q). That is, regions of unperturbed 
OMM bilayer persisted. An unperturbed OMM bilayer had an RMS roughness of 0.673 $\mathrm{nm}$. The granular regions were significantly rougher, and this roughness steadily increased with time, reaching $7.045 \mathrm{~nm}$ within 120 minutes (Fig. 2.6B).
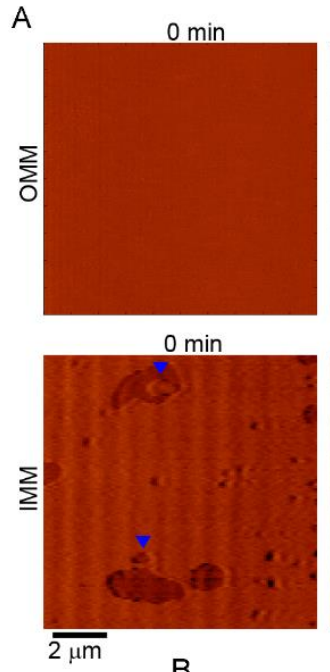

B

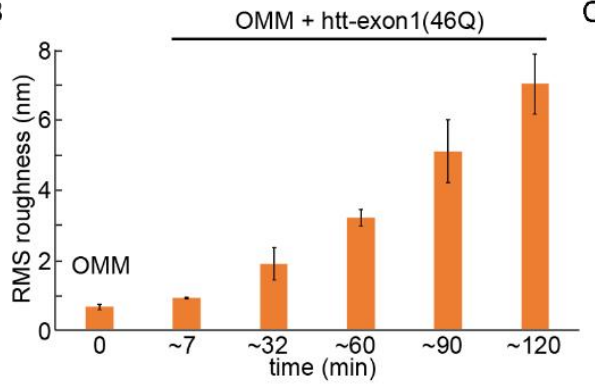

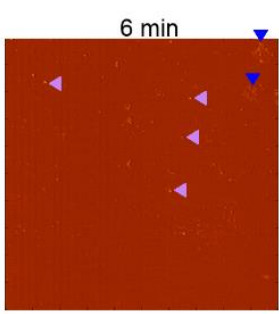

$6 \mathrm{~min}$
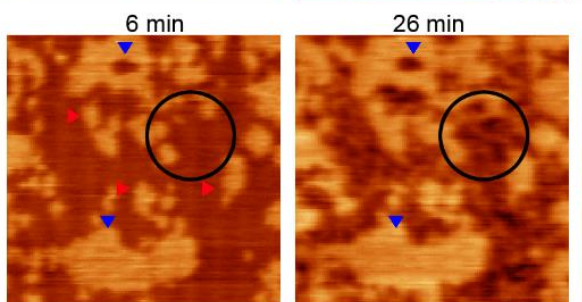

C

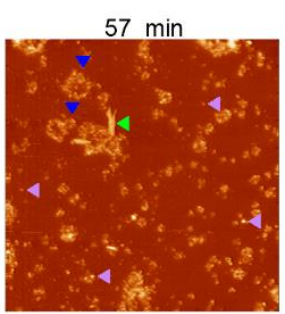

$46 \mathrm{~min}$
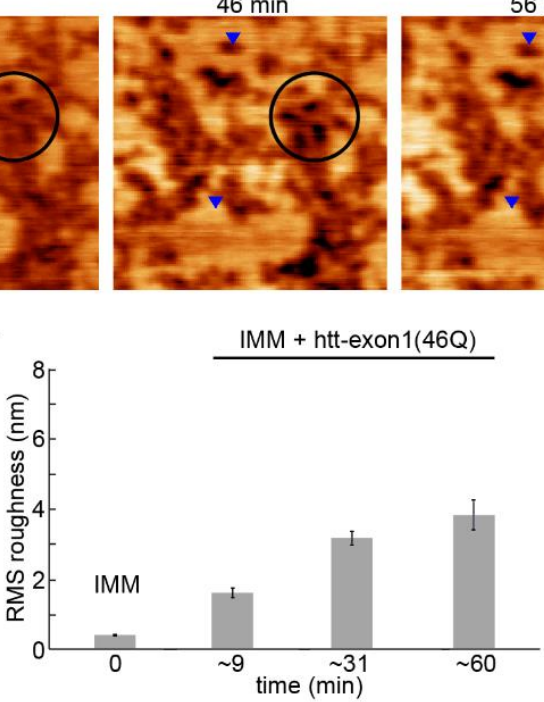

Figure 2.6. Huntingtin aggregation varies on the surfaces of OMM and IMM membrane mimics. (A) Time-resolved in situ AFM images of supported bilayers of OMM and IMM exposed to $10 \mu \mathrm{M}$ htt-exon1(46Q). Blue arrows indicated the same spot on sequential images. Green arrows indicate fibrils growing in sequential images. Purple arrows indicate oligomers. Red arrows indicate plateau-like regions developing on the IMM bilayer. Black circles highlight a region of the IMM bilayer that developed holes that spanned the entire membrane. Analysis of the RMS of disrupted regions of the surface of (B) OMM and (C) IMM bilayers before (0 $\mathrm{min}$ ) and after (7 to $120 \mathrm{~min}$ ) exposure to 10 $\mu M$ htt-exon1(46Q). Error bars represent SEM.

Morphological changes on the IMM bilayer upon exposure to htt-exon1(46Q) were markedly different (Fig. 2.6A and Fig. 2.7). Despite the difficulties of forming a complete 
bilayer prior to htt exposure, the holes in the IMM bilayer were quickly filled when htt was injected into the fluid cell, and these filled holes were associated with plateau-like domains that rose above the initial bilayer ( $\sim 3.838 \mathrm{~nm}$ in height) surface and spread to a broader area than the initial hole. While these plateau-like domains formed on regions of the IMM bilayer that contained an established hole prior to exposure to htt, these domains formed even in areas that did not initially contain any holes. These morphological changes on IMM bilayers developed more rapidly compared to those observed on OMM bilayers. That is, these plateau-like regions quickly enveloped the surface. Between the plateau-like domains, relatively smooth bilayer was observed for the first few minutes, however, these spaces quickly roughened with a granular morphology, often resulting in the development of large bilayer defects that extend all the way through the bilayer, exposing mica. Interestingly, discrete htt aggregates, oligomers, or fibrils, were typically not observed on the IMM membrane. A freshly prepared IMM mimic supported bilayer had an RMS roughness of $0.420 \mathrm{~nm}$, but upon exposure this significantly increased (Fig. 2.6B). The RMS roughness changes caused by exposure to htt-exon1(46Q) was typically larger on IMM compared to OMM at similar time points. However, due to the aggressive morphological changes observed on IMM, extending experiment on IMM beyond $\sim 60$ min was not feasible. Taken together, htt aggregation proceeds via distinct pathways on either OMM or IMM, resulting in distinct morphological changes of the bilayers. 

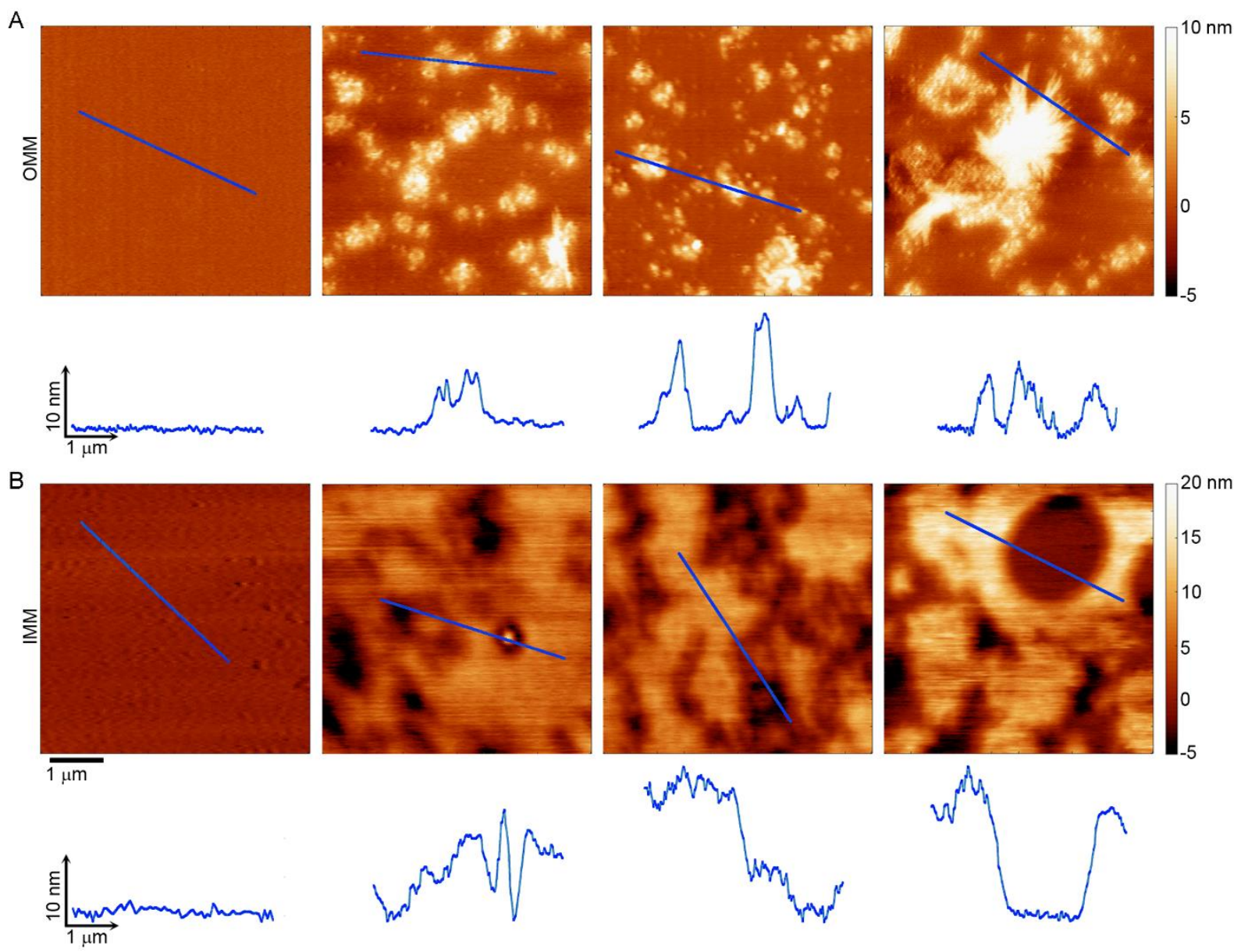

Figure 2.7. Additional in situ AFM images of supported (A) OMM and (B) IMM bilayers exposed to $10 \mu \mathrm{M}$ htt-exon1(46Q). The blue lines correspond to the height profiles directly below each image.

2.3.4 OMM, IMM, and CL containing vesicles do not destabilize preformed htt fibrils. It has been reported that some lipid systems can destabilize amyloid fibrils, causing them to refold into monomers or other aggregate species. ${ }^{56,57}$ More specifically, CL can refold $\alpha$-synuclein fibrils. ${ }^{58}$ With this in mind, the ability of OMM, IMM, POPC, and POPC $+18 \% \mathrm{CL}$ vesicles to destabilize htt fibrils was investigated (Fig. 2.8). Htt-exon1(46Q) was incubated at $30 \mu \mathrm{M}$ for $8 \mathrm{~h}$ to allow for the formation of fibrils. The presence of fibrils was confirmed by AFM. Then, these fibrils were diluted (final concentration of $20 \mu \mathrm{M}$ ) with neat buffer, OMM, IMM, POPC, or POPC $+18 \%$ CL vesicles with htt to lipid ratios of 1:10, 
1:20, and 1:30. The stability of these fibrils was tracked for $24 \mathrm{~h}$ with ThT fluorescence normalized to the control htt fibrils diluted in neat buffer (Fig. 2.8A). At all htt to lipid ratios, each lipid system was unable to destabilize fibrils as the relative ThT signals remained constant. To further validate that fibrils were stable in the presence of these lipid systems, the incubations were also sampled after 1 and $8 \mathrm{~h}$ for analysis by a filter trap assay and AFM (Fig. 2.8B-C). Serial dilutions (1.2, 0.6, $0.3 \mu \mathrm{g}$ htt) of each incubation were boiled in SDS before being drawn through a NCM membrane $(0.45 \mu \mathrm{m}$ pore size $)$ under vacuum, trapping large, SDS insoluble fibrils.
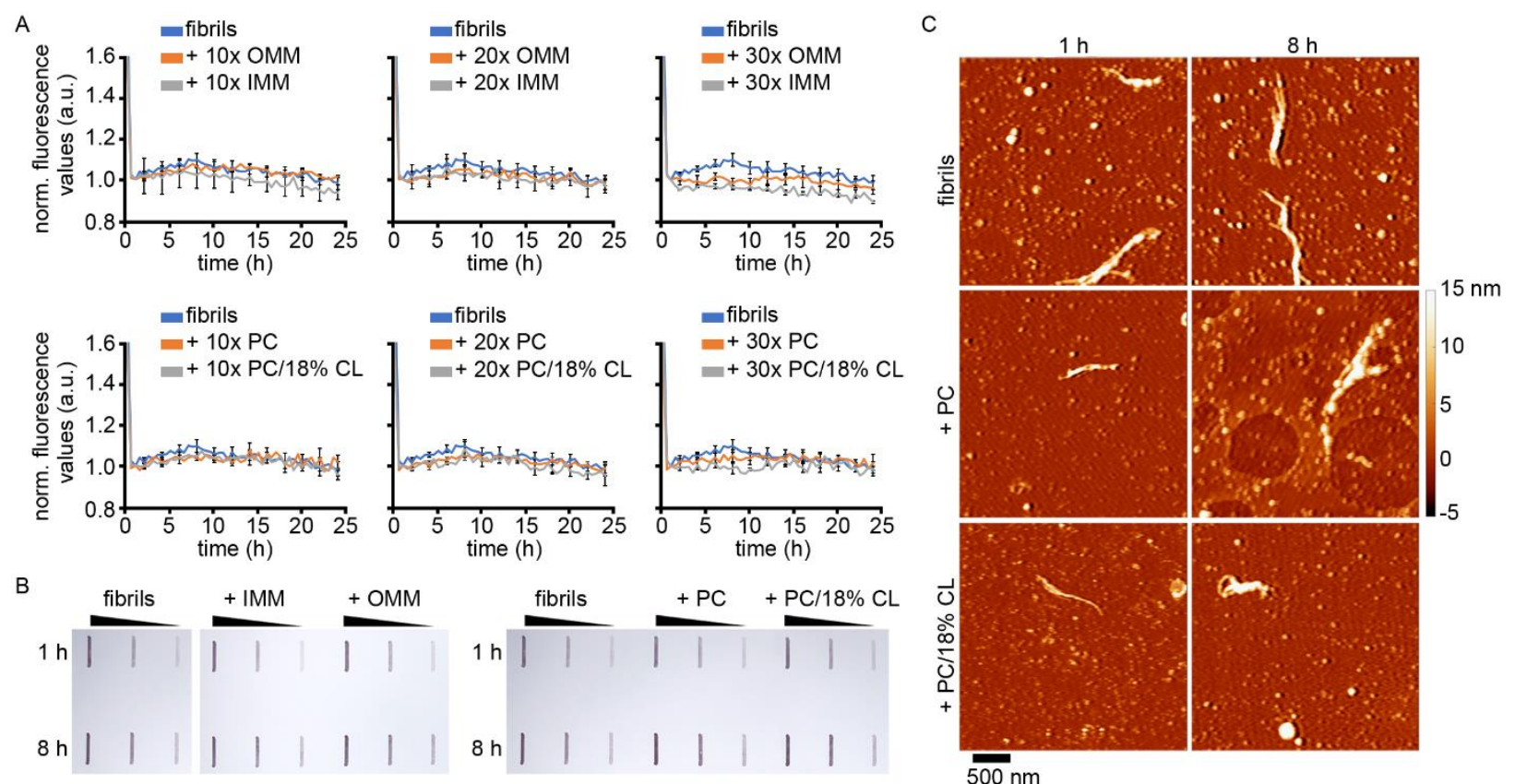

Figure 2.8. OMM, IMM, and CL do not disaggregate preformed htt fibrils. (A) Preformed fibrils were incubated alone and with lipid vesicles of OMM, IMM, POPC, or POPC + $18 \% \mathrm{CL}$, and fibril stability was monitored by ThT fluorescence over $24 \mathrm{~h}$. Peptide to lipid ratio ranged from 1:10 to 1:30. (B) Filter trap assay to determine preformed fibril stability after 1 and $8 \mathrm{~h}$ of incubation in buffer or after exposure to vesicles of IMM, OMM, POPC, and POPC $+18 \% \mathrm{CL}$. Each condition was serially diluted and probed with the anti-htt antibody MW8. (C) AFM images of preformed fibrils alone and in the presence of POPC and $P O P C+18 \% C L$ vesicles. 
The membrane was probed with anti-htt antibody MW8 that targets the C-terminal residues of htt-exon1 and readily detects fibrils. ${ }^{59,60}$ Fibrils were readily detected with similar intensities by the filter trap for all conditions and time points. Additionally, similar amounts of fibrils were detected in AFM images, confirming that IMM, IMM, POPC, and POPC vesicles containing CL do not readily destabilize htt fibrils for at least $24 \mathrm{~h}$.

\subsubsection{Mitochondrial-enriched fractions inhibit fibril formation. With OMM and IMM}

mimic vesicles and bilayers heavily influencing htt aggregation, the impact of tissuederived (from murine brain or bovine liver) mitochondrial-enriched fractions (MEFs) on htt aggregation was investigated. While the isolated mitochondria were often non-functional, these fractions consisted predominately of mitochondrial lipids and proteins. To determine how the presence of these mitochondria components impact htt aggregation, httexon1(46Q) was incubated $\left(20 \mu \mathrm{M}, 37^{\circ} \mathrm{C}\right)$ in the presence and absence of these MEFs. Control incubations of MEFs without htt were also carried out. The MEF concentration was approximated by measuring total protein content of each purification, and all experiments were performed at $1.4 \mathrm{mg} / \mathrm{ml}$ mitochondrial protein content. Aliquots were taken from these incubations and deposited on mica after 1, 3, 5, and $8 \mathrm{~h}$ of incubation for AFM analysis. Initially, htt-exon1(46Q) incubations contained a variety of small oligomers, but after $3 \mathrm{~h}$ of incubation short fibrils appeared (Fig. 2.9A). At 5 and $8 \mathrm{~h}$, fibrils were more abundant and longer. Fibrils were not observed from incubation of httexon1(46Q) mixed with MEFs derived from murine brain over $5 \mathrm{~h}$ of incubation. After $8 \mathrm{~h}$, only a few short fibrils appeared, but abundant oligomer-like features were observed at all time points (Fig. 2.9A). However, control incubations of murine brain derived MEFs alone also contained features reminiscent of htt oligomers (Fig. 2.9A). 
A
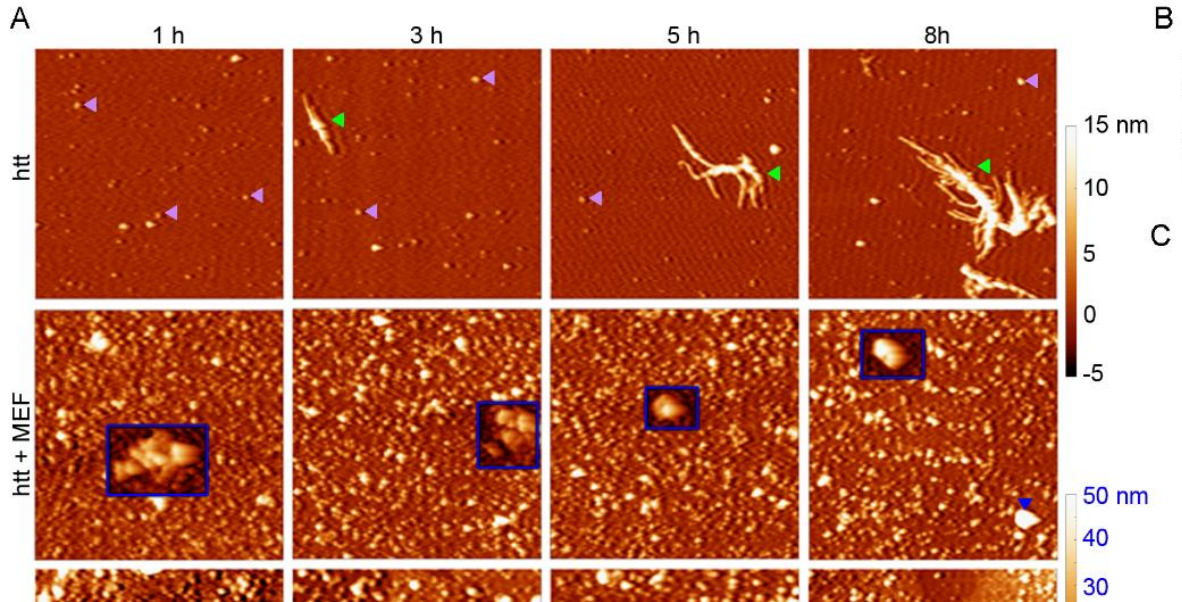

B
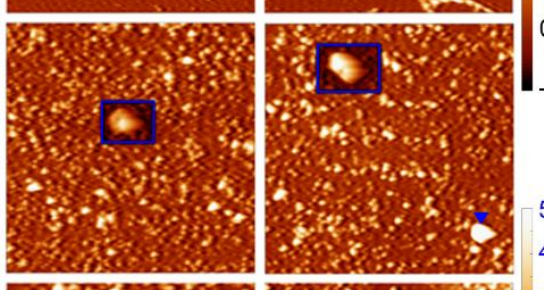

C

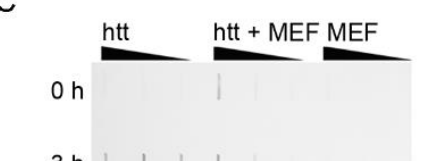

$3 \mathrm{~h}$

$5 \mathrm{~h}$

$$
8 \mathrm{~h} \mid
$$

D

घhtt $(1.2 \mu \mathrm{g}) \quad \mathbb{S} h t t(0.6 \mu \mathrm{g}) \quad$ खhtt $(0.3 \mu \mathrm{g})$ whtt $(1.2 \mu \mathrm{g})+\mathrm{MEF} \approx \mathrm{htt}(0.6 \mu \mathrm{g})+\mathrm{MEF}$ shtt $(0.3 \mu \mathrm{g})+\mathrm{MEF}$

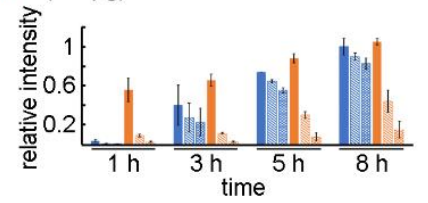

G

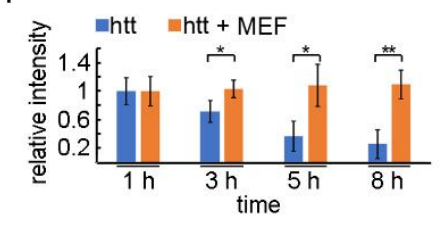

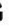

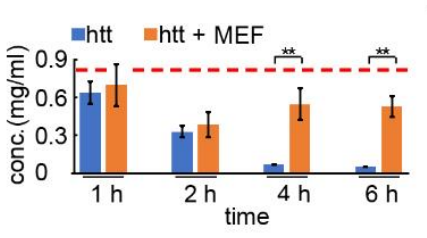

$\mathrm{H}$

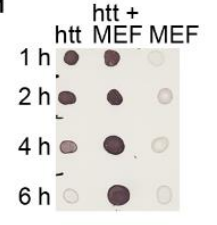

Figure 2.9. The impact of MEFs derived from murine brains on htt-exon1(46Q) aggregation. (A) Representative ex situ AFM images from incubations of htt-exon1(46Q) $(20 \mu M)$ with and without MEFs at various time points. Control images of MEFs alone are also presented. Due to large height differences between htt aggregates and mitochondria, the blue boxes in each image indicate areas using the colormap labeled with blue font. Examples of fibrils (green arrows), oligomers (purple arrows), and mitochondria (blue arrows) are marked. (B) Area of fibrils covered by htt-exon1(46Q) and htt-exon1(46Q) + MEFs in (A) reported relative to the maximum area covered by fibrils formed by httexon1(46Q) at $8 \mathrm{~h}$. (C) Filter trap assay investigating aggregation of htt-exon1(46Q) in the presence and absence of MEFs. Serial dilutions (1.2, 0.6 and $0.3 \mu \mathrm{g}$ of $\mathrm{htt}$ exon1(46Q)) for the htt alone and with MEFs, as well as the volume equivalents of the MEF alone control, were deposited on NCM (0.45 $\mu \mathrm{m}$ pore size) and probed with the antihtt antibody MW8. (D) Densitometry analysis of the slot blot assay in (C) taken across three independent experiments and normalized to the $1.2 \mu \mathrm{ght}$ alone sample at $8 \mathrm{~h}$. (E) Dot blot of the same experimental systems investigated in (C) were performed. $2 \mu L$ aliquots of each incubation were deposited on NCM $(0.1 \mu \mathrm{m}$ pore size) and probed with the anti-htt antibody MW1 to detect monomeric mutant htt. (F) Densitometry analysis of the dot blot assay in $(E)$ taken across three independent experiments and normalized to the $0 \mathrm{~h}$ time point for htt and htt $+M E F$ samples respectively. (G \& H) Determination of monomer remaining in the supernatent after centrifugation of $20 \mu \mathrm{M}$ htt-exon(46Q) with 
and without MEFs by $(G)$ a Bradford assay and $(H)$ dot blot analysis. The dashed red line in $(G)$ at $0.8 \mathrm{mg} / \mathrm{mL}$ indicates the initial concentration of GST-htt fusion protien in the incubation. The time points represent when the monomer concentration was measured. The monomer concentration of the htt + MEFs samples were corrrected for residual protein backgrounds associated with MEFs. The dot blots in $(H)$ were probed with MW1. All error bars represent standard error. ${ }^{*}$ represents $p<0.05$, and ${ }^{* *}$ represents $p<0.01$.

Morphological differences between these oligomer-like features observed in MEF alone controls and those observed in htt-exon1(46Q) incubated with MEFs will be analyzed later. In incubations of MEFs with and without htt, large mitochondria were observed by AFM at each time point. A similar pattern of aggregation was observed for incubation experiments performed with bovine liver derived MEFs (Fig. 2.10A). That is, httexon1(46Q) alone readily formed fibrils, but in the presence of MEFs, fibrils were not observed at any time point. To quantify this observation, all AFM images were analyzed, as previously described for studies with lipid vesicles, to determine the fibril load on mica for each condition. In this analysis, both mitochondria and fibrils met the larger than 9,000 $\mathrm{nm}^{2}$ criteria. As a result, the identified (by the Matlab script) structures were visually inspected to distinguish fibrils from mitochondrial features. The analysis of fibril load was normalized to the maximum fibril content associated with the htt-exon1(46Q) aggregation control at $8 \mathrm{~h}$, as it contained the largest fibril content (Fig. 2.9B, Fig. 2.10B). Whether incubated with brain or liver derived MEFs, the fibril load on mica was significantly reduced at all time points. It is possible that the presence of htt-exon1(46Q) could damage mitochondria presented in the MEF. As the mitochondria present in the MEFs were not consistently functional, the number and size of mitochondria observed in AFM images was evaluated to determine if this was impacted by exposure to htt. However, no significant changes were observed between mitochondria incubated in the presence or absence of htt-exon1(46Q) (Fig. 2.11). 
A
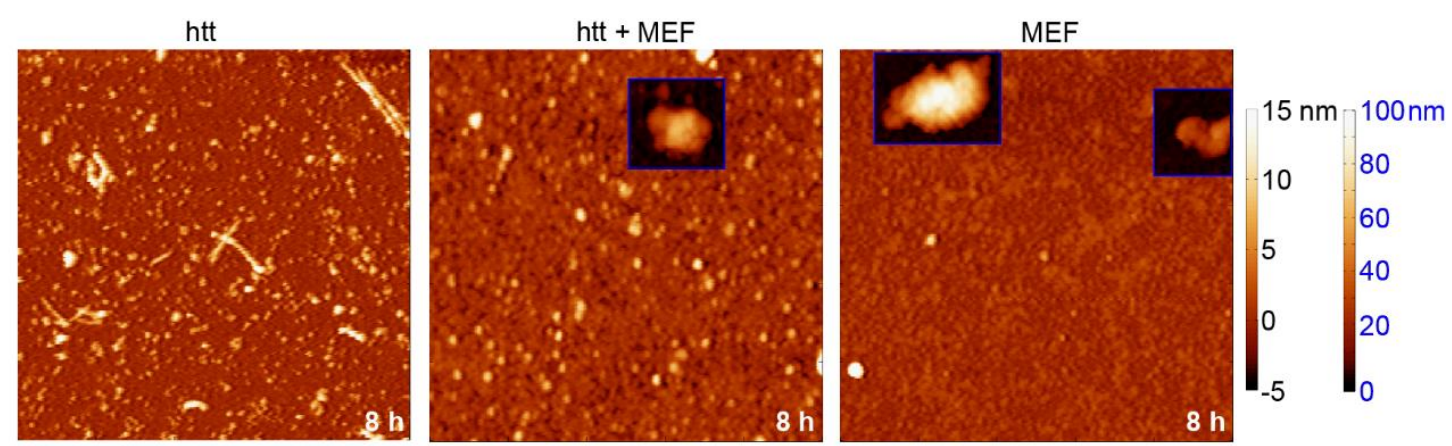

$\overline{500 \mathrm{~nm}}$

B

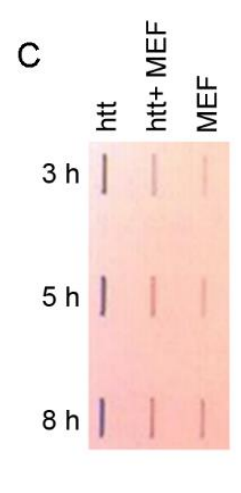

Figure 2.10. The impact of MEFs derived from bovine liver on htt-exon1(46Q) aggregation. (A) Representative ex situ AFM images of aliquots of htt-exon1(46Q) (20 $\mu M)$ with and without MEFs taken after $8 \mathrm{~h}$ of incubation. An image of control incubations of MEFs incubated alone is also presented. Due to the large height differences between $h t t$ aggregates and mitochondria, the blue boxes in each image indicate areas using the colormap labeled with blue font. (B) Analysis of the area covered by fibrils. These values are normalized to the fibril coverage in the $\mathrm{htt}$ alone sample at $8 \mathrm{~h}$. Black arrows indicate that no fibrils were identified. (C) Filter trap assay investigating aggregation of httexon1(46Q) in the presence and absence of MEFs at various time points and probed with the anti-htt antibody MW8. 


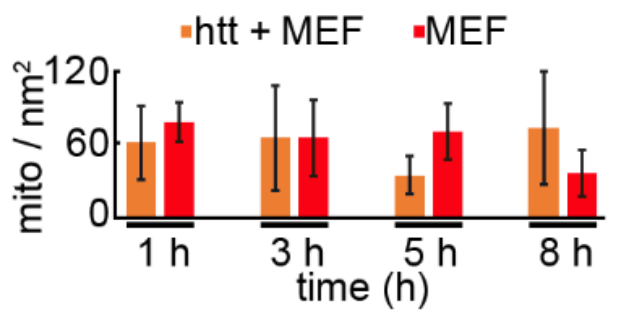

Figure 2.11. Analysis of identifiable mitochondria populations incubated with (orange) and without (red) htt-exon1(46Q). Mitochondria in AFM images taken at various time points were identified with automated scripts written in Matlab. These scripts generated AFM images with identifed mitochondria labeled, allowing for visual verification. Features reatained for this analysis were taller than $7.5 \mathrm{~nm}$ and occupited a surface area of $0.08 \mu m^{2}$.

While AFM experiments suggested that the presence of MEFs inhibited fibril formation, there was the possibility that the presence of MEFs may interfere with fibrils adhering to the mica surface. As a result, a filter trap assay was performed to validate the AFM results. To probe for fibril formation, aliquots were taken from the different incubations, boiled in SDS, and serially diluted, $(1.2,0.6,0.3 \mu \mathrm{g}$ htt or the volume equivalent for the MEF alone control) and probed via a filter trap assay using the anti-htt antibody MW8. For htt-exon1(46Q) incubated alone, no fibrils were trapped at $0 \mathrm{~h}$; however consistent with the AFM assay, significant htt was detected after 3,5 , and $8 \mathrm{~h}$ of incubation, and this was slightly reduced with serial dilution of the aliquot (Fig. 2.9C-D). When htt-exon1(46Q) was incubated with murine brain-derived MEFs, a significant amount of htt was detected by the filter trap at each time point for the non-diluted aliquot, but unlike htt alone, htt content significantly decreased upon serial dilution. With control mitochondria, no htt was detected as expected. At first glance, this assay suggests htt formed fibrils in the presence of MEFs; however, an alternative interpretation seems plausible. Mitochondria are large enough to not pass through the filter trap. Exogenous htt bound to mitochondria would be concentrated on the organelle surface and detectable 
by this assay. In comparison to free floating htt fibrils, serial dilution would impact the amount of htt detected in the presence of mitochondria due to concentrating effect of the organelle surface. As a result, htt observed with mitochondria is not necessarily fibrillar but is bound to the organelle. However, boiling with SDS should breakdown intact mitochondria in the MEF, allowing them to pass. To determine if mitochondria were being retained by the filter trap assay, membranes were stained using a primary antibody for VDAC, a mitochondrial protein (Fig. 2.12). If mitochondria are completely broken down by boiling in SDS, VDAC would pass through the filter. However, VDAC would be detected if mitochondria are retained in the filter trap. Based on this test, mitochondria were retained by the filter trap, and this retention was reduced via serial dilution.

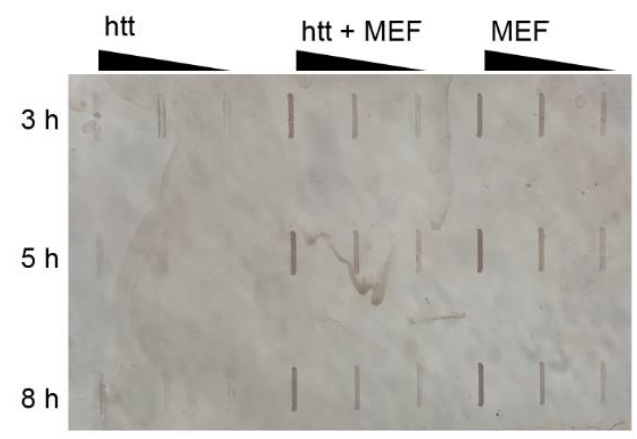

Figure 2.12. Immunochemical analysis of murine brain derived MEFs with and without htt-exon1(46Q) at various time points. Serial dilutions (2.0, 1.0 and $0.5 \mu \mathrm{g})$ of $1.4 \mathrm{mg} / \mathrm{ml}$ MEF (BSA equivalents) which are of comparable volumes to 1.2, 0.6 and $0.3 \mu \mathrm{g}$ of $20 \mu \mathrm{M}$ htt exon1(46Q)) were boiled in SDS and deposited on NCM ((0.45 $\mu \mathrm{m}$ pore size) at various time points and probed with VDAC polyclonal antibody.

To further clarify if fibrils formed in the presence of MEFs, a dot blot assay was performed. Small aliquots of the different incubation conditions were deposited on NCM $(0.1 \mu \mathrm{m}$ pore size $)$ and probed with the anti-htt antibody MW1, which targets polyQ but can't detect the compact amyloid core of fibrils. ${ }^{59}$ This allows for detecting non-fibrillar 
forms of htt. For incubations of htt-exon1(46Q) alone, significant, non-fibrillar htt was detected at $1 \mathrm{~h}$ (Fig. 2.9E-F); however, detectable htt dramatically decreased with time as fibrils formed. When htt-exon1(46Q) was incubated with murine brain derived MEFs, similar htt staining was observed at each time point, confirming suppression of fibril formation. Similar patterns were observed in both filter trap and dot blot assays performed with bovine liver-derived MEFs (Fig. 2.13).

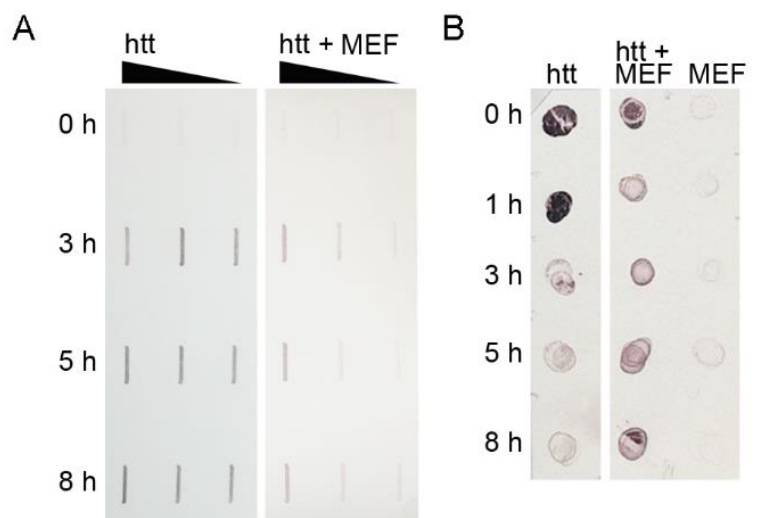

Figure 2.13. Immunochemical analysis of htt-exon1(46Q) without and with bovine liverderived MEFs at various time points. (A) Filter trap assay investigating htt aggregation of htt-exon1(46Q) in the presence and absence of MEFs. Serial dilutions (1.2, 0.6 and 0.3 $\mu \mathrm{g}$ ht exon1(46Q)) and the volume equivalents of htt exon1(46Q) + MEFs and MEFs alone were deposited on NCM (0.45 $\mu \mathrm{m}$ pore size) at various time points and probed with the anti-htt antibody MW8. (B) To detect non-fibrillar aggregates, complementary dot blots of the same experimental systems investigated in (A) were performed (B). $2 \mu L$ aliquots of each incubation were deposited on NCM $(0.1 \mu \mathrm{m}$ pore size) and probed with the antihtt antibody MW1 to detect monomeric htt.

As the filter trap assay suggested htt was binding to mitochondria, a variation of a standard monomer loss assay was performed to assess the extent to which htt localized to the mitochondria present in the MEF (Fig. 2.9G). Htt-exon1(46Q) was incubated (20 $\mu \mathrm{M}$ or $0.84 \mathrm{mg} / \mathrm{mL}$ of GST-htt fusion protein, $37^{\circ} \mathrm{C}$ ) in the presence and absence of freshly isolated murine-derived MEFs. MEFs were also incubated without htt-exon1(46Q) as a control. After $0,1,3$, and $5 \mathrm{~h}$ of incubations, aliquots were removed and centrifuged at 
$20,000 \times g$ for 40 minutes to pellet aggregated htt and mitochondria present in the MEFs. The supernatant was collected, and protein concentration determined by a Bradford assay. As a result of this procedure, the supernatant concentration was measured $\sim 1 \mathrm{~h}$ after sampling the incubations, and these adjusted times are reported on Fig. 2.9G (1, 2, 4 , and $6 \mathrm{~h})$. In addition, the protein concentration of the htt-exon1(46Q) + MEFs incubations were corrected for residual protein associated with mitochondria alone controls. At $1 \mathrm{~h}$, a significant and similar amount of monomer loss was observed for httexon1(46Q) incubated with and without MEFs compared to the initial htt concentration. However, by 4 and $6 \mathrm{~h}$, the monomer loss associated with htt-exon1(46Q) incubated in the absence of MEFs continued to decrease, consistent with extensive aggregation. When incubated with mitochondria, the monomer concentration stabilized at later time points, suggesting that the monomer loss was due to htt pelleting with mitochondria and that aggregation was suppressed. While corrected with control experiments, residual mitochondria protein content was still a complication. As a result, a second method to measure htt-exon1(46Q) concentration in the supernatant was employed for validation. The collected supernatants were evaluated with a dot blot assay using MW1 to specifically measure $h t t$ content (Fig. $2.9 \mathrm{H}$ ). The detectable htt-exon1(46Q) in the supernatant decreased with time in a manner consistent with fibrillization. With MEFs, the htt-exon1(46Q) detected in the supernatant was stable across all time points.

The Bradford assay measured free protein content $(\sim 0.55 \pm 0.06 \mathrm{mg} / \mathrm{mL}$ across all time points) in the supernatants of the control mitochondria alone incubation even though mitochondria pellet under the centrifugal conditions were used. To determine if residual contents in the supernatant of these MEF samples underlie the observed reduction in htt 
fibrillization, ThT assays were performed with $20 \mu \mathrm{M}$ htt-exon1(46Q) in the presence and absence of supernatant from centrifuged $(20,000 \times \mathrm{g}$ for 40 minutes) MEFs derived from both murine brain and bovine liver (Fig. 2.14). The volume of supernatant added to these ThT assays was equivalent to the volume that would have been required to achieve the $1.4 \mathrm{mg} / \mathrm{ml}$ mitochondrial protein content prior to centrifugation. Neither of the supernatants significantly altered the ThT signal, confirming that the entity reducing fibrillization was present in the pellet.

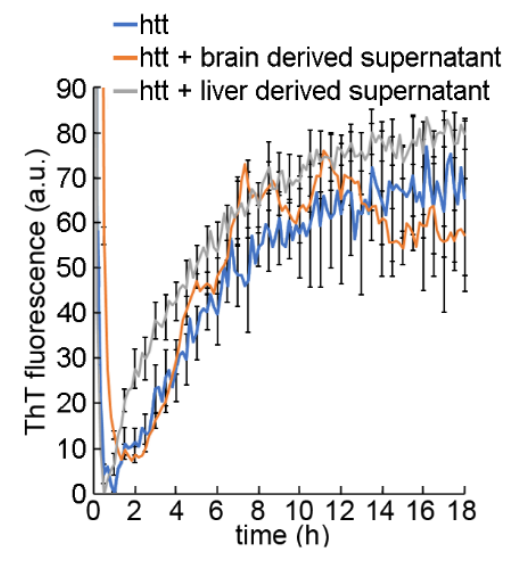

Figure 2.14. Control ThT assay demonstrating the impact of residual material in the supernatant of centrifuged MEFs on htt-exon1(46Q) (20 $\mu$ M) fibril formation.

Supernatant from both murine brain-derived and bovine liver-derived MEFs were tested and obtained after centrifgation for $40 \mathrm{~min}$ at 20,000 $\times$ g. Error bars represent SEM.

2.3.6 Mitochondria influence htt oligomer formation. Having observed fibril suppression by MEFs, we returned to the AFM images to determine if there were any morphological differences associated with oligomers formed in the presence of these fractions. Oligomer morphology was analyzed using the same criteria employed to study incubations with lipid vesicles described above. Correlation plots of diameter and height were constructed, as well as histograms for both dimensions (Fig. 2.15). Complicating this analysis is that a great number of features in the MEFs alone met these criteria. While 
there was some overlap in the correlation plots for these MEF-related features with oligomers of htt-exon1(46Q) observed in control (no MEFs), the background features associated with mitochondria (in the absence of htt) from both murine brain and bovine liver had smaller observed heights (mode typically in the range of 2-3.5 nm, Fig. 2.15 and Fig. 2.16). In general, many htt oligomers were morphologically distinct from MEF backgrounds as visualized in the correlation plots. With this stated, oligomers of httexon1(46Q) in the absence of MEFs were distinguishable from those formed in the presence of MEFs due to differences predominately in height. That is oligomers formed by htt alone at early time points had mode heights of $\sim 4-5.5 \mathrm{~nm}$ that could shift to larger sizes by $8 \mathrm{~h}(\sim 6-7 \mathrm{~nm})$, but oligomers formed in the presence of MEFs had mode heights of 7-11 $\mathrm{nm}$ after 1,3 , and $5 \mathrm{~h}$ of incubation. These oligomers of htt in the presence of MEFs became smaller at $8 \mathrm{~h}$ (mode height of $\sim 5-7 \mathrm{~nm}$ ). In that regard, htt oligomers formed in the presence of MEFs were significantly larger than htt oligomers formed in the absence of MEFs ( $p<0.05$, students T-test) for all time points except after $8 \mathrm{~h}$ of incubation. These differences in oligomer morphology were more pronounced with bovine liver-derived fractions (Fig. 2.15), with oligomers formed in the presence of these MEFs having significantly larger height distributions $(p<0.05)$ compared to htt controls after 3 and $5 \mathrm{~h}$ of incubation. Importantly, the correlation plots make it clear that these features are not the same as those observed in the MEF alone controls (background), and features similar to those observed in the control are not observed when mitochondria are incubated with htt. For example, the height distributions of measured features of samples with htt-exon1(46Q) (in the presence and absence of brain- or liver-derived MEFs) were typically significantly $(p<0.05)$ larger than the background features observed in images 
of mitochondria alone samples. The only exception is the $8 \mathrm{~h}$ time point with brain derived mitochondria. This suggests that the background debris associated with MEFs is accumulating with htt, resulting in distinct globular aggregates. Due to this difference in heights, oligomers formed in the presence of MEFs appear as a distinct population across the bottom of the correlation plots, similar to those observed in some time points with IMM and $\mathrm{PC}+18 \% \mathrm{CL}$ vesicles described earlier
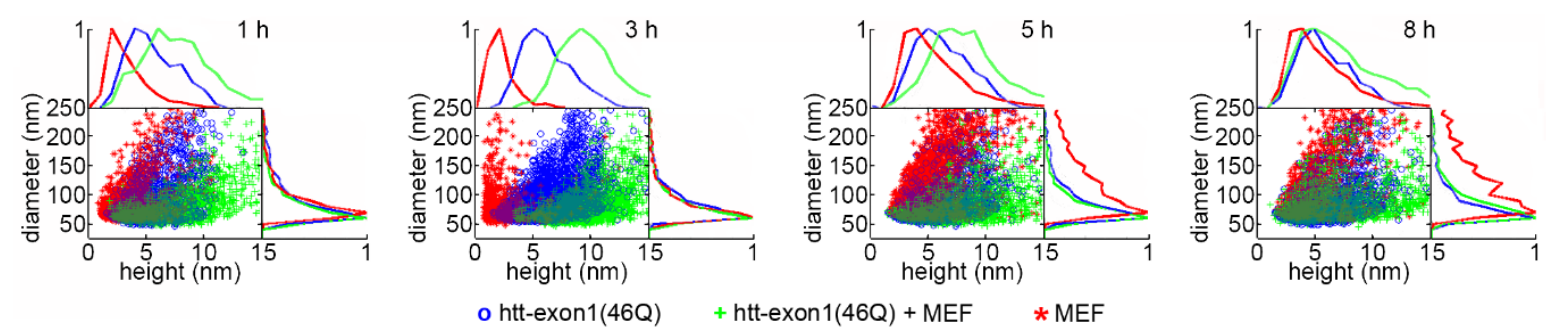

Figure 2.15. Murine-derived brain MEFs alter oligomer morphology. Correlation plots of average diameter vs height of all oligomer-sized aggregates of htt exon1(46Q) (blue), htt exon1(46Q) + MEFs (green), and MEFs alone (red). For each correlation plot, the height (top) and diameter (right) histograms are also provided.

2.3.7 Mitochondria-enriched fractions do not destabilize preformed htt fibrils. As with the pure lipid systems, the ability of MEFs to destabilize htt fibrils was determined. As MEFs interfered with ThT assays, this ability was only assessed by filter trap assays using MW8 and AFM (Fig. 2.17). Preformed htt-exon1(46Q) fibrils were diluted (final concentration of $20 \mu \mathrm{M}$ ) with neat buffer or MEFs (mitochondrial protein content equivalent of $1.4 \mathrm{mg} / \mathrm{ml})$. 

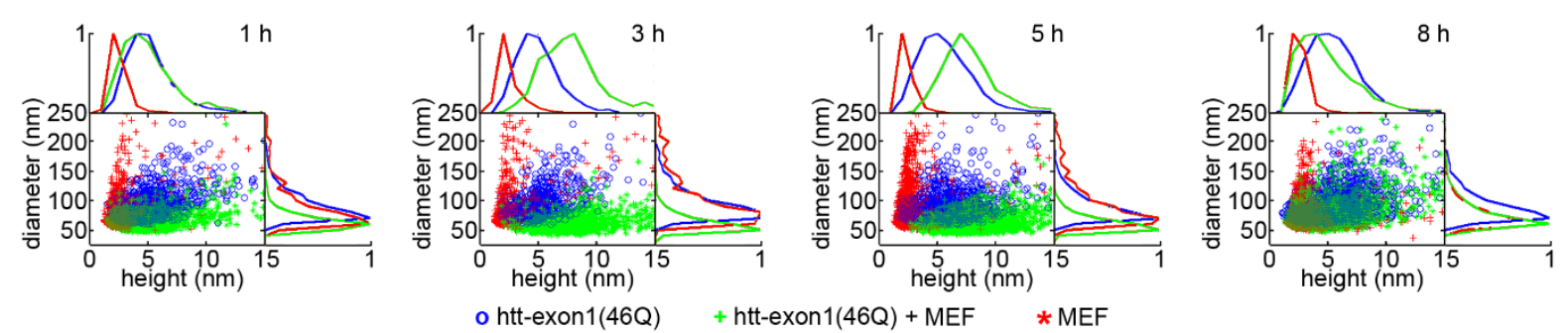

Figure 2.16. Analysis of oligomers formed by htt-exon1(46Q) in the presence and absence of MEFs derived from bovine liver. Correlation plots of average diameter vs height of all oligomer-sized aggregates of htt-exon1(46Q) (blue), htt-exon1(46Q) + MEFs (green), and MEFs (red). For each correlation plot, the height (top) and diameter (right) histograms are also provided.

Serial dilutions $(1.2,0.6,0.3 \mu \mathrm{g} \mathrm{htt})$ of each condition were prepared for the filter trap assay after 1 and $8 \mathrm{~h}$ of incubation. Fibrils were readily detected with similar intensities by the filter trap for the fibril control and fibrils + MEFs incubations at both time points. A control MEF alone did not elicit any response in the filter trap assay. Additionally, AFM images confirmed the stability of preformed htt fibrils exposed to MEFs. Similar to mitochondria incubated with fresh, unaggregated htt, there was no apparent alteration in mitochondria morphology upon exposure to preformed htt fibrils. 


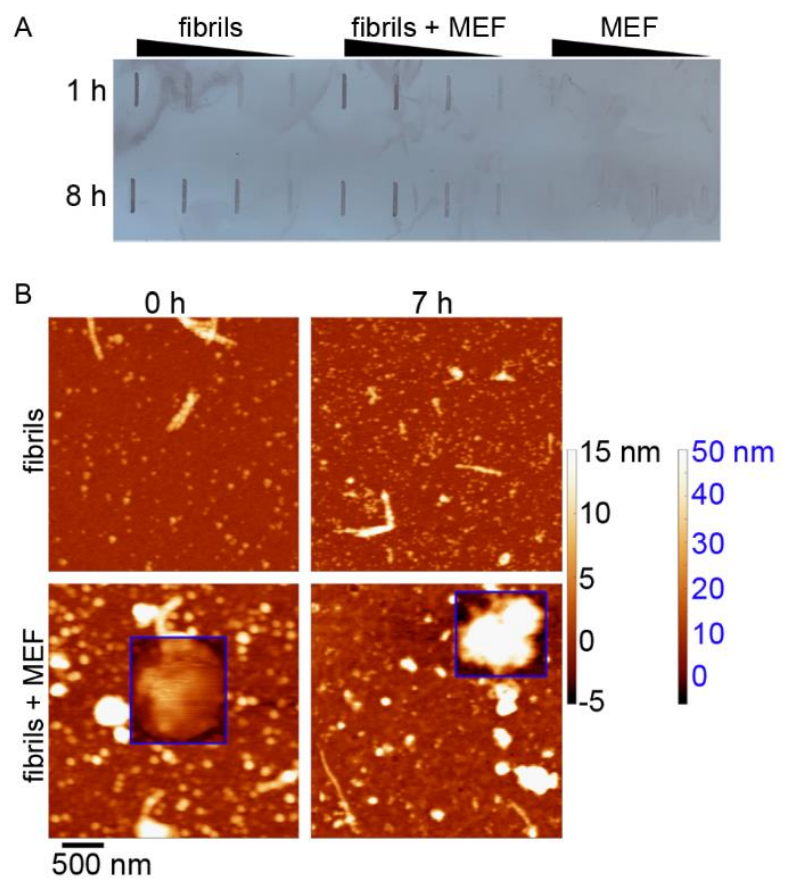

Figure 2.17. MEFs do not destabilize preformed htt fibrils. (A) Preformed fibrils were incubated alone and with MEFs, and fibril stability was monitored by a filter trap assay after 1 and $8 \mathrm{~h}$. Each condition was serially diluted and probed with the anti-htt antibody MW8. (B) AFM images of preformed fibrils alone and in the presence of MEFs. Due to large height differences between htt aggregates and mitochondria, the blue boxes in each image indicate areas using the colormap labeled with blue font.

\subsection{Discussion}

Amyloid formation is implicated as a common factor across many neurodegenerative diseases, including HD, Alzheimer's disease (AD), and Parkinson's disease (PD). The aggregation processes associated with amyloids are dependent on a variety of cellular factors, including the presence of lipid membranes. ${ }^{16}$ Beyond influencing aggregation, membranes represent potential targets for amyloid induced damage and disruption. In HD, htt-induced membrane damage may underlie observed dysfunction of membranous organelles. ${ }^{27,28,36,43}$ Here, the direct impact of model mitochondrial membranes and MEFs on htt aggregation was determined. To glean the 
role of mitochondrial membranes on htt aggregation, htt aggregation was monitored in the presence of OMM or IMM mimics, and membrane mimics impeded fibril formation with IMM having the larger impact. In addition, IMM mimics promoted a unique htt oligomer at early time points. The influence of the mitochondrial membrane mimics appeared to be $\mathrm{CL}$ content dependent, and removing $\mathrm{CL}$ from the IMM mimic compromised its ability to impede fibril formation. Therefore, the role of $\mathrm{CL}$ in htt aggregation was systematically determined, and two regimes were observed. Simple membranes with low CL content slightly promoted htt fibrillization; whereas, higher, more physiologically relevant $\mathrm{CL}$ content inhibited fibrilization. Htt aggregation was also directly observed on the surface of OMM and IMM mimic bilayers. Exposure to htt altered membrane morphology differently between these two lipid systems. Discrete oligomers and some fibrils formed on OMM that were associated with granular regions of membrane disruption. Discrete aggregates did not readily appear on IMM, but the membrane developed distinct regions displaying a plateau-like morphology. In between these plateaus, the membrane roughened, and holes spanning the entire bilayer appeared. The presence of MEFs inhibited htt fibril formation in a similar fashion to the mimic systems and also promoted the formation of a distinct htt oligomer. However, neither the purified lipid systems nor the MEFs destabilized preformed htt fibrils. Collectively, these results highlight the complexity of htt-exon1 aggregation in the presence of mitochondria and that the two distinct membranes of mitochondria impact htt aggregation in unique ways with CL playing a prominent role.

Full length htt is a large, 350 kDa protein; however, this study utilized a htt-exon1 fragment with an expanded polyQ domain as a model system. Use of exon1 as a model 
system is physiologically justified and is extensively used. Expression of mutant htt-exon1 in rodent and primate models captures aggregation patterns and elicits neurological defects similar to HD. ${ }^{61-63}$ Indeed, a variety of $\mathrm{N}$-terminal htt fragments are produced in HD brains. ${ }^{61-64}$ These cleavage products are produced by caspase, calpain and other protease activity, which precedes pathogenesis. ${ }^{65,66} \mathrm{~N}$-terminal fragments appear to be more potently cytotoxic and aggregation prone, compared to full-length htt. ${ }^{67-69}$ Importantly, N-terminal fragments (including exon1) of mutant htt associate with mitochondria, resulting in dysfunction.

While mitochondria inhibited htt fibrillization and promoted unique oligomeric species, simplified model systems implicated $C L$ as playing a major role in these phenomena. The impact of CL-containing membranes on htt aggregation was dependent on $\mathrm{CL}$ content. With low CL content $(<5 \%)$, htt fibrilization was slightly accelerated, but when CL content was high ( $>5 \%$ ), fibril formation was inhibited in a dose dependent manner. Recently, molecular dynamic simulations of ternary lipid systems demonstrated a similar CL-content dependent impact on membrane fluidity ${ }^{70}$. That is, membrane fluidity was reduced at low CL content $(2-10 \%)$; whereas, $15 \% \mathrm{CL}$ content greatly increased fluidity. ${ }^{70}$ This suggests that the varied impact of CL on physicochemical properties of membranes, such as fluidity, influence the varied interaction of htt with membranes observed here. While the influence of lipid membranes on htt aggregation has been well appreciated, ${ }^{71}$ the $\mathrm{htt} /$ lipid interaction is a complicated phenomenon that is highly dependent on lipid content. The impact of a variety of model lipid systems on htt aggregation has been explored, ranging from pure lipid systems ${ }^{20,72,73}$ to brain lipid extracts. For example, POPC/POPS lipid vesicles accelerate fibril formation, ${ }^{20}$ but total 
brain lipid extract (TBLE) inhibits fibrillization. ${ }^{72}$ Altering the composition of TBLE with cholesterol, sphingomyelin, or GM1 further modifies htt aggregation. ${ }^{34,35}$ In particular, exogenous cholesterol promoted the formation of plateau-like domains ${ }^{34}$ similar to those observed on IMM mimic bilayers.

$\mathrm{CL}$ is a phospholipid that is unique in both shape and location, that is, it is almost exclusively found in the mitochondria with higher abundance in the IMM. CL is comprised of one anionic head and four lipid tails. The disparity between the sizes of the head and tails of CL causes it to adopt a conical shape inducing bilayer curvature. The distinctive properties of this lipid contribute to its role in maintaining IMM and OMM integrity and as a regulator of mitochondria dynamics, ${ }^{74-76}$ either through formation of microdomains ${ }^{77}$ or recruitment of other proteins. ${ }^{51}$ The permeabilization of mitochondrial membranes by amyloid-forming proteins, e.g. $\alpha$-synuclein and the $\beta$-amyloid peptide, is associated with other neurodegenerative diseases and appears mediated by CL content..$^{50}$ In particular, $\mathrm{CL}$ promotes ion pore formation by $\alpha$-synuclein ${ }^{78}$ and recruits $\alpha$-synuclein to mitochondrial surfaces causing fragmentation. ${ }^{79} \mathrm{Htt}$ obtained from mouse brain lysates have a particularly high affinity for $\mathrm{CL},{ }^{80}$ which may in part be due to htt's ability to preferentially bind curved membranes ${ }^{81}$ which are promoted by CL. ${ }^{82,83}$ In addition, the lipid binding domain, $\mathrm{Nt17}$, has a net positive charge that may further stabilize binding with negatively charged CL headgroups, as the ability of $\mathrm{Nt17}$ to bind lipids is sensitive to electrostatic interactions. ${ }^{73}$

With htt aggregating to different extents in the presence of and producing unique morphological changes in IMM and OMM model systems, the extent of exposure of these membranes in intact mitochondria to htt is an important consideration. The interaction of 
htt with the OMM is well established, and htt remains attached to the OMM even during mitochondrial isolation. ${ }^{24,43,84-86}$ The ability of htt to reach the intermembrane space and reach the IMM remains unresolved. By probing immunoblots with anti-htt antibodies of mitochondrial subfractions, htt was found to be associated with both the IMM and OMM; however, a complete separation between these two membranes may not be possible with this method. ${ }^{43}$ In the same study, analysis of susceptibility to trypsin digest suggested that htt was not internalized into mitochondria and is associated with the cytosolic face of the OMM in a conformation that is partially resistant to trypsin proteolysis. Additional reports using varying combinations of limited trypsin digest, selective OMM permeabilization, and alkali treatment provide conflicting evidence with regard to the ability of htt to enter the intermembrane space. In support of htt reaching the IMM, mutant htt appears to specifically binds to subunits of the mitochondrial inner membrane translocase subunit TIM23, ${ }^{39,44}$ which would require htt crossing the OMM. With regard to these TIM23 studies, trypsin proteolysis assays coupled with selective permeabilization of the OMM suggested htt localization into the intermembrane space of mitochondria. ${ }^{44}$ However, recent reports using similar assays did not detect htt in the intermembrane space. ${ }^{87}$ Due to our inability to verify mitochondrial functionality in our MEFs, it is likely that the integrity of the OMM was compromised. As a result, our study does not provide additional data with regard to this controversy. Rather, we demonstrate that the presence of mitochondrial surfaces alters htt aggregation and that this is predominately impacted by $C L$ content. While the $C L$ content is appreciably smaller in the OMM than the IMM, CL content can be enriched in the OMM during mitophagy. ${ }^{88,89}$ 
The lipid composition of the IMM and OMM facilitate many functions of mitochondria..$^{90}$ These phospholipids provide structural integrity, and mitochondrial proteins are located and function within these membranes. ${ }^{90}$ Wild type and mutant htt fragments localize to mitochondria, but only mutant htt negatively impacts mitochondria trafficking ${ }^{24}$ and protein import. ${ }^{39}$ In HD, the ability of mitochondria to maintain calcium homeostasis is significantly impaired, ${ }^{25}$ which could be caused by htt directly damaging mitochondrial membranes as observed here on OMM and IMM mimics. Beyond lipids, there are several htt/mitochondrial protein interactions that play a role in $\mathrm{HD}$. Htt binds proteins that target mitochondria to promote mitophagy ${ }^{40}$ or fragmentation. ${ }^{45} \mathrm{~A}$ prominent mitochondrial fission protein, dynamin related protein (DRP1) is overexpressed in HD models, altering mitochondrial dynamics in HD. ${ }^{45-47} \mathrm{CL}$, which is implicated here in modifying htt aggregation, also stimulates DRP1 activity. ${ }^{91,92}$ Mutant htt also localizes to the intermembrane space of mitochondria, binding an IMM protein (TIM23) and eliciting proteome imbalance. ${ }^{44}$ 


\section{REFERENCES}

(1) MacDonald, M. E., Ambrose, C. M., Duyao, M. P., Myers, R. H., Lin, C., Srinidhi, L., Barnes, G., Taylor, S. A., James, M., Groot, N., MacFarlane, H., Jenkins, B., Anderson, M. A., Wexler, N. S., Gusella, J. F., Bates, G. P., Baxendale, S., Hummerich, H., Kirby, S., North, M., Youngman, S., Mott, R., Zehetner, G., Sedlacek, Z., Poustka, A., Frischauf, A. M., Lehrach, H., Buckler, A. J., Church, D., Doucette-Stamm, L., O'Donovan, M. C., Riba-Ramirez, L., Shah, M., Stanton, V. P., Strobel, S. A., Draths, K. M., Wales, J. L., Dervan, P., Housman, D. E., Altherr, M., Shiang, R., Thompson, L., Fielder, T., Wasmuth, J. J., Tagle, D., Valdes, J., Elmer, L., Allard, M., Castilla, L., Swaroop, M., Blanchard, K., Collins, F. S., Snell, R., Holloway, T., Gillespie, K., Datson, N., Shaw, D., and Harper, P. S. (1993) A novel gene containing a trinucleotide repeat that is expanded and unstable on Huntington's disease chromosomes. Cell 72, 971-983.

(2) Myers, R. H., Vonsattel, J. P., Stevens, T. J., Cupples, L. A., Richardson, E. P., Martin, J. B., and Bird, E. D. (1988) Clinical and neuropathologic assessment of severity in huntington's disease. Neurology 38, 341-347.

(3) Li, S. H., and Li, X. J. (1998) Aggregation of N-terminal huntingtin is dependent on the length of its glutamine repeats. Human Molecular Genetics 7, 777-782.

(4) Martindale, D., Hackam, A., Wieczorek, A., Ellerby, L., Wellington, C., Mccutcheon, K., Singaraja, R., Kazemi-esfarjani, P., Devons, R., Kim, S. U., Bredesen, D. E., Tufaro, F., and Hayden, M. R. (1998) Length of huntingtin and its polyglutamine tract influences localization and frequency of intracellular aggregates. Nature Genetics 18, 150-154.

(5) Yamamoto, A., Lucas, J. J., and Hen, R. (2000) Reversal of neuropathology and motor dysfunction in a conditional model of Huntington's disease. Cell 101, 57-66.

(6) Ona, V. O., Li, M., Vonsattel, J. P. G., John Andrews, L., Khan, S. Q., Chung, W. M., Frey, A. S., Menon, A. S., Li, X. J., Stieg, P. E., Yuan, J., Penney, J. B., Young, A. B., Cha, J. H. J., and Friedlander, R. M. (1999) Inhibition of caspase-1 slows disease progression in a mouse model of Huntington's disease. Nature 399, 263-267.

(7) Arrasate, M., Mitra, S., Schweitzer, E. S., Segal, M. R., and Finkbeiner, S. (2004) Inclusion body formation reduces levels of mutant huntingtin and the risk of neuronal death. Nature 431, 805-910.

(8) Kim, Y. E., Hosp, F., Mann, M., Hayer-hartl, M., and Hartl, F. U. (2016) Soluble Oligomers of PolyQ-Expanded Huntingtin Target a Multiplicity of Key Cellular Factors Article Soluble Oligomers of PolyQ-Expanded Huntingtin Target a Multiplicity of Key Cellular Factors. Molecular Cell 63, 951-964.

(9) Olshina, M. A., Angley, L. M., Ramdzan, Y. M., Tang, J., Bailey, M. F., Hill, A. F., and Hatters, D. M. (2010) Tracking mutant huntingtin aggregation kinetics in cells reveals three major populations that include an invariant oligomer pool. Journal of Biological Chemistry 285, 21807-21816. 
(10) Sahl, S. J., Weiss, L. E., Duim, W. C., Frydman, J., and Moerner, W. E. (2012) Cellular inclusion bodies of mutant huntingtin exon 1 obscure small fibrillar aggregate species. Scientific Reports 2, 1-7.

(11) Nagai, Y., Inui, T., Popiel, H. A., Fujikake, N., Hasegawa, K., Urade, Y., Goto, Y., Naiki, H., and Toda, T. (2007) A toxic monomeric conformer of the polyglutamine protein. Nature Structural and Molecular Biology 14, 332-340.

(12) Lajoie, P., and Snapp, E. L. (2010) Formation and toxicity of soluble polyglutamine oligomers in living cells. PLOS ONE 5, e15245.

(13) Drombosky, K. W., Rode, S., Kodali, R., Jacob, T. C., Palladino, M. J., and Wetzel, R. (2018) Mutational analysis implicates the amyloid fibril as the toxic entity in Huntington's disease. Neurobiology of Disease 120, 126-138.

(14) Bhattacharyya, A., Thakur, A. K., Chellgren, V. M., Thiagarajan, G., Williams, A. D., Chellgren, B. W., Creamer, T. P., and Wetzel, R. (2006) Oligoproline effects on polyglutamine conformation and aggregation. Journal of Molecular Biology 355, 524-535.

(15) Thakur, A. K., Jayaraman, M., Mishra, R., Thakur, M., Chellgren, V. M., Byeon, I.-J. L., Anjum, D. H., Kodali, R., Creamer, T. P., Conway, J. F., Gronenborn, A. M., and Wetzel, R. (2009) Polyglutamine disruption of the huntingtin exon $1 \mathrm{~N}$ terminus triggers a complex aggregation mechanism. Nature structural \& molecular biology 16, 380-9.

(16) Burke, K. A., Yates, E. A., and Legleiter, J. (2013) Biophysical insights into how surfaces, including lipid membranes, modulate protein aggregation related to neurodegeneration. Frontiers in Neurology 4, 1-17.

(17) Jayaraman, M., Kodali, R., Sahoo, B., Thakur, A. K., Mayasundari, A., Mishra, R., Peterson, C. B., and Wetzel, R. (2012) Slow amyloid nucleation via a-helix-rich oligomeric intermediates in short polyglutamine-containing huntingtin fragments. Journal of Molecular Biology 415, 881-899.

(18) Mishra, R., Jayaraman, M., Roland, B. P., Landrum, E., Fullam, T., Kodali, R., Thakur, A. K., Arduini, I., and Wetzel, R. (2012) Inhibiting the nucleation of amyloid structure in a huntingtin fragment by targeting a-helix-rich oligomeric intermediates. Journal of Molecular Biology 415, 900-917.

(19) Michalek, M., Salnikov, E. S., and Bechinger, B. (2013) Structure and Topology of the Huntingtin $1-17$ Membrane Anchor by a Combined Solution and Solid-State NMR Approach. Biophysj 105, 699-710.

(20) Pandey, N. K., Isas, J. M., Rawat, A., Lee, R. v., Langen, J., Pandey, P., and Langen, R. (2017) The 17-residue-long $N$ terminus in huntingtin controls step-wise aggregation in solution and on membranes via different mechanisms. Journal of Biological Chemistry 293, 2597-2605. 
(21) Atwal, R. S., Xia, J., Pinchev, D., Taylor, J., Epand, R. M., and Truant, R. (2007) Huntingtin has a membrane association signal that can modulate huntingtin aggregation, nuclear entry and toxicity. Human Molecular Genetics 16, 2600-2615.

(22) Peters, M. F., Nucifora, F. C., Kushi, J., Seaman, H. C., Cooper, J. K., Herring, W. J., Dawson, V. L., Dawson, T. M., and Ross, C. A. (1999) Nuclear targeting of mutant huntingtin increases toxicity. Molecular and Cellular Neurosciences 14, 121-128.

(23) Gutekunst, C., Li, S., Yi, H., Mulroy, J. S., Kuemmerle, S., Jones, R., Rye, D., Ferrante, R. J., Hersch, S. M., and Li, X. (1999) Nuclear and Neuropil Aggregates in Huntington 's Disease : Relationship to Neuropathology 19, 2522-2534.

(24) Orr, A. L., Li, S., Wang, C.-E., Li, H., Wang, J., Rong, J., Xu, X., Mastroberardino, P. G., Greenamyre, J. T., and Li, X.-J. (2008) N-Terminal Mutant Huntingtin Associates with Mitochondria and Impairs Mitochondrial Trafficking. Journal of Neuroscience 28, 27832792.

(25) Panov, A. v., Gutekunst, C.-A., Leavitt, B. R., Hayden, M. R., Burke, J. R., Strittmatter, W. J., and Greenamyre, J. T. (2002) Early mitochondrial calcium defects in Huntington's disease are a direct effect of polyglutamines. Nature Neuroscience 5, 731736.

(26) Shirendeb, U., Reddy, A. P., Manczak, M., Calkins, M. J., Mao, P., Tagle, D. A., and Reddy, P. H. (2011) Abnormal mitochondrial dynamics, mitochondrial loss and mutant huntingtin oligomers in Huntington's disease: Implications for selective neuronal damage. Human Molecular Genetics 20, 1438-1455.

(27) Ueda, M., Li, S., Itoh, M., Wang, M., Hayakawa, M., Islam, S., Nakagawa, K., Chen, H., and Nakagawa, T. (2016) Biochemical and Biophysical Research Communications Expanded polyglutamine embedded in the endoplasmic reticulum causes membrane distortion and coincides with Bax insertion. Biochemical and Biophysical Research Communications 474, 259-263.

(28) Liu, K. Y., Shyu, Y. C., Barbaro, B. A., Lin, Y. T., Chern, Y., Thompson, L. M., Shen, C. K. J., and Marsh, J. L. (2015) Disruption of the nuclear membrane by perinuclear inclusions of mutant huntingtin causes cell-cycle re-entry and striatal cell death in mouse and cell models of Huntington's disease. Human Molecular Genetics 24, 1602-1616.

(29) Qin, Z. H., Wang, Y., Sapp, E., Cuiffo, B., Wanker, E., Hayden, M. R., Kegel, K. B., Aronin, N., and DiFiglia, M. (2004) Huntingtin Bodies Sequester Vesicle-Associated Proteins by a Polyproline-Dependent Interaction. Journal of Neuroscience 24, 269-281.

(30) Kegel, K. B., Kim, M., Sapp, E., McIntyre, C., Castano, J. G., Aronin, N., and DiFiglia, M. (2000) Huntingtin expression stimulates endosomal-lysosomal activity, endosome tubulation, and autophagy. Journal of Neuroscience 20, 7268-7278.

(31) Kegel, K. B., Sapp, E., Yoder, J., Cuiffo, B., Sobin, L., Kim, Y. J., Qin, Z. H., Hayden, M. R., Aronin, N., Scott, D. L., Isenberg, G., Goldmann, W. H., and DiFiglia, M. (2005) 
Huntingtin associates with acidic phospholipids at the plasma membrane. Journal of Biological Chemistry 280, 36464-36473.

(32) Burke, K. A., Kauffman, K. J., Umbaugh, C. S., Frey, S. L., and Legleiter, J. (2013) The interaction of polyglutamine peptides with lipid membranes is regulated by flanking sequences associated with huntingtin. Journal of Biological Chemistry 288, 1499315005.

(33) Burke, K. A., Yates, E. A., and Legleiter, J. (2013) Amyloid-forming proteins alter the local mechanical properties of lipid membranes. Biochemistry 52, 808-817.

(34) Gao, X., Campbell, W. A., Chaibva, M., Jain, P., Leslie, A. E., Frey, S. L., and Legleiter, J. (2016) Cholesterol Modifies Huntingtin Binding to, Disruption of, and Aggregation on Lipid Membranes. Biochemistry 55, 92-102.

(35) Chaibva, M., Gao, X., Jain, P., Campbell, W. A., Frey, S. L., and Legleiter, J. (2017) Sphingomyelin and GM1 Influence Huntingtin Binding to, Disruption of, and Aggregation on Lipid Membranes. ACS Omega 3, 273-285.

(36) Saha, I., Mishra, A., Hartl, F. U., and Baumeister, W. (2017) In Situ Architecture and Cellular Interactions of PolyQ Inclusions Article In Situ Architecture and Cellular Interactions of PolyQ Inclusions. Cell 171, 179-187.

(37) Choo, Y. S., Johnson, G. V. W., MacDonald, M., Detloff, P. J., and Lesort, M. (2004) Mutant huntingtin directly increases susceptibility of mitochondria to the calcium-induced permeability transition and cytochrome c release. Human Molecular Genetics 13, 14071420.

(38) Rockabrand, E., Slepko, N., Pantalone, A., Nukala, V. N., Kazantsev, A., Marsh, J. L., Sullivan, P. G., Steffan, J. S., Sensi, S. L., and Thompson, L. M. (2007) The first 17 amino acids of Huntingtin modulate its sub-cellular localization, aggregation and effects on calcium homeostasis. Human Molecular Genetics 16, 61-77.

(39) Yano, H., Baranov, S. V., Baranova, O. V., Kim, J., Pan, Y., Yablonska, S., Carlisle, D. L., Ferrante, R. J., Kim, A. H., and Friedlander, R. M. (2014) Inhibition of mitochondrial protein import by mutant huntingtin. Nature Neuroscience 17, 822-831.

(40) Guo, X., Sun, X., Hu, D., Wang, Y. J., Fujioka, H., Vyas, R., Chakrapani, S., Joshi, A. U., Luo, Y., Mochly-Rosen, D., and Qi, X. (2016) VCP recruitment to mitochondria causes mitophagy impairment and neurodegeneration in models of Huntington's disease. Nature Communications 7, 1-17.

(41) Jenkins, B. G., Koroshetz, W. J., Beal, M. F., and Rosen, B. R. (1993) Evidence for impairment of energy metabolism in vivo in Huntington's disease using localized 1H NMR spectroscopy. Neurology 43, 2689-2695. 
(42) Gu, M., Gash, M. T., Mann, V. M., Javoy-Agid, F., Cooper, J. M., and Schapira, A. H. V. (1996) Mitochondrial defect in Huntington's disease caudate nucleus. Annals of Neurology 39, 385-389.

(43) Choo, Y. S., Johnson, G. V. W., MacDonald, M., Detloff, P. J., and Lesort, M. (2004) Mutant huntingtin directly increases susceptibility of mitochondria to the calcium-induced permeability transition and cytochrome c release. Human Molecular Genetics 13, 14071420.

(44) Yablonska, S., Ganesan, V., Ferrando, L. M., Kim, J. H., Pyzel, A., Baranova, O. V., Khattar, N. K., Larkin, T. M., Baranov, S. V., Chen, N., Strohlein, C. E., Stevens, D. A., Wang, X., Chang, Y. F., Schurdak, M. E., Carlisle, D. L., Minden, J. S., and Friedlander, R. M. (2019) Mutant huntingtin disrupts mitochondrial proteostasis by interacting with TIM23. Proceedings of the National Academy of Sciences of the United States of America 116, 16593-16602.

(45) Song, W., Chen, J., Petrilli, A., Liot, G., Klinglmayr, E., Zhou, Y., Poquiz, P., Tjong, J., Pouladi, M. A., Hayden, M. R., Masliah, E., Ellisman, M., Rouiller, I., Schwarzenbacher, R., Bossy, B., Perkins, G., and Bossy-Wetzel, E. (2011) Mutant huntingtin binds the mitochondrial fission GTPase dynamin-related protein-1 and increases its enzymatic activity. Nature medicine 17, 377-82.

(46) Guo, X., Disatnik, M., Marie, S., Mochly-rosen, D., and Qi, X. (2013) Inhibition of mitochondrial fragmentation diminishes Huntington's disease - associated neurodegeneration Find the latest version: Inhibition of mitochondrial fragmentation diminishes Huntington' $s$ disease - associated neurodegeneration. Journal of Clinical Investigation 123, 5371-5388.

(47) Cherubini, M., Lopez-Molina, L., and Gines, S. (2020) Mitochondrial fission in Huntington's disease mouse striatum disrupts ER-mitochondria contacts leading to disturbances in $\mathrm{Ca} 2+$ efflux and Reactive Oxygen Species (ROS) homeostasis. Neurobiology of Disease 136, 104741.

(48) Kim, J., Moody, J. P., Edgerly, C. K., Bordiuk, O. L., Cormier, K., Smith, K., Flint Beal, M., and Ferrante, R. J. (2010) Mitochondrial loss, dysfunction and altered dynamics in Huntington's disease. Human Molecular Genetics 19, 3919-3935.

(49) Wacker, J. L., Zareie, M. H., Fong, H., Sarikaya, M., and Muchowski, P. J. (2004) $\mathrm{Hsp} 70$ and Hsp40 attenuate formation of spherical and annular polyglutamine oligomers by partitioning monomer. Nature Structural and Molecular Biology 11, 1215-1222.

(50) Camilleri, A., Zarb, C., Caruana, M., Ostermeier, U., Ghio, S., Högen, T., Schmidt, F., Giese, A., and Vassallo, N. (2013) Mitochondrial membrane permeabilisation by amyloid aggregates and protection by polyphenols. Biochimica et Biophysica Acta Biomembranes 1828, 2532-2543. 
(51) Lutter, M., Fang, M., Luo, X., Nishijima, M., Xie, X. S., and Wang, X. (2000) Cardiolipin provides specificity for targeting of tBid to mitochondria. Nature Cell Biology 2, 754-756.

(52) Burke, K. A., Godbey, J., and Legleiter, J. (2011) Assessing mutant huntingtin fragment and polyglutamine aggregation by atomic force microscopy. Methods 53, 275284.

(53) Levy, G. R., Shen, K., Gavrilov, Y., Smith, P. E. S., Levy, Y., Chan, R., Frydman, J., and Frydman, L. (2019) Huntingtin's N-Terminus Rearrangements in the Presence of Membranes: A Joint Spectroscopic and Computational Perspective. ACS Chemical Neuroscience 10, 472-481.

(54) Sedighi, F., Adegbuyiro, A., and Legleiter, J. (2020) SUMOylation Prevents Huntingtin Fibrillization and Localization onto Lipid Membranes. ACS Chemical Neuroscience 11, 328-343.

(55) Chaibva, M., Jawahery, S., Pilkington, A. W., Arndt, J. R., Sarver, O., Valentine, S., Matysiak, S., and Legleiter, J. (2016) Acetylation within the First 17 Residues of Huntingtin Exon 1 Alters Aggregation and Lipid Binding. Biophysical Journal 111, 349362.

(56) Martins, I. C., Kuperstein, I., Wilkinson, H., Maes, E., Vanbrabant, M., Jonckheere, W., Van Gelder, P., Hartmann, D., D'Hooge, R., De Strooper, B., Schymkowitz, J., and Rousseau, F. (2008) Lipids revert inert $A \beta$ amyloid fibrils to neurotoxic protofibrils that affect learning in mice. EMBO Journal 27, 224-233.

(57) Chaparro Sosa, A. F., De Oliveira Da Silva, S. M., Morgan, G. P., Schwartz, D. K., and Kaar, J. L. (2020) Mixed Phospholipid Vesicles Catalytically Inhibit and Reverse Amyloid Fibril Formation. Journal of Physical Chemistry Letters 11, 7417-7422.

(58) Ryan, T., Bamm, V. V., Stykel, M. G., Coackley, C. L., Humphries, K. M., JamiesonWilliams, R., Ambasudhan, R., Mosser, D. D., Lipton, S. A., Harauz, G., and Ryan, S. D. (2018) Cardiolipin exposure on the outer mitochondrial membrane modulates $\alpha$ synuclein. Nature Communications 9, 1-17.

(59) Lin, H. K., Boatz, J. C., Krabbendam, I. E., Kodali, R., Hou, Z., Wetzel, R., Dolga, A. M., Poirier, M. A., and Van Der Wel, P. C. A. (2017) Fibril polymorphism affects immobilized non-amyloid flanking domains of huntingtin exon1 rather than its polyglutamine core. Nature Communications 8, 1-12.

(60) Legleiter, J., Lotz, G. P., Miller, J., Ko, J., Ng, C., Williams, G. L., Finkbeiner, S., Patterson, P. H., and Muchowski, P. J. (2009) Monoclonal antibodies recognize distinct conformational epitopes formed by polyglutamine in a mutant huntingtin fragment. Journal of Biological Chemistry 284, 21647-21658.

(61) Davies, S. W., Turmaine, M., Cozens, B. A., DiFiglia, M., Sharp, A. H., Ross, C. A., Scherzinger, E., Wanker, E. E., Mangiarini, L., and Bates, G. P. (1997) Formation of 
neuronal intranuclear inclusions underlies the neurological dysfunction in mice transgenic for the HD mutation. Cell 90, 537-548.

(62) von Hörsten, S., Schmitt, I., Nguyen, H. P., Holzmann, C., Schmidt, T., Walther, T., Bader, M., Pabst, R., Kobbe, P., Krotova, J., Stiller, D., Kask, A., Vaarmann, A., RathkeHartlieb, S., Schulz, J. B., Grasshoff, U., Bauer, I., Vieira-Saecker, A. M. M., Paul, M., Jones, L., Lindenberg, K. S., Landwehrmeyer, B., Bauer, A., Li, X. J., and Riess, O. (2003) Transgenic rat model of Huntington's disease. Human Molecular Genetics 12, 617-624.

(63) Yang, S. H., Cheng, P. H., Banta, H., Piotrowska-Nitsche, K., Yang, J. J., Cheng, E. C. H., Snyder, B., Larkin, K., Liu, J., Orkin, J., Fang, Z. H., Smith, Y., Bachevalier, J., Zola, S. M., Li, S. H., Li, X. J., and Chan, A. W. S. (2008) Towards a transgenic model of Huntington's disease in a non-human primate. Nature 453, 921-924.

(64) Hoffher, G., Island, M. L., and Djian, P. (2005) Purification of neuronal inclusions of patients with Huntington's disease reveals a broad range of $\mathrm{N}$-terminal fragments of expanded huntingtin and insoluble polymers. Journal of Neurochemistry 95, 125-136.

(65) Wellington, C. L., Ellerby, L. M., Gutekunst, C. A., Rogers, D., Warby, S., Graham, R. K., Loubser, O., Van Raamsdonk, J., Singaraja, R., Yang, Y. Z., Gafni, J., Bredesen, D., Hersch, S. M., Leavitt, B. R., Roy, S., Nicholson, D. W., and Hayden, M. R. (2002) Caspase cleavage of mutant huntingtin precedes neurodegeneration in Huntington's disease. Journal of Neuroscience 22, 7862-7872.

(66) Miller, J. P., Holcomb, J., Al-Ramahi, I., de Haro, M., Gafni, J., Zhang, N., Kim, E., Sanhueza, M., Torcassi, C., Kwak, S., Botas, J., Hughes, R. E., and Ellerby, L. M. (2010) Matrix metalloproteinases are modifiers of huntingtin proteolysis and toxicity in Huntington's disease. Neuron 67, 199-212.

(67) Bates, G. (2003) Huntingtin aggregation and toxicity in Huntington's disease. Lancet $361,1642-1644$.

(68) Li, H., Li, S. H., Johnston, H., Shelbourne, P. F., and Li, X. J. (2000) Amino-terminal fragments of mutant huntingtin show selective accumulation in striatal neurons and synaptic toxicity. Nature Genetics 25, 385-389.

(69) DiFiglia, M., Sapp, E., Chase, K. O., Davies, S. W., Bates, G. P., Vonsattel, J. P., and Aronin, N. (1997) Aggregation of huntingtin in neuronal intranuclear inclusions and dystrophic neurites in brain. Science (New York, N.Y.) 277, 1990-1993.

(70) Wilson, B. A., Ramanathan, A., and Lopez, C. F. (2019) Cardiolipin-Dependent Properties of Model Mitochondrial Membranes from Molecular Simulations. Biophysical Journal 117, 429-444.

(71) Kegel-Gleason, K. B. (2013) Huntingtin interactions with membrane phospholipids: Strategic targets for therapeutic intervention? Journal of Huntington's Disease 2, 239250. 
(72) Beasley, M., Stonebraker, A. R., Hasan, I., Kapp, K. L., Liang, B. J., Agarwal, G., Groover, S., Sedighi, F., and Legleiter, J. (2019) Lipid Membranes Influence the Ability of Small Molecules to Inhibit Huntingtin Fibrillization. Biochemistry 58, 4361-4373.

(73) Tao, M., Pandey, N. K., Barnes, R., Han, S., and Langen, R. (2019) Structure of Membrane-Bound Huntingtin Exon 1 Reveals Membrane Interaction and Aggregation Mechanisms. Structure 27, 1570-1580.e4.

(74) Kameoka, S., Adachi, Y., Okamoto, K., lijima, M., and Sesaki, H. (2020) Phosphatidic Acid and Cardiolipin Coordinate Mitochondrial Dynamics. Trends in Cell Biology 28, 6776.

(75) Joshi, A. S., Thompson, M. N., Fei, N., Ttemann, M. H., and Greenberg, M. L. (2012) Cardiolipin and mitochondrial phosphatidylethanolamine have overlapping functions in mitochondrial fusion in Saccharomyces cerevisiae. Journal of Biological Chemistry 287, 17589-17597.

(76) Frohman, M. A. (2015) Role of mitochondrial lipids in guiding fission and fusion. Journal of Molecular Medicine 93, 263-269.

(77) Sorice, M., Manganelli, V., Matarrese, P., Tinari, A., Misasi, R., Malorni, W., and Garofalo, T. (2009) Cardiolipin-enriched raft-like microdomains are essential activating platforms for apoptotic signals on mitochondria. FEBS Letters 583, 2447-2450.

(78) Ghio, S., Camilleri, A., Caruana, M., Ruf, V. C., Schmidt, F., Leonov, A., Ryazanov, S., Griesinger, C., Cauchi, R. J., Kamp, F., Giese, A., and Vassallo, N. (2019) Cardiolipin Promotes Pore-Forming Activity of Alpha-Synuclein Oligomers in Mitochondrial Membranes.

(79) Nakamura, K., Nemani, V. M., Azarbal, F., Skibinski, G., Levy, J. M., Egami, K., Munishkina, L., Zhang, J., Gardner, B., Wakabayashi, J., Sesaki, H., Cheng, Y., Finkbeiner, S., Nussbaum, R. L., Masliah, E., and Edwards, R. H. (2011) Direct membrane association drives mitochondrial fission by the Parkinson disease-associated protein a-synuclein. Journal of Biological Chemistry 286, 20710-20726.

(80) Kegel, K. B., Sapp, E., Alexander, J., Valencia, A., Reeves, P., Li, X., Masso, N., Sobin, L., Aronin, N., and DiFiglia, M. (2009) Polyglutamine expansion in huntingtin alters its interaction with phospholipids. Journal of Neurochemistry 110, 1585-1597.

(81) Chaibva, M., Burke, K. A., and Legleiter, J. (2014) Curvature enhances binding and aggregation of huntingtin at lipid membranes. Biochemistry 53, 2355-2365.

(82) Beltrán-Heredia, E., Tsai, F. C., Salinas-Almaguer, S., Cao, F. J., Bassereau, P., and Monroy, F. (2019) Membrane curvature induces cardiolipin sorting. Communications Biology 2, 1-7. 
(83) Elías-Wolff, F., LindénSweden., M., Lyubartsev, A. P., and Brandt, E. G. (2019) Curvature sensing by cardiolipin in simulated buckled membranes. Soft Matter 15, 792802.

(84) Pellman, J. J., Hamilton, J., Brustovetsky, T., and Brustovetsky, N. (2015) Ca2+ handling in isolated brain mitochondria and cultured neurons derived from the YAC128 mouse model of Huntington's disease. Journal of Neurochemistry 134, 652-667.

(85) Hamilton, J., Pellman, J. J., Brustovetsky, T., Harris, R. A., and Brustovetsky, N. (2015) Oxidative metabolism in YAC128 mouse model of Huntington's disease. Human Molecular Genetics 24, 4862-4878.

(86) Hamilton, J., Brustovetsky, T., and Brustovetsky, N. (2019) Mutant huntingtin fails to directly impair brain mitochondria. Journal of Neurochemistry 151, 716-731.

(87) Hamilton, J., Brustovetsky, T., Khanna, R., and Brustovetsky, N. (2020) Mutant huntingtin does not cross the mitochondrial outer membrane. Human Molecular Genetics 29, 2962-2975.

(88) Chu, C. T., Ji, J., Dagda, R. K., Jiang, J. F., Tyurina, Y. Y., Kapralov, A. A., Tyurin, V. A., Yanamala, N., Shrivastava, I. H., Mohammadyani, D., Qiang Wang, K. Z., Zhu, J., Klein-Seetharaman, J., Balasubramanian, K., Amoscato, A. A., Borisenko, G., Huang, Z., Gusdon, A. M., Cheikhi, A., Steer, E. K., Wang, R., Baty, C., Watkins, S., Bahar, I., Bayir, H., and Kagan, V. E. (2013) Cardiolipin externalization to the outer mitochondrial membrane acts as an elimination signal for mitophagy in neuronal cells. Nature Cell Biology 15, 1197-1205.

(89) Kagan, V. E., Jiang, J., Huang, Z., Tyurina, Y. Y., Desbourdes, C., Cottet-Rousselle, C., Dar, H. H., Verma, M., Tyurin, V. A., Kapralov, A. A., Cheikhi, A., Mao, G., Stolz, D., St Croix, C. M., Watkins, S., Shen, Z., Li, Y., Greenberg, M. L., Tokarska-Schlattner, M., Boissan, M., Lacombe, M. L., Epand, R. M., Chu, C. T., Mallampalli, R. K., Bayir, H., and Schlattner, U. (2016) NDPK-D (NM23-H4)-mediated externalization of cardiolipin enables elimination of depolarized mitochondria by mitophagy. Cell Death and Differentiation 23, $1140-1151$.

(90) Mejia, E. M., and Hatch, G. M. (2016) Mitochondrial phospholipids: role in mitochondrial function. Journal of Bioenergetics and Biomembranes 48, 99-112.

(91) Ugarte-Uribe, B., Müller, H. M., Otsuki, M., Nickel, W., and García-Sáez, A. J. (2014) Dynamin-related protein 1 (Drp1) promotes structural intermediates of membrane division. Journal of Biological Chemistry 289, 30645-30656.

(92) Macdonald, P. J., Stepanyants, N., Mehrotra, N., Mears, J. A., Qi, X., Sesaki, H., and Ramachandran, R. (2014) A dimeric equilibrium intermediate nucleates Drp1 reassembly on mitochondrial membranes for fission. Molecular Biology of the Cell 25, 1905-1915. 


\section{Oxidation promotes distinct huntingtin aggregates in the presence and absence of membranes}

Huntington's disease is a fatal neurological disorder caused by expanded polyglutamine (polyQ) domain within the first exon (exon1) of huntingtin (htt) protein. PolyQ expansion leads to the formation of a variety of aggregates including globular oligomers, $\beta$-sheet rich fibrils and other amorphous species. Htt aggregation is also influenced by the preceding $17 \mathrm{~N}$-terminal amino acids (Nt17) of htt-exon1. Nt17 also modulates the binding of htt to membranes by forming amphipathic $\alpha$-helix. Many amino acids within Nt17 are susceptible to posttranslational modifications (PTMs), including the two methionine residues that are prone to oxidation. We investigated the impact of oxidation within Nt17 on htt aggregation both in the presence and absence of lipid membranes made from total brain lipid extract (TBLE). We studied this using two experimental systems: htt-exon1 mimic peptide $\left(\mathrm{N} 17-\mathrm{Q}_{35}-\mathrm{P}_{10}\right)$ and full length $\mathrm{htt}-\mathrm{exon} 1$ with 46 glutamine repeats (htt-exon1(46Q)). N17-Q35-P10 exposed to hydrogen peroxide $\left(\mathrm{H}_{2} \mathrm{O}_{2}\right)$ treatment showed reduced fibril formation in an oxidant-dose dependent manner as revealed by Thioflavin $\mathrm{T}$ (ThT) assay. Similarly, htt-exon1(46Q) also showed progressive reduction in fibrillization with increasing $\mathrm{H}_{2} \mathrm{O}_{2}$ as assessed by Atomic Force Microscopy (AFM). Specifically, shorter fibrils and more oligomers were formed at high oxidant concentrations. Upon addition of lipid vesicles, fibrillization was generally hindered in both experimental systems even before the addition of $\mathrm{H}_{2} \mathrm{O}_{2}$. Combined effects of $\mathrm{H}_{2} \mathrm{O}_{2}$ treatment and lipid presence showed similar trend as was observed in the absence of lipids. That is, oligomerization was promoted at the expense of fibril elongation. In addition, the aggregation of both oxidized and unoxidized htt-exon1 was 
monitored on supported lipid bilayers by in situ AFM. The supported lipid bilayers were formed from both oxidized and unoxidized TBLE. In both systems without $\mathrm{H}_{2} \mathrm{O}_{2}$, that is, pre-oxidized and unoxidized bilayers, granular htt aggregates that grew with time were formed. However, in the presence of $\mathrm{H}_{2} \mathrm{O}_{2}$, htt granular aggregates accumulated on the bilayer in distinct plateau-like regions. Collectively, these observations suggest that oxidation of methionine residues within Nt17 plays a crucial role in both the aggregation of htt and its ability to bind lipid membranes.

\subsection{Introduction: Nt17 has posttranslational modification sites and modulates htt aggregation and organelles interaction}

Huntington's disease is a genetic neurological disorder caused by elongation of a polyglutamine (polyQ) stretch within the huntingtin (htt) protein. ${ }^{1}$ PolyQ expansion beyond a critical threshold $(\sim 35)$ triggers htt aggregation into proteinaceous inclusions within cells. ${ }^{2}$ Beyond inclusions, htt forms a variety of other aggregate species, ranging from small oligomers to $\beta$-sheet-rich fibrils. ${ }^{3-6}$ As a consequence, htt aggregation results in a heterogeneous mixture of distinct aggregates. While much effort has been targeted to elucidating specific toxic aggregates, toxic gain of function has been attributed to

oligomers, fibrils, and inclusions. ${ }^{7-12}$ In particular, htt aggregates damage membranous structures throughout the cell, including the $\mathrm{ER},{ }^{12,13}$ mitochondria, ${ }^{14,15}$ and nuclear membranes. ${ }^{16}$ As a result, understanding the aggregation process and the resulting complex mixture of aggregates may be key in unraveling multifaceted toxic profile of HD.

Beyond expansion, amino acid sequences directly flanking the polyQ domain influence aggregation. ${ }^{17-19}$ The first $17 \mathrm{~N}$-terminal residues (Nt17) that directly precede the 
polyQ domain promote fibrillization via oligomeric intermediates ${ }^{17,20}$. This is facilitated by Nt17's ability to form an amphipathic $\alpha$-helix that facilitates the formation of oligomers. ${ }^{20,21}$ Nt17 peptides form a variety of dimers, trimers, and tetramers that arrange in an antiparallel manner. ${ }^{22,23}$ The resulting $\alpha$-helix-rich oligomers bring polyQ domains from different htt proteins together to accelerate the nucleation process. ${ }^{17,20,24}$

Approximately half of $\mathrm{htt}$ present in the cytoplasm is associated with membranes. ${ }^{25-27}$ The ability of Nt17 to form an amphipathic $\alpha$-helix also facilitates httexon's ability to bind lipid membranes. ${ }^{21,28} \mathrm{Nt17}$ is involved in targeting htt to the autophagic vacuoles, and ER, mitochondrial, and Golgi membranes. ${ }^{29,30} \mathrm{~A}$ variety of toxic processes may encompass htt/lipid interactions. Htt association with organelle membranes is linked with disrupted trafficking, ${ }^{31}$ synaptic degeneration, ${ }^{32}$ and neuronal cell death. ${ }^{33}$ Lipid membranes are also susceptible to htt-induced disruption and permeabilization. ${ }^{14,34-36}$ The direct interaction facilitated by $\mathrm{Nt17}$ of htt-exon1 with intracellular and subcellular membranes further adds to the complexity of the aggregation process. Lipid membrane can either increase ${ }^{37}$ or decrease ${ }^{38}$ fibrillization, depending on the physicochemical properties of the bilayer based on lipid composition. ${ }^{39-41}$

Both the ability of Nt17 to promote htt aggregation and bind lipid membranes is modulated by post translational modifications (PTMs) within Nt17.42,43 Interestingly, Nt17 can undergo a variety of post-translational modifications (PTMs), including oxidation, ${ }^{44}$ phosphorylation, ${ }^{45,46}$ acetylation, ${ }^{47}$ SUMOylation ${ }^{48}$ and ubiquitination. ${ }^{49}$ Many of these PTMs impact oligomer and fibril formation, ${ }^{42,43,50}$ potentially by altering the stability of the a-helix-rich oligomeric intermediates. Oxidative stress is caused by an imbalance between the production of reactive oxidative species (ROS) and antioxidant cellular 
defenses. At high ROS levels, htt localization is shifted from cytoplasmic membranes such as the ER towards nuclear entry, ${ }^{44}$ suggesting that oxidation alters the affinity of $\mathrm{Nt17}$ for the ER. In this regard, the Nt17 may function as a ROS sensor, as it contains two methionine residues (M1 and M8) susceptible to oxidation. ${ }^{44}$ Methionine (M8) plays a crucial role in stabilizing the Nt17 helical conformation, and modification of this residue triggers htt -ER release, resulting in cellular toxicity without the formation of larger htt ${\text { aggregates. }{ }^{30} \text { Similarly, other studies have observed reduced or inhibited }}^{19,51,52}$ aggregation of htt-exon1 mimicking peptides that were oxidized.

Here, we investigate how oxidative environments, produced by $\mathrm{H}_{2} \mathrm{O}_{2}$, influence httexon1 aggregation in the presence and absence of model total brain lipid extract (TBLE) lipid bilayers. TBLE was chosen as the model lipid system as it is comprised of a physiologically relevant ratio of lipids, i.e., acidic and neutral phospholipids, gangliosides, cholesterol, sphingolipids, and isoprenoids. In the absence of lipid, exposure to hydrogen peroxide suppressed htt fibrillization in a dose-dependent manner. This fibril suppression was accompanied with an increase in the oligomer population accompanied by a slight shift to larger oligomers. With the introduction of TBLE vesicles, htt aggregation was further reduced. Oxidation modified htt-induced morphological changes on the surface of the TBLE bilayers.

\subsection{Methods}

3.2.1 Preparation of synthetic peptides. A synthetic htt-exon1 mimic peptide (Anaspec) consisting of Nt17, a polyQ stretch of 35 residues, and a polyproline stretch of 10 residues (referred to as Nt17-Q35-P10) was solubilized based on previously described protocols. ${ }^{53}$ The peptide was initially treated with a 1:1 mixture of Hexafluoroisopropanol 
(HFIP) and Trifluoroacetic acid (TFA). The solvent was then removed under vacuum in a vacufuge (Eppendorf). The resulting dried films were stored at $-20^{\circ} \mathrm{C}$ until needed. Prior to any experiment, the peptide film was reconstituted in dimethyl sulfoxide (DMSO) to a concentration of $4000 \mu \mathrm{M}$. The DMSO stock solution was then diluted $200 \times$ in aggregation buffer [50 mM Tris- $\mathrm{HCl}(\mathrm{pH} 7)$ and $150 \mathrm{mM} \mathrm{NaCl}$ to achieve a final peptide concentration of $20 \mu \mathrm{M}$ with $0.5 \%$ DMSO.

3.2.2 Purification of GST-htt exon1 fusion protein: Gluthathione Stransferase(GST)-htt-exon1(46Q) fusion proteins was expressed in Escherichia coli purified as previously described. ${ }^{3}$ Briefly, after induction with isopropyl thiogalactopyranoside (IPTG) for $4 \mathrm{~h}$ at $30^{\circ} \mathrm{C}$, the cells were lysed by a combination of lysozyme and probe sonication. The GST-htt fusion protein was purified from the lysate using affinity liquid chromatography (Bio-Rad LPLC, GST-affinity column). Relevant protein fractions and purity were determined by SDS-PAGE. Prior to every experiment, GST-htt-exon1(46Q) fusion proteins were subjected to high spin centrifugation $\left(22,000 \times \mathrm{g}, 4^{\circ} \mathrm{C}, 30 \mathrm{~min}\right)$ to remove pre-existing aggregates, and protein concentration was measured with a Bradford assay. Cleavage of GST was facilitated by incubation with factor Xa for 90 min on ice (New England Biolabs). All experiments were carried out in aggregation buffer [50 mM Tris- $\mathrm{HCl}(\mathrm{pH} 7)$ and $150 \mathrm{mM} \mathrm{NaCl}]$.

3.2.3 Preparation of lipid vesicles: Lipid vesicles were prepared as previously described ${ }^{54}$ with some modifications. Total brain lipid extract (TBLE; Avanti Polar Lipids, Alabaster, AL) were dissolved in chloroform, aliquoted into smaller samples, placed in a vaccufuge to remove the choloroform, and the dried films were stored at $-20^{\circ} \mathrm{C}$ until needed. For experiments using pre-oxidized lipid, TBLE samples were oxidized by 
hydrating the lipid films with $300 \mu \mathrm{L}$ of $3 \%(\mathrm{w} / \mathrm{v}) \mathrm{H}_{2} \mathrm{O}_{2}$, incubated in a Thermomixer at 60 ${ }^{\circ} \mathrm{C}$ and $1400 \mathrm{rpm}$ for $3 \mathrm{~h}$, aliquoted into smaller samples, dried in a vacufuge, and stored at $-20{ }^{\circ} \mathrm{C}$. Unoxidized and oxidized TBLE films were reconstituted in the aggregation buffer to obtain $1 \mathrm{mg} / \mathrm{ml}$ solutions with vigorous mixing at $60^{\circ} \mathrm{C}$ for $30 \mathrm{~min}$. Lipid vesicles were made by subjecting films to 10 freeze-thaw cycles followed by bath sonication for 1 h. ThT and incubation experiments were carried out by adding $30 \mu \mathrm{L}$ of the lipid stock solution to protein samples (synthetic peptide or htt-exon1(46Q)) to achieve a final concentration of $0.3 \mathrm{mg} / \mathrm{mL}$ of lipid and $20 \mu \mathrm{M}$ protein.

3.2.4 Thioflavin $T$ (ThT) assay: Monitoring fibril formation was achieved with thioflavin T (ThT; Sigma-Aldrich, St. Louis, MO) assays. ThT ssays were carried out in a Costar 96-well black clear flat bottom plate.using a SpectraMax M2 plate reader. All experiments were performed at, $37^{\circ} \mathrm{C}$ for $10 \mathrm{~h}$ with reading every $5 \mathrm{~min}$. The excitation wavelength was $440 \mathrm{~nm}$, and the emission wavelength was $484 \mathrm{~nm}$. All conditions were performed in triplicate on any give plate, and a minimum of three independent plates were run for all experiments. The final concentration of the Nt17-Q35P10 peptide was $20 \mu \mathrm{M}$ in all wells, and the final concentrations of $\mathrm{H}_{2} \mathrm{O}_{2}$ were $0.02,0.1$, $0.25,0.5,10$ or $20 \mathrm{mM}$ to achieve the $1 \mathrm{x}, 5 \mathrm{x}, 12.5 \mathrm{x}, 25 \mathrm{x}, 500 \mathrm{x}$, and $1000 \mathrm{x}$ relative to the peptide concentration conditions. Similar assays were performed in the presence of TBLE vesicles $(0.3 \mathrm{mg} / \mathrm{ml}$ final concentration). The ThT concentration per well was $60 \mu \mathrm{M}$.

3.2.5 Polydiacetylene (PDA) Vesicle Binding Assay: 10,12-Tricosadiynoic acid (GFS Chemicals, Columbus, OH) and TBLE dissolved at a 2:3 molar ratio in a 1:4 ethanol/chloroform solution. The solution was evaporated off under a stream of $\mathrm{N}_{2}$, and 
the resulting film was dissolved with aggregation buffer at $70{ }^{\circ} \mathrm{C}$. The solution was sonicated for 10 min intervals at $100 \mathrm{~W}$ using a sonic dismembrator (FisherSci) to achieve an opaque solution that was stored overnight at $4{ }^{\circ} \mathrm{C}$ to allow for the formation of mixed vesicles. The PDA was then polymerized by irradiation at $254 \mathrm{~nm}$ for $10 \mathrm{~min}$ at room temperature with constant stirring. Polymerization resulted in a blue solution. The colorimetric response was monitored by ultraviolet-visible (UV-vis) spectroscopy to quantify the binding of htt-exon1(46Q) to vesicles with a SpectraMax M2 microplate reader (Molecular Devices) in a 96-well plate format. Each plate contained negative controls (neat buffer $A$ ) and a positive control (final concentration of $100 \mu \mathrm{M} \mathrm{NaOH}$ ). The $\mathrm{NaOH}$ control was used to standardize PDA results across experiments. ${ }^{55}$ Readings were taken every $5 \mathrm{~min}$ for $18 \mathrm{~h}$ at $25^{\circ} \mathrm{C}$ by measuring the blue absorbance (640 nm, Ablue) and the red absorbance $\left(500 \mathrm{~nm}, A_{\mathrm{red}}\right)$. The \% colorimetric response (\%CR) was then determined by the following equation:

$$
\% C R=\frac{P B_{0}-P B_{1}}{P B_{0}} \times 100
$$

where PB is defined as $A_{\text {blue }} /\left(A_{\text {blue }}+A_{\text {red }}\right)$. $\mathrm{PB}_{0}$ is determined from the negative control, and $\mathrm{PB}_{1}$ is obtained from each experimental condition. ${ }^{56,57}$

3.2.6 Atomic Force Microscopy (AFM): AFM experiments were performed with a Nanoscope V Multimode atomic force microscope (Veeco, Santa Barbara, CA) equipped with a closed-loop vertical engage J-scanner. For ex situ AFM, incubations of httexon1(46Q) $(20 \mu \mathrm{M})$ were mixed with $\mathrm{H}_{2} \mathrm{O}_{2}$ to achieved various ratios of htt-exon1(46Q): $\mathrm{H}_{2} \mathrm{O}_{2}$ 1:5 or $5 \mathrm{x}$, (1:50 or $50 x, 1: 200$ or $200 x$ and 1:1000 or $\left.1000 x\right)$. A duplicate of each 
system was also mixed with TBLE vesicles to a final lipid concentration of $(0.30 \mathrm{mg} / \mathrm{ml}$ or $\sim 400 \mu \mathrm{M})$. A third system involves the mixing of unoxidized htt-exon1(46Q) with oxidized TBLE vesicles. All systems were maintained at $37^{\circ} \mathrm{C}$ and aliquots of the incubations drawn at various time points were deposited on freshly cleaved mica (Ted Pella Inc., Redding, CA) and allowed to rest for $75 \mathrm{~s}$. The mica was then washed with $200 \mu \mathrm{L}$ of ultrapure water and dried with a gentle stream of air. Dried samples were measured by ex situ AFM with oxide-sharpened silicon cantilever with a nominal spring constant of 40 $\mathrm{N} / \mathrm{m}$ and resonance frequency of $\sim 300 \mathrm{kHz}$.

For in situ AFM experiments, a fluid cell equipped with a silicon nitride cantilever (nominal spring constant of $0.1 \mathrm{~N} / \mathrm{m}$ ) was used. The drive frequency ranged between $7.5-$ $9.5 \mathrm{kHz}$, and scan rate was maintained between $1.5-2 \mathrm{~Hz}$. Initially, background scans of freshly cleaved mica under neat buffer $(20 \mu \mathrm{L})$ were taken to ensure the cleanliness of the fluid cell. Next, an equal volume of the prepared $1 \mathrm{mg} / \mathrm{ml}$ of TBLE or pre-oxidized TBLE vesicles were introduced to a final concentration of $0.5 \mathrm{mg} / \mathrm{ml}$ within the fluid cell. Bilayer formation on mica via vesicle fusion was observed by continuous imaging. Once sufficient bilayer formed, cleaved htt-exon1(46Q) (or htt-exon1(46Q) that had been cleaved and incubated with $1000 \mathrm{x}_{2} \mathrm{O}_{2}$ on ice) was injected to achieve a final protein concentration of $10 \mu \mathrm{M}$ and lipid concentration of $0.25 \mathrm{mg} / \mathrm{ml}$ in the fluid cell. After protein injection, images were collected continuously for up to $160 \mathrm{~min}$. For both ex situ and in situ AFM experiments, images were processed and analyzed with a combination of WSxM ${ }^{58}$ and Matlab equipped with the image processing toolbox (MathWorks, Natick, MA) as previously described. ${ }^{59}$ 


\subsection{Results}

3.3.1 Oxidation suppresses htt fibrilization. To establish if the presence of $\mathrm{H}_{2} \mathrm{O}_{2}$ would alter htt aggregation, the rate of fibril formation of a synthetic htt-mimic peptide containing Nt17, a 35 residue long polyQ domain, and a 10 residue polyP domain $\left(\mathrm{Nt}^{17} \mathrm{Q}_{35} \mathrm{P}_{10}\right.$ ) was investigated (Fig. 3.1). $20 \mu \mathrm{M}$ of $\mathrm{Nt}^{17} \mathrm{Q}_{35} \mathrm{P}_{10}$ was exposed to various concentrations of $\mathrm{H}_{2} \mathrm{O}_{2}$ to achieve peptide: $\mathrm{H}_{2} \mathrm{O}_{2}$ ratios ranging from 1:1 to 1:1000.

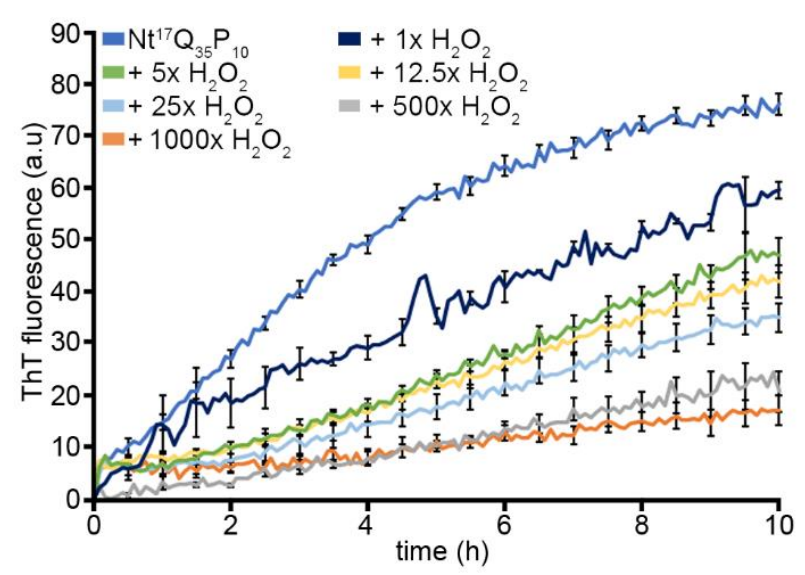

Figure 3.1: The impact of $\mathrm{H}_{2} \mathrm{O}_{2}$ on $\mathrm{Nt}^{17} \mathrm{Q}_{35} \mathrm{P}_{10}$ aggregation as assessed by a ThT assay. $\mathrm{Nt}^{17} \mathrm{Q}_{35} \mathrm{P}_{10}$ was incubated at $20 \mu \mathrm{M}$ without $\mathrm{H}_{2} \mathrm{O}_{2}$ (control) and with $\mathrm{H}_{2} \mathrm{O}_{2}$ at molar ratios of $1 x, 5 x, 12.5 x, 25 x, 500 x$, and $1000 x$ that of the peptide.

Aggregation was monitored using a ThT assay, which detects $\beta$-sheet formation associated with fibrils. ${ }^{60}$ Based on this assay, treatment with $\mathrm{H}_{2} \mathrm{O}_{2}$ reduced fibril formation in a dose dependent manner (Fig. 3.1). With as little as $1 \mathrm{x} \mathrm{H}_{2} \mathrm{O}_{2}$, the ThT signal was significantly reduced by $\sim 25 \%$. Increasing the dose of $\mathrm{H}_{2} \mathrm{O}_{2}$ to $5 x-25 x$ further reduced fibril formation by $\sim 40-60 \%$ depending on the exact dose. With large doses (500x and 1000x) of $\mathrm{H}_{2} \mathrm{O}_{2}$, the ThT signal was reduced by $\sim 75-80 \%$ compared with control. 
While ThT assays indicate that the oxidative environment provided by $\mathrm{H}_{2} \mathrm{O}_{2}$ reduces fibril formation, these assays do not provide direct information on aggregate morphologies and species beyond fibrils. As a result, AFM assays were performed using a GST/mutant htt-exon1 fusion protein containing a 46 residue long polyQ domain that 46Q (htt-exon1(46Q)). Incubations of htt-exon1(46Q) $\left(20 \mu \mathrm{M}, 37^{\circ} \mathrm{C}\right)$ were treated with $\mathrm{H}_{2} \mathrm{O}_{2}$ at various protein to peroxide ratios ranging from 1:5 to 1:1000. Control incubations of htt-exon1(46Q) in which no peroxide was added were also prepared. Aliquots were taken from each incubation at 1, 3, 5, and $8 \mathrm{~h}$ and deposited onto mica for AFM imaging and analysis (Fig. 3.2). Fibrils were present and increased in occurrence with time under all conditions; however, the extent of fibrillization appeared reduced with increasing dose of $\mathrm{H}_{2} \mathrm{O}_{2}$ (Fig. 3.2A). In the absence of $\mathrm{H}_{2} \mathrm{O}_{2}$, fibrils were observed within $1 \mathrm{~h}$ of incubation. With time, fibrils appeared longer and were predominately clustered together into large bundles. Similar patterns of fibril formation were observed with $5 x$ and $50 x$ treatments of $\mathrm{H}_{2} \mathrm{O}_{2}$; although, bundling of fibrils was less prominent compared with control. When $\mathrm{H}_{2} \mathrm{O}_{2}$ treatments were increased to $200 x$ and $1000 x$, fibrils were still observed, but large bundles were mainly absent.

To quantify this observation, all AFM images were analyzed using a combination of features in WSxM ${ }^{58}$ and automated scripts that were written in Matlab. WSxM was used to measure fibril contour lengths. Automated Matlab scripts were written to identify individual aggregates and measures a variety of other morphological features. For these automated measurements, fibrils were defined as features with a height greater than 2.5 $\mathrm{nm}$ and occupying an area in the image of at least $10,000 \mathrm{~nm}^{2}$. The identified features were manually inspected to exclude non-fibrillar structures (large amorphous structures) 
from any further analysis. A greater number of fibrils per $\mu \mathrm{m}^{2}$ (based on the above criteria) was observed with increasing $\mathrm{H}_{2} \mathrm{O}_{2}$ dose at each time point (Fig. 3.2B); however, this raw count of fibrils does not accurately reflect the fibril load under the different conditions. This is due to fibrils under different conditions having distinct morphological features. That is, the proclivity of fibrils to bundle in the absence of or low doses of $\mathrm{H}_{2} \mathrm{O}_{2}$ created inaccuracies in fibrils counts. For the control and $5 \mathrm{x} \mathrm{H}_{2} \mathrm{O}_{2}$ incubations, the number of fibrils per area fluctuates, but stays relatively consistent throughout the experiment. With $50 x \mathrm{H}_{2} \mathrm{O}_{2}$, the number of fibrils initially increases but drops at later time points. This coincides with the appearance of fibril bundles. With $200 x$ and $1000 \times \mathrm{H}_{2} \mathrm{O}_{2}$ treatments, the number of fibrils steadily increased. As these conditions did not display excessive bundle formation at later time points, the fibril count does not display a drop. Additionally, the contour length of fibrils drastically decreased with increasing dose of $\mathrm{H}_{2} \mathrm{O}_{2}$ (Fig. 3.2C). Initially, the fibrils observed after $1 \mathrm{~h}$ of incubation were similar in contour length under all conditions; however, fibril elongation was severely stunted with increasing $\mathrm{H}_{2} \mathrm{O}_{2}$ treatment. The mean fibril length at $8 \mathrm{~h}$ for incubations of htt-exon1(46Q) without $\mathrm{H}_{2} \mathrm{O}_{2}$ or with a $5 x$ dose of $\mathrm{H}_{2} \mathrm{O}_{2}$ were both near $2 \mu \mathrm{m}$; however, fibrils formed in the presence of $200 x$ or $1000 \mathrm{x} \mathrm{H}_{2} \mathrm{O}_{2}$ were on average shorter than $500 \mathrm{~nm}$. Due to these morphological differences across conditions, the average surface area covered by fibrils was determined to obtain a more accurate measure of fibril load across the different conditions (Fig. 3.2D). Largest fibril area formed by the control (htt-exon1(46Q), no oxidation) was normalized and the fibril areas from other conditions were reported relative to the control. Based on this analysis, the extent of htt fibril formation clearly decreases with increasing $\mathrm{H}_{2} \mathrm{O}_{2}$ concentration, consistent with the ThT assay. The large error bars associated with the 
fibril area coverage of the htt-exon1(46Q) control and the $5 \mathrm{x} \mathrm{H}_{2} \mathrm{O}_{2}$ incubations is due to the localization of massive fibril content due to bundling. Collectively, these observations suggest that oxidation of htt-exon1(46Q) interferes strongly with fibril elongation. That is, a similar number of individual fibrils appeared after $1 \mathrm{~h}$ of incubation for all conditions when bundling did not interfere with the accuracy of fibril counts. At high $\mathrm{H}_{2} \mathrm{O}_{2}$ doses (200x and 1000x) the number of fibrils readily increased with time to similar extents, but these fibrils remained short compared to their counterparts without or with low doses of $\mathrm{H}_{2} \mathrm{O}_{2}$.

With the potential for oxidative conditions to interfere with fibril elongation, the height (or thickness) of fibrils under the different conditions was analyzed to determine if oxidation may be impacting the ultrastructure of fibrils. Height profiles were drawn across single fibril strands and fibril bundles (Fig. 3.2E). Due to extensive bundle formation, single strands for incubations without and with low doses of $\mathrm{H}_{2} \mathrm{O}_{2}$ were often identified protruding from bundles. Across all oxidation conditions, no apparent height difference was observed for single strand fibrils. Single fibrils ranging from 5-8 $\mathrm{nm}$ in height were readily observed across all conditions and time points. This suggests that despite differences in the rate of formation and elongation that the fibrils under all conditions likely have a similar polyQ core structure, which has been identified by several ssNMR studies. ${ }^{61,62}$ As expected, fibril bundles were much thicker than single strands, reaching 25-30 nm in height. 


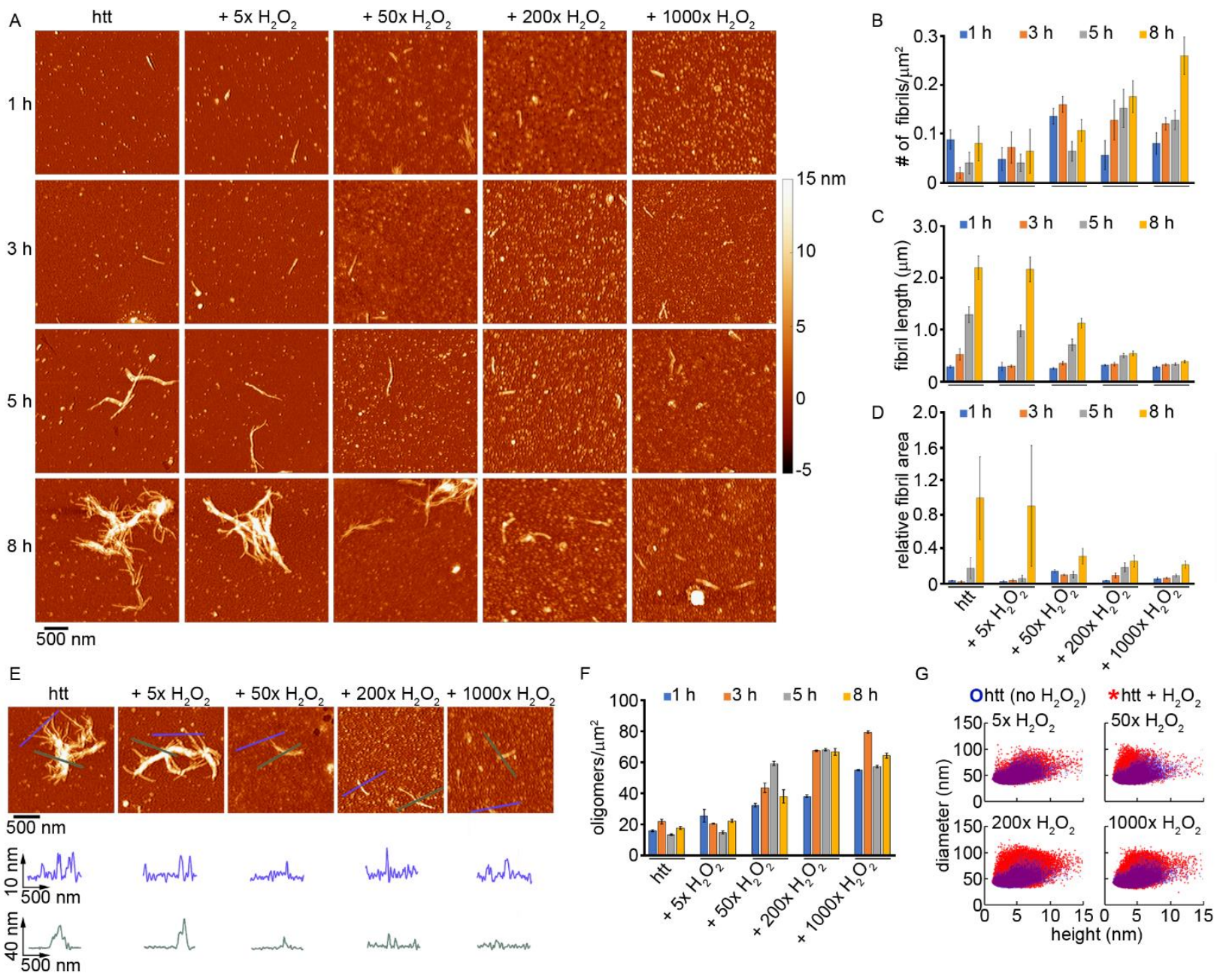

Figure 3.2: Oxidation alters the aggregation of htt. (A) Representative ex situ AFM images of aliquots of htt-exon1(46Q) $(20 \mu \mathrm{M})$ with $\mathrm{H}_{2} \mathrm{O}_{2}$ to achieve various final $\mathrm{H}_{2} \mathrm{O}_{2}$ concentrations of $0,100 \mu \mathrm{M}, 1 \mathrm{mM}, 4 \mathrm{mM}$ and $20 \mathrm{mM}$. Aliquots were sampled at 1,3,5 8 $h$. (B) Analysis of fibril population. Fibrils were counted at the different time points and presented as fibrils/ $\mu^{2}{ }^{2}$. (C) Analysis of fibril length using WSXM. Fibrils were traced to obtain the average fibril length for each condition. (D) Analysis of area covered by fibrils. These values were normalized to the fibril covered by htt control (no oxidation) at $8 \mathrm{~h}$. (E) Height profiles of fibrils. The height profiles of fibril strands and bundles are shown on different height scales. (F) Oligomers population analysis. Oligomers were identified using automated scripts written in matlab. Oligomers are defined as globular features taller than $1.0 \mathrm{~nm}$ and having coverage area between 500-10000 nm². (G) Analysis of oligomers height. Oligomers from all time points were combined per condition and the heights of the combined data is presented. 
3.3.2 Oxidation promotes htt oligomerization. Having established that oxidation suppressed fibrillization, AFM images were next analyzed for potential changes in oligomer formation. Again, using automated scripts in Matlab, oligomers were identified, and morphological features of these oligomers were determined. For this analysis, oligomers were defined as any feature taller than $1.0 \mathrm{~nm}$ with an occupied area of 500$10,000 \mathrm{~nm}^{2}$. Due to the small size of oligomers in the images presented in Fig. 3.2, zoomed in, representative images of oligomers obtained at 1 and $3 \mathrm{~h}$ for all conditions are provided in Fig. 3.3. The population of oligomers observed in incubations of httexon1(46Q) alone or with $5 \times \mathrm{H}_{2} \mathrm{O}_{2}$ were relatively steady with less than 20 oligomers $/ \mu \mathrm{m}^{2}$ at each time point (Fig. 3.2F). Higher oxidation conditions (50x $-1000 x)$ generally increased the oligomer populations. At $50 \mathrm{x} \mathrm{H}_{2} \mathrm{O}_{2}$, htt oligomers increased for up to $5 \mathrm{~h}$ (Fig. 3.2F) with a marked drop off at $8 \mathrm{~h}$. This decrease roughly correlates with an increase in fibril load previously discussed. At $200 \mathrm{x} \mathrm{H}_{2} \mathrm{O}_{2}$, the oligomer population surged at $3 \mathrm{~h}$, resulting in a large, stable population of oligomers at 5 and $8 \mathrm{~h}$. A similar large, stable oligomer population was apparent with $1000 \times \mathrm{H}_{2} \mathrm{O}_{2}$ throughout the incubation. These observations suggest that suppression of fibril elongation due to exposure to increasing concentration of $\mathrm{H}_{2} \mathrm{O}_{2}$ allows for the formation of a larger oligomer population. To determine if this change in oligomer population size could be associated with distinct oligomer types, oligomer morphological features were compared across the different incubation conditions. The height of each oligomer was obtained using the same Matlab script, but by determining the peak pixel of every identified oligomer. The height and diameter of each individual oligomer were used to construct correlation plots (Fig. 3.2G). With a $5 x$ dose of $\mathrm{H}_{2} \mathrm{O}_{2}$, oligomer morphology was indistinguishable from control. 
However, with increasing $\mathrm{H}_{2} \mathrm{O}_{2}$ treatment, the distribution of oligomer morphologies broadens in the correlation plots. Collectively, the presence of higher oxidant concentrations increased the population and size of htt-exon1(46Q) oligomers.
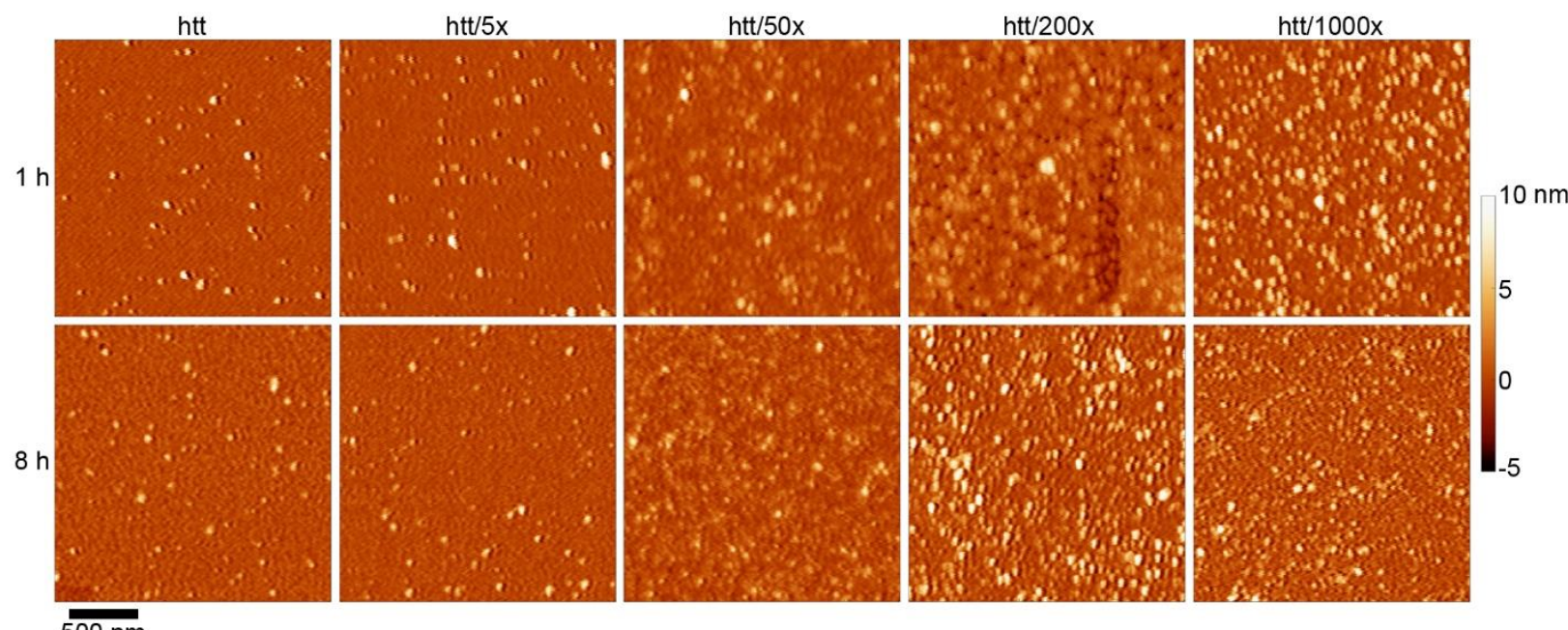

Figure 3.3: Oxidation of htt-exon1(46Q) promoted oligomerization. Zoomed in images at 1 and $3 \mathrm{~h}$ showing increased oligomerization with increase in oxidation. $20 \mu \mathrm{M}$ htt-exon1(46Q) was incubated without and with 0.1, 1, 4 and $20 \mathrm{mM} \mathrm{H}_{2} \mathrm{O}_{2}$.

3.3.3 Oxidation reduces htt fibrilization in the presence of lipids. As a large proportion of htt is associated with lipid membranes in vivo and lipids influence htt aggregation,,$^{37,63-65}$ the impact of oxidative environments on htt aggregation in the presence of TBLE vesicles was determined. The fibrillization of the $\mathrm{Nt}^{17}-\mathrm{Q}_{35}-\mathrm{P}_{10}$ peptide in the presence of TBLE vesicles (1:20 peptide to lipid ratio) and various doses of $\mathrm{H}_{2} \mathrm{O}_{2}$ was monitored using the ThT assay (Fig. 3.4). The aggregation of $\mathrm{Nt}^{17}-\mathrm{Q}_{35}-\mathrm{P}_{10}$ in the absence of TBLE vesicles or $\mathrm{H}_{2} \mathrm{O}_{2}$ was used as a control. Consistent with previous reports, ${ }^{50,66}$ TBLE suppressed fibrillization, as the ThT signal was reduced by $\sim 60 \%$ 
relative to the control. Addition of $\mathrm{H}_{2} \mathrm{O}_{2}$ to $\mathrm{Nt}^{17}{ }^{17} \mathrm{Q}_{35}-\mathrm{P}_{10}$ incubations with TBLE vesicles further reduced fibrillization in a dose dependent manner. The ThT signal associated with the addition of $1 \mathrm{x} \mathrm{H}_{2} \mathrm{O}_{2}$ to the condition with just the addition of TBLE vesicles was reduced by $\sim 50 \%$. $5 x$ and $12.5 \times \mathrm{H}_{2} \mathrm{O}_{2}$ treatments reduced the ThT signal by $\sim 60 \%$. Larger doses of $\mathrm{H}_{2} \mathrm{O}_{2}$ prevented any increase in ThT signal.

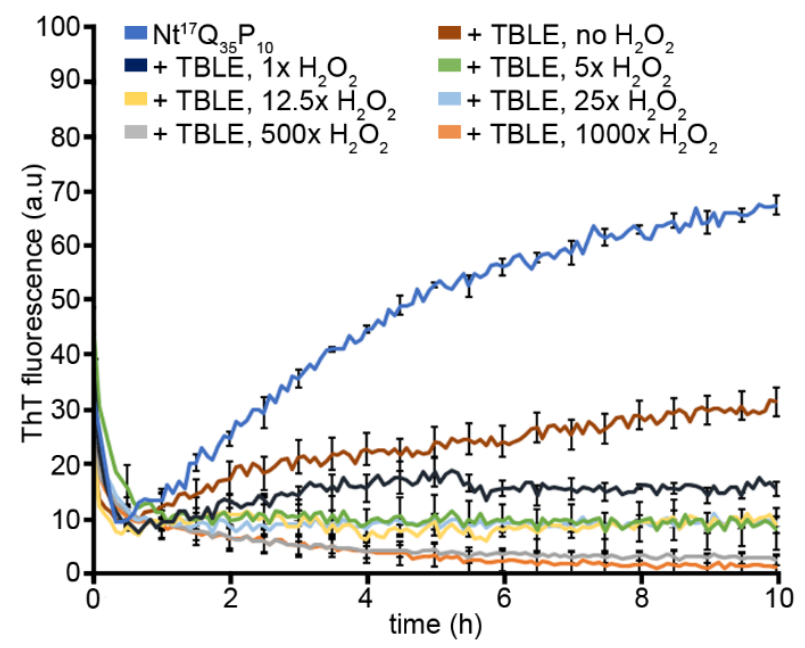

Figure 3.4: ThT analysis of oxidized and unoxidized $\mathrm{Nt}^{17}-\mathrm{Q}_{35}-\mathrm{P}_{10}$ peptide aggregation in the presence of lipid vesicles. Aggregation of $N t^{17}-Q_{35}-P_{10}(20 \mu \mathrm{M})$ monitored in the presence and absence of TBLE vesicles ( 400 $\mu$ M). Samples containing TBLE were further subjected to various oxidation conditions using $\mathrm{H}_{2} \mathrm{O}_{2}$ to achieve final $\mathrm{H}_{2} \mathrm{O}_{2}$ concentrations of $0,20 \mu \mathrm{M}, 100 \mu \mathrm{M}, 250 \mu \mathrm{M}, 500 \mu \mathrm{M}, 10 \mathrm{mM}$, and $20 \mathrm{mM}$.

Due to the presence of two oxidizable methionine residues in $\mathrm{Nt17}, \mathrm{H}_{2} \mathrm{O}_{2}$ could be altering the interaction of htt with the TBLE. Therefore, the impact of $\mathrm{H}_{2} \mathrm{O}_{2}$ on the ability of htt-exon1(46Q) to bind the TBLE vesicles was determined by a polydiacetylene (PDA) lipid binding assay. PDA is a lipid moiety that is readily incorporated with other lipids into vesicles. Photo-cross-linking of the PDA creates vesicles that are sensitive to mechanical perturbations associated with the direct interaction of proteins with the lipids. This sensitivity results in a color change from blue to red that can be monitored 
spectroscopically as a colorimetric response (CR). Here, TBLE/PDA lipid vesicles were exposed to htt-exon1(46Q) in the presence of various doses of $\mathrm{H}_{2} \mathrm{O}_{2} . \mathrm{H}_{2} \mathrm{O}_{2}$ did not invoke a CR response in the PDA assay in control experiments, and these controls were incorporated into the calculation of the CR based on Eq. 1. Based on the PDA assay, $\mathrm{H}_{2} \mathrm{O}_{2}$ did not alter the extent of interaction of htt with TBLE lipid vesicles; although, there appears to be a slight lag in the htt/lipid interaction with treatment with $1000 \mathrm{x} \mathrm{H}_{2} \mathrm{O}_{2}$ (Fig. 3.5).

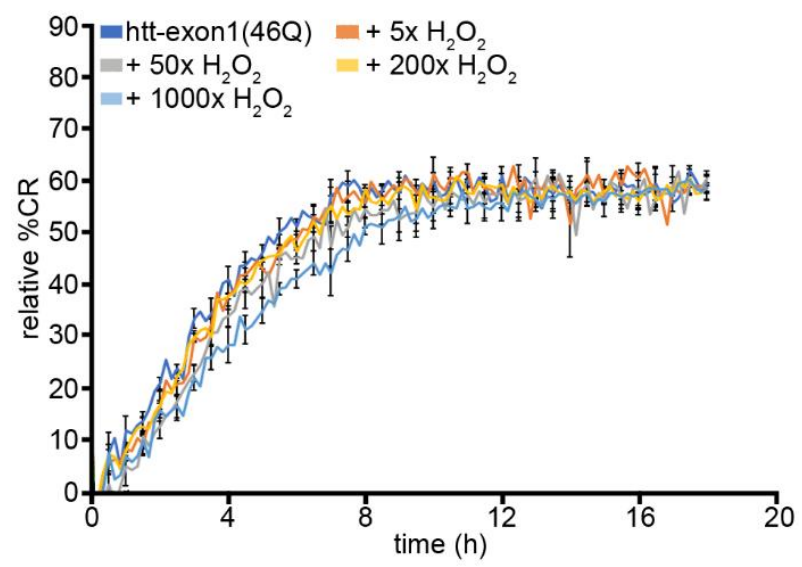

Figure 3.5: PDA/TBLE lipid binding assay of htt-exon1(46Q) exposed to no or various $\mathrm{H}_{2} \mathrm{O}_{2}$ concentrations. The colorimetric response was calculated as a function of time upon exposure to $20 \mu \mathrm{M}$ htt-exon1(46Q) (deep blue) incubated at $25{ }^{\circ} \mathrm{C}$ and in the presence of $0.1 \mathrm{mM}$ (orange), $1 \mathrm{mM}$ (grey), $4 \mathrm{mM}$ (light blue), $20 \mathrm{mM} \mathrm{H}_{2} \mathrm{O}_{2}$ (yellow).

Similar to experiments without lipid, the impact of oxidation on htt aggregation in the presence of lipids was further investigated using AFM. Incubations of htt-exon1(46Q) $\left(20 \mu \mathrm{M}, 37^{\circ} \mathrm{C}\right.$ ) with TBLE vesicles (protein to lipid ratio of $1: 20$ ) were treated with $\mathrm{H}_{2} \mathrm{O}_{2}$ at various protein to peroxide ratios ranging from $1: 5$ to $1: 1000$. In addition to control incubations of htt-exon1(46Q) with TBLE vesicles in which no peroxide was added, a control incubation of htt-exon1(46Q) with pre-oxidized TBLE vesicles was also performed. 

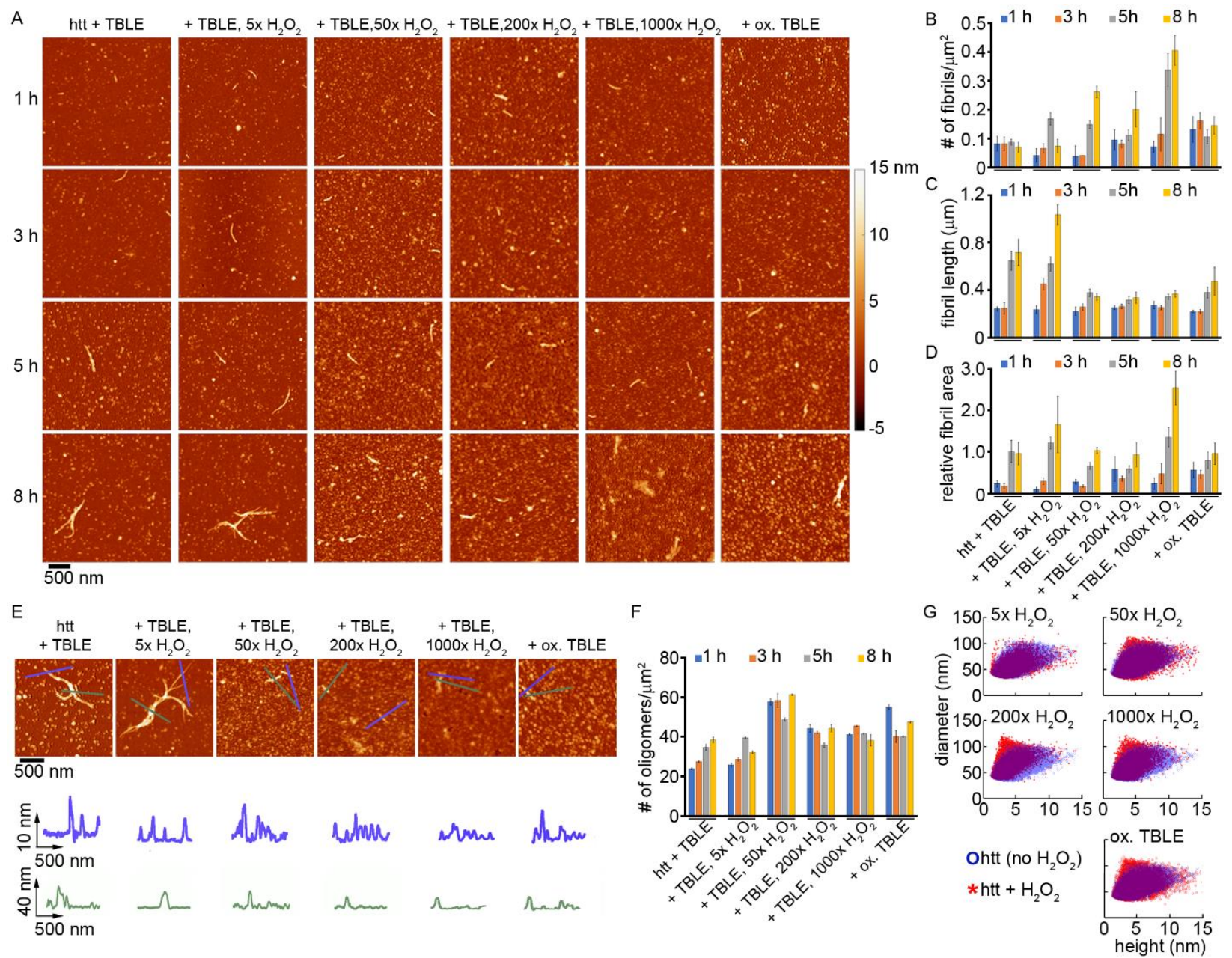

Figure 3.6: Presence of lipid vesicles alter the aggregation of oxidized htt. Unoxidized TBLE vesicles were incubated with htt-exon1(46Q) in the presence of final $\mathrm{H}_{2} \mathrm{O}_{2}$ concentrations of $0,100 \mu \mathrm{M}, 1 \mathrm{mM}, 4 \mathrm{mM}$ and $20 \mathrm{mM}$. Oxidized TBLE vesicles were incubated with htt-exon1(46Q) with no additional $\mathrm{H}_{2} \mathrm{O}_{2}$. Aliquots were sampled at 1,3,5 8 h. (A) Representative ex situ AFM images of all experimental conditions. (B) Analysis of fibril population. Fibrils were counted at the different time points and presented as fibrils $/ \mu m^{2}$. (C) Analysis of fibril length using WSXM. Fibrils were traced to obtain the average fibril length for each condition. (D) Analysis of area covered by fibrils. These values were normalized to the fibril covered by htt incubated with unoxidized TBLE (control, no oxidation) at $8 \mathrm{~h}$. (E) Height profiles of fibrils. The height profiles of fibril strands and bundles are shown on different height scales. (F) Oligomers population analysis. Oligomers were identified using automated scripts written in matlab. Oligomers are defined as globular features taller than $1.0 \mathrm{~nm}$ and covering 500-10000 $\mathrm{nm}^{2}$. (G) Analysis of oligomers height. Oligomers from all time points were combined per condition and the heights of the combined data is presented. 
That is, the htt remained unmodified. For this control, TBLE was treated with the same concentration of $\mathrm{H}_{2} \mathrm{O}_{2}$ used for the $1000 x$ condition for $3 \mathrm{~h}$. Residual $\mathrm{H}_{2} \mathrm{O}_{2}$ was removed via lyophilization, and the lipid was reconstituted for these incubations. Aliquots were taken from each incubation at 1, 3, 5, and $8 \mathrm{~h}$ for AFM analysis (Fig. 3.6). Comparing the AFM images of unoxidized htt-exon1(46Q) without (Fig. 3.2A) and with vesicles (Fig. 3.6A) demonstrated that TBLE suppressed fibril formation. Without $\mathrm{H}_{2} \mathrm{O}_{2}$ or with $5 \times \mathrm{H}_{2} \mathrm{O}_{2}$, fibril bundles were still observed after $8 \mathrm{~h}$ of incubation with TBLE vesicles (Fig. 3.6A), but to a lesser extent compared to when vesicles were absent (Fig. 3.2A). With increasing $\mathrm{H}_{2} \mathrm{O}_{2}$ concentration, the fibril load in the AFM images appeared to decrease. Interestingly, oxidized lipid suppressed fibril formation to a similar extent. With the $1000 \times \mathrm{H}_{2} \mathrm{O}_{2}$ treatment, apparent fibrils developed a halo-like feature around their periphery that was not observed under other conditions (Fig. 3.2A, and Fig. 3.7). This suggests that the combination of lipid species present in TBLE and the high concentration of peroxide alters the peripheral structure of fibrils. This could potentially be due to incorporation of lipids onto the exterior of fibrils or unstructuring of the flanking sequences that encapsulate the polyQ fibril core.

The fibril load across the incubation conditions were quantified as before by determining the number of fibrils per $\mu \mathrm{m}^{2}$, fibril contour length, and the surface area covered by fibrils (Fig. 3.6B-D). As was observed in the absence of lipids, the actual number of discrete fibril aggregates increased with $\mathrm{H}_{2} \mathrm{O}_{2}$ dose, but fibrils formed with increasing $\mathrm{H}_{2} \mathrm{O}_{2}$ concentration were significantly shorter in length. Exposure of unmodified htt to pre-oxidized lipid result in an intermediate increase in fibril population compared to control, but to significantly smaller extent than incubations where the 
peroxide was present. Pre-oxidized lipid also reduced fibril length relative to incubations of htt in the absence of $\mathrm{H}_{2} \mathrm{O}_{2}$ or $5 \times \mathrm{H}_{2} \mathrm{O}_{2}$. Based on the area coverage of fibrils, there was a loose trend of decreased fibril load with increasing peroxide concentration in the presence of vesicles; however, this trend breaks down with $1000 \times \mathrm{H}_{2} \mathrm{O}_{2}$ treatments as these fibrils developed previously mentioned halo around their periphery, which increased the area associated with these aggregates (Fig. 3.6D). Compared with other conditions at similar time point, only htt-exon1(46Q) treated with $1000 \mathrm{x} \mathrm{H}_{2} \mathrm{O}_{2}$ in the presence of vesicles formed these unique features (Fig. 3.7). Pre-oxidized lipids had a similar fibril load to control.
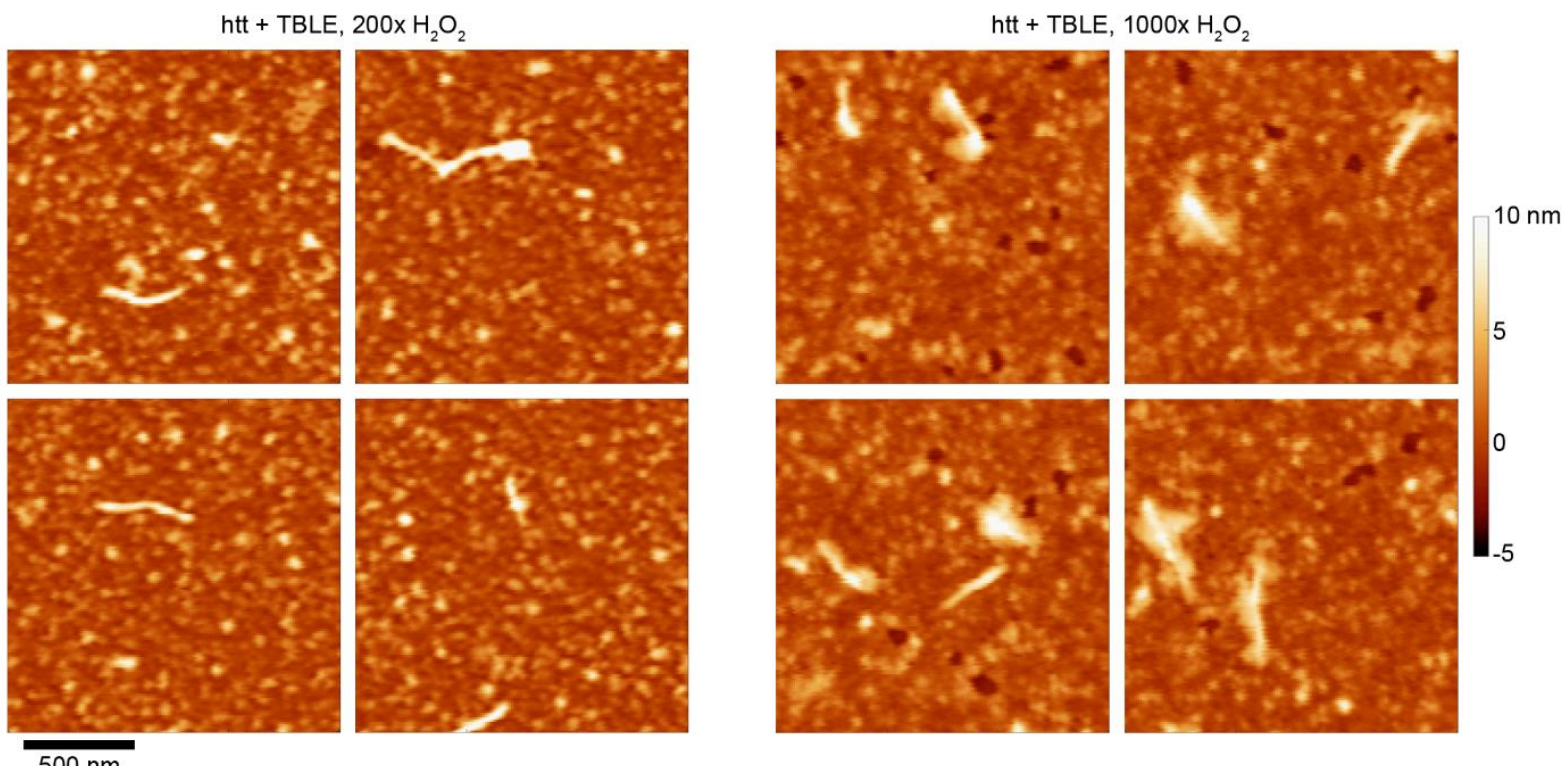

Figure 3.7: Peripheral features formed around htt-exon1(46Q) at extremely high oxidant concentration. Incubations of $20 \mu \mathrm{M}$ htt-exon1(46Q) with $20 \mathrm{mM} \mathrm{H}_{2} \mathrm{O}_{2}$ (1000x) revealed some halo features surrounding fibrils after $8 \mathrm{~h}$. Images from htt-exon 1(46Q) incubations with $4 \mathrm{mM} \mathrm{H}_{2} \mathrm{O}_{2}(200 x)$ after $8 \mathrm{~h}$ are shown for comparison.

Next, we measured the height profiles of the fibrils formed. Compared to similar incubations without lipid, incubations at low oxidant concentrations in the presence of 
TBLE often formed fibrils strands that overlap after $8 \mathrm{~h}$. Single fibrils strands formed in the presence of TBLE vesicles with up to $50 \mathrm{x} \mathrm{H}_{2} \mathrm{O}_{2}$ were similar in thickness to those formed in the absence of lipids, and regions of fibril bundling were as tall as $20-25 \mathrm{~nm}$. However, fibril accumulation/bundling was absent in incubations with higher oxidant concentrations. Single fibril strands formed in the presence of higher $\mathrm{H}_{2} \mathrm{O}_{2}$ concentration (greater than 200x) were less thick compared to fibrils formed in the absence or lower doses of $\mathrm{H}_{2} \mathrm{O}_{2}$, as profiles indicated that these fibrils were typically $3-5 \mathrm{~nm}$ in height, suggesting that the morphology of fibrils formed are altered under high oxidation conditions; however, this is difficult to assess due to the significant suppression of fibril formation under these high oxidative conditions. Supporting the notion that fibril structure is altered with high levels of $\mathrm{H}_{2} \mathrm{O}_{2}$ is the afore mentioned peripheral halo observed around fibrils formed in the presence of TBLE and $1000 \times \mathrm{H}_{2} \mathrm{O}_{2}$.

3.3.4 Oxidative environments influence oligomerization in the presence of lipid vesicles. As oxidation of htt-exon1(46Q) promoted oligomerization in the absence of lipids, the impact of $\mathrm{H}_{2} \mathrm{O}_{2}$ on oligomer formation in the presence TBLE vesicles was investigated. Oligomers in AFM images of incubations of htt in the presence of TBLE vesicles and various treatments of $\mathrm{H}_{2} \mathrm{O}_{2}$ were analyzed using the previously described automated Matlab scripts and criteria. Higher resolution in images of htt-exon1(46Q) oligomers formed in the presence of TBLE vesicles for different incubations in the presence of TBLE vesicles are provided in Fig. 3.8. Under low oxidation conditions (no oxidation, and $5 \mathrm{x}$ ), oligomer population were less than $40 / \mu \mathrm{m}^{2}$ across all time points (Fig. 3.6F). This represents an increase in oligomers associated with the presence of lipids compared to the same conditions without lipids (Fig. 3.2F). At $50 \times \mathrm{H}_{2} \mathrm{O}_{2}$ concentration, 
the oligomer popular was increased significantly to $\sim 60 / \mu \mathrm{m}^{2}$ at all time points. This was also larger than was observed for $50 \mathrm{x}_{2} \mathrm{O}_{2}$ without lipids. While $200 x$ and $1000 \times \mathrm{H}_{2} \mathrm{O}_{2}$ treatments increased the oligomer population in the presence of TBLE vesicles relative to incubations of unmodified htt in the presence of lipids, the number of oligomers at this level of peroxide was smaller compared to incubations of $50 \mathrm{x} \mathrm{H}_{2} \mathrm{O}_{2}$. In addition, the oligomer population formed in the presence of $200 x$ and $1000 x$ was smaller in the presence of TBLE vesicles (Fig. 3.6F) compared to the absence of TBLE vesicles (Fig. 3.2F). Similar to the absence of lipids, treatment with $50 \times \mathrm{H}_{2} \mathrm{O}_{2}$ or larger promoted a broader distribution of oligomer size, i.e. a subset of slightly larger oligomers. However, broadening of the oligomer morphology distribution was not as pronounced in the presence of TBLE vesicles (Fig. 3.6G).

3.3.5 Oxidation alters htt aggregation directly on the TBLE bilayer surface. While both ThT assays and ex situ AFM data revealed that oxidation influences htt aggregation in the presence of TBLE vesicles, these assays only measure aggregation throughout the bulk of the solution. That is, they do not distinguish between aggregation occurring at the membrane interface and in solution. Therefore, in situ AFM experiments were performed to monitor aggregation directly on a supported TBLE bilayer to understand how oxidation impacts aggregation on the membrane surface. Supported lipid bilayers were formed via vesicle fusion. Three different conditions were investigated. Smooth bilayers (as determined by AFM) were exposed to unmodified htt-exon1(46Q) proteins or httexon1(46Q) with $1000 \times \mathrm{H}_{2} \mathrm{O}_{2}$. Control experiments were performed to confirm that exposure to $\mathrm{H}_{2} \mathrm{O}_{2}$ alone did not alter bilayer morphology or integrity (Fig. 3.9). In addition, supported TBLE bilayers that had been pre-oxidized were formed and exposed to 
unmodified htt-exon1(46Q). To cleave the GST moiety, Factor Xa was incubated with the GST-exon1 fusion protein on ice for $90 \mathrm{~min}$ before the protein was exposed to any supported bilayer. The final concentration of htt-exon1(46Q) in the AFM fluid cell was 10 $\mu \mathrm{M}$.

Across all three conditions, distinct morphological changes were observed on the supported lipid bilayers. In the absence of any $\mathrm{H}_{2} \mathrm{O}_{2}$, small oligomers of htt-exon1(46Q) quickly appeared on the bilayer surface (within the first 30 min after exposure, Fig. 3.10A). With time, the bilayer surface around these oligomers displayed a rougher morphology (as can be seen from height profiles, Fig. 3.10B), consistent with disruption of the bilayer packing order. These regions became rougher (due to continued accumulation of additional htt) and more prominent with time (occupying $\sim 25-35 \%$ of the surface area within 150 min of exposure, Fig. 3.10A). In the presence of $1000 \mathrm{x} \mathrm{H}_{2} \mathrm{O}_{2}$, exposure to httexon1(46Q) invoked a distinct morphological change in the bilayer compared with control, especially up to $\sim 80$ min of exposure (Fig. 3.10B). While some small oligomers appeared on the surface, these were associated with raised, plateau-like regions of the bilayer that only appeared under this condition. These regions did not appear in bilayers only exposed to $\mathrm{H}_{2} \mathrm{O}_{2}$ (or as will be seen in pre-oxidized bilayers), indicating that the combination of exposure to peroxide and htt were required to invoke the formation of these domains. Within $~ 80-100$ min after exposure to htt, these plateau-like regions collapsed into a highly granular, disrupted morphology that displayed clear holes within the bilayer. With time, these holes were filled in as more htt accumulated into these regions. Similar to the control experiments, $\sim 25-35 \%$ of the bilayer surface displayed alter morphology due to exposure to htt in the presence of $\mathrm{H}_{2} \mathrm{O}_{2}$, indicating that despite the differences in morphology that 
the total interaction with the bilayer was similar in scale. This is consistent with the PDA assay presented in Fig. 3.5. When pre-oxidized TBLE bilayers were exposed to unmodified htt-exon1(46Q), the plateau-like regions were not observed. Rather, regions with a granular morphology that were associated with htt oligomers developed on the bilayer within 20-50 min (Fig. 3.10A-B). With time, these regions grew in size and accumulated additional material.

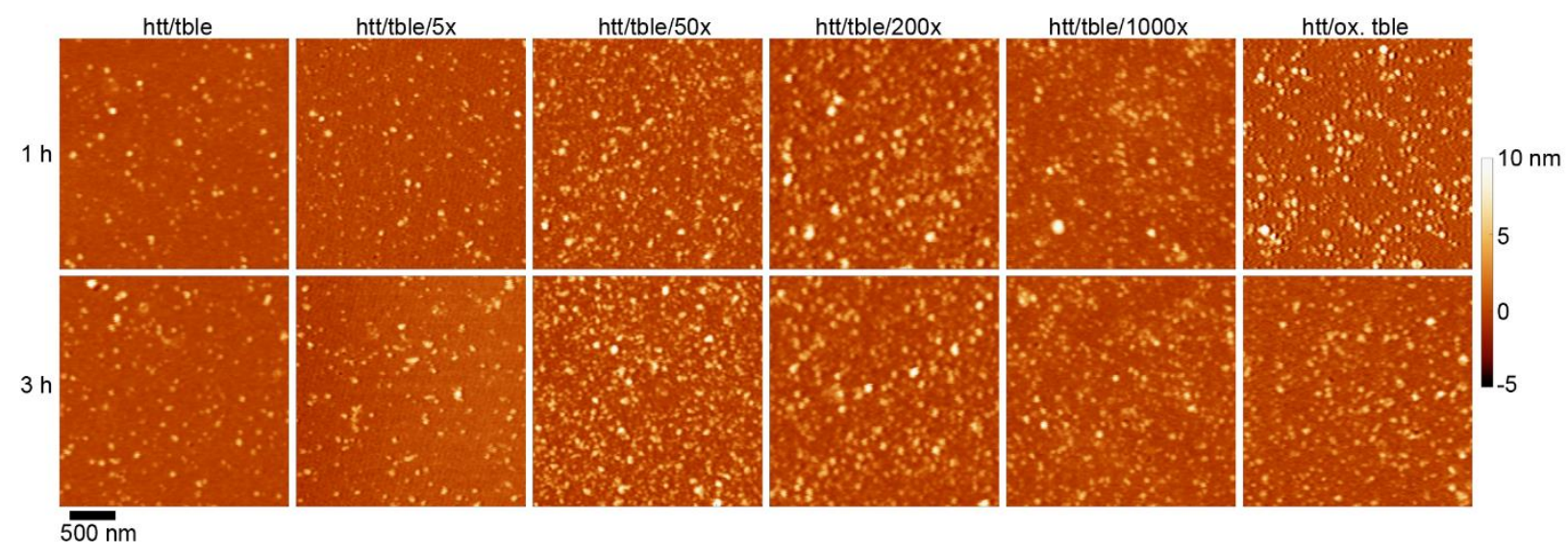

Figure 3.8: Oxidation of htt-exon1(46Q) promoted oligomerization in the presence of TBLE. Zoomed in images taken at 1 and $3 \mathrm{~h}$ showing increased htt-exon1(46Q) oligomerization with increase in oxidation in the presence of TBLE. $20 \mu \mathrm{M} \mathrm{htt-exon1(46Q)}$ + TBLE vesicles were incubated without and with $0.1,1,4$ and $20 \mathrm{mM} \mathrm{H}_{2} \mathrm{O}_{2} .20 \mu \mathrm{M} \mathrm{htt-}$ exon1(46Q) was also incubated with pre-oxidized TBLE. Final TBLE conc. was 300 $\mathrm{mg} / \mathrm{ml}$. 

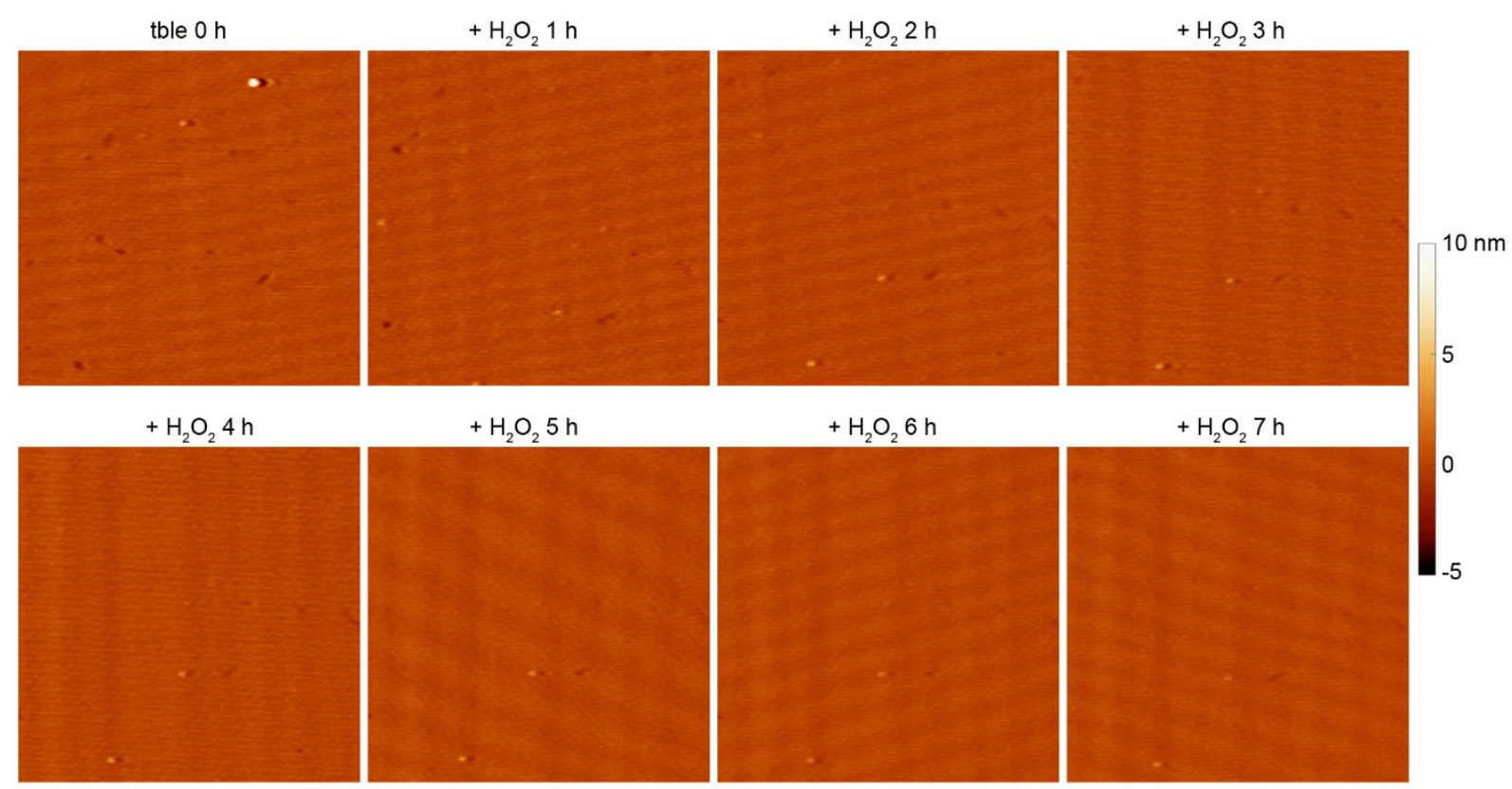

$1 \mu \mathrm{m}$

Figure 3.9: TBLE bilayers stability in the presence of $\mathrm{H}_{2} \mathrm{O}_{2}$. TBLE bilayers were exposed to $20 \mathrm{mM} \mathrm{H}_{2} \mathrm{O}_{2}$ and imaged continuously for $7 \mathrm{~h}$.

To further illustrate the morphological differences of the perturbed bilayer regions associated with exposure to htt-exon1(46Q) under all three conditions, correlation plots of the mean height of disrupted regions vs the mean length across the disrupted regions were constructed (Fig. 3.11). These features were measured for each individual disrupted patch across multiple experiments and grouped by approximate time after exposure to htt. As this analysis was performed on consecutive images obtained from single bilayers, these data represent the evolution of these regions as a function of time. The mean height was determined by averaging the height of all image pixels associated with an identified region of altered morphology. The mean length was defined as the average between the longest and shortest dimension across these features. Based on this analysis, there were clear morphological differences associated with each condition (Fig. 3.11). In the absence 

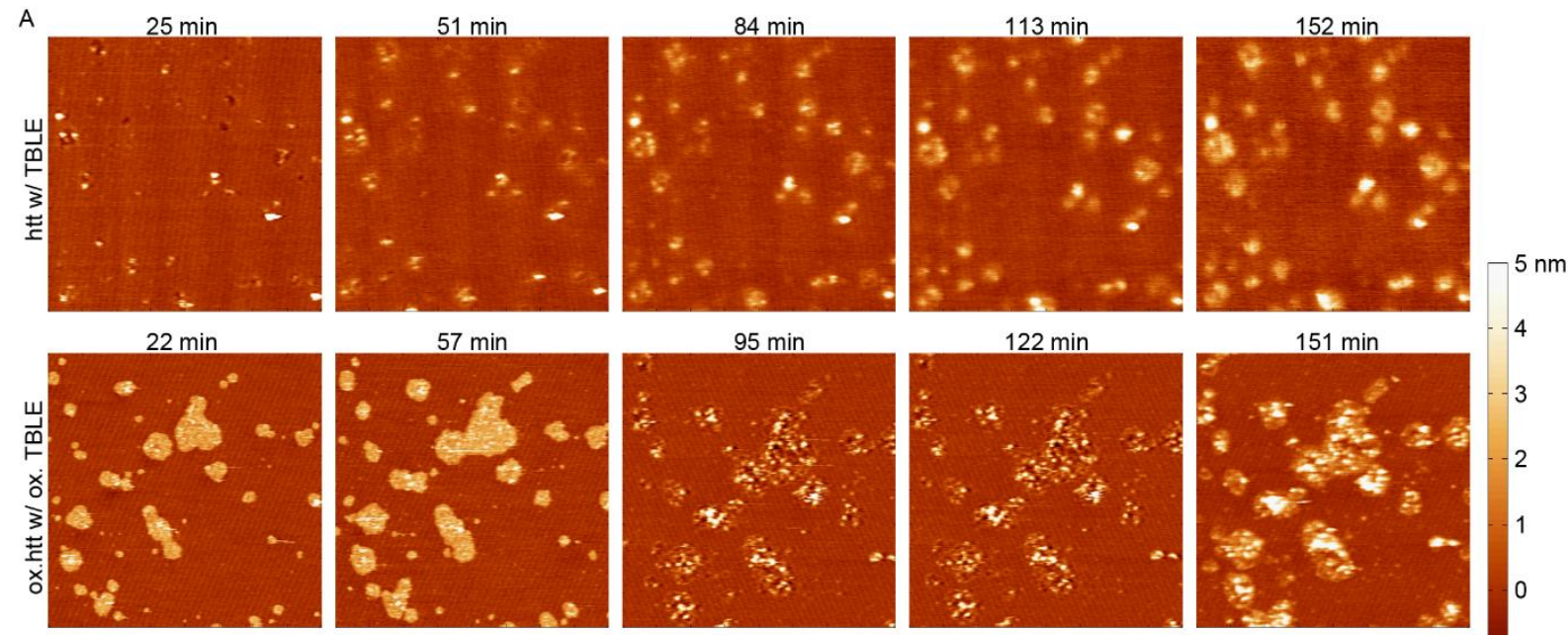

$151 \mathrm{~min}$
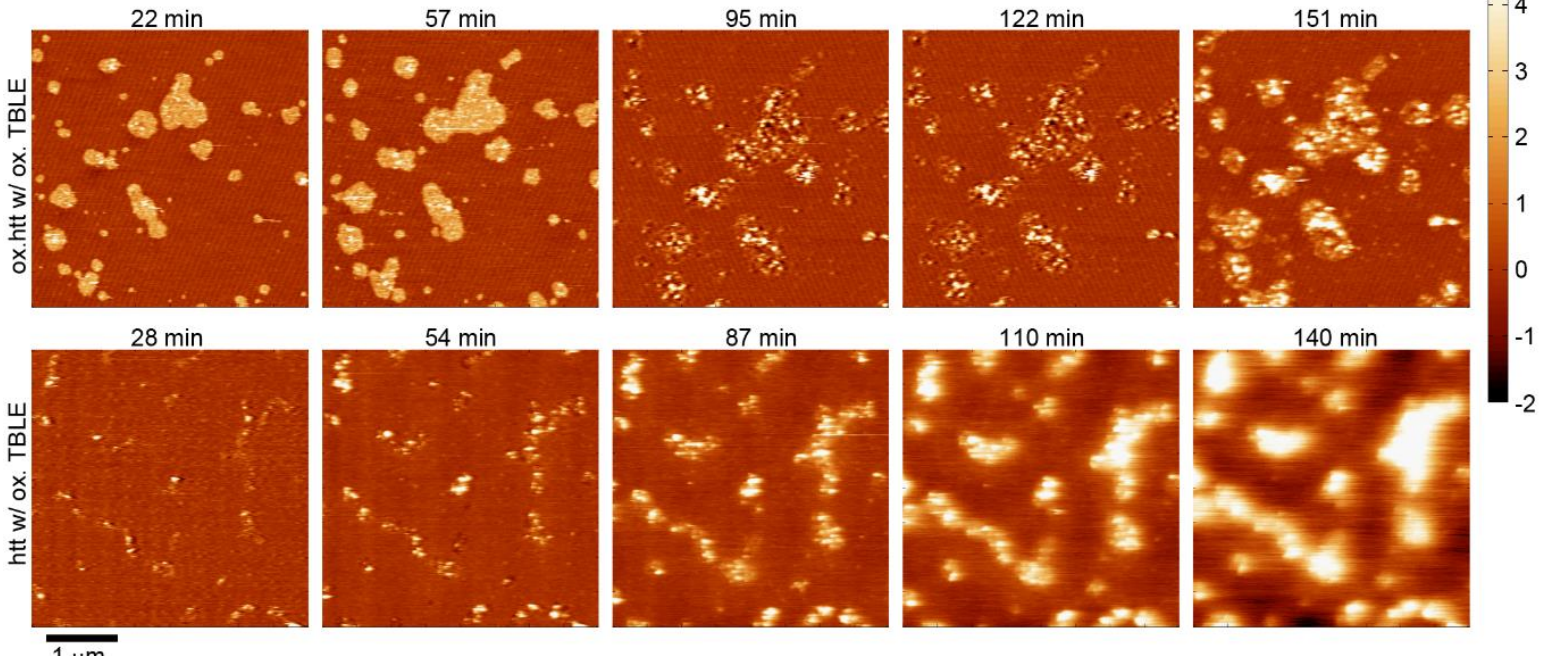

B
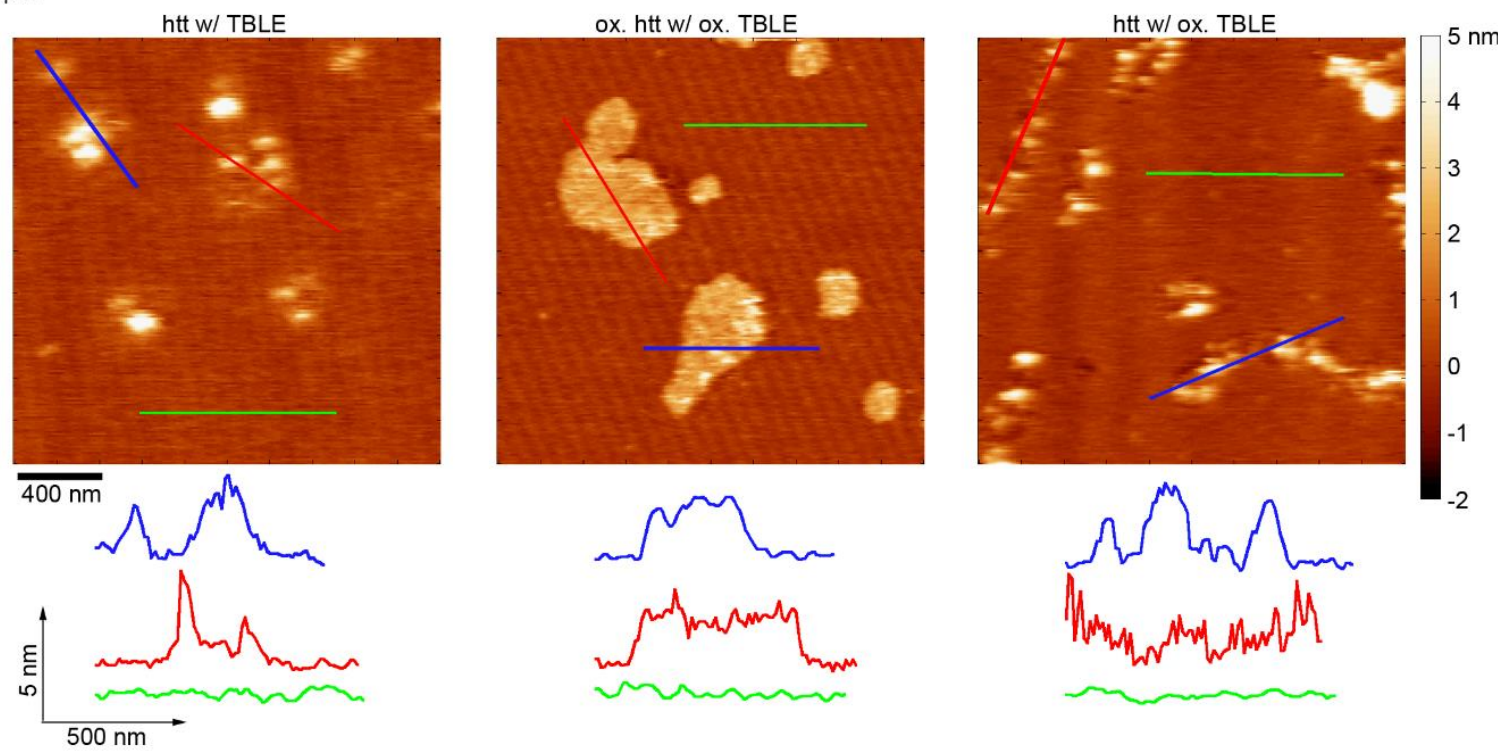

Figure 3.10: Oxidation alters htt aggregation directly on the TBLE bilayer surface. (A) Time-resolved in situ AFM images of supported bilayers of unoxidized and oxidized TBLE exposed to $10 \mu \mathrm{M}$ of htt-exon1(46Q). Both htt-exon1(46Q) and TBLE were also oxidized. (B) Height profiles of the conditions described in A. Blue and red lines represent the profiles of different features observed. Green line represents the profile of the undisrupted region of the bilayers. 

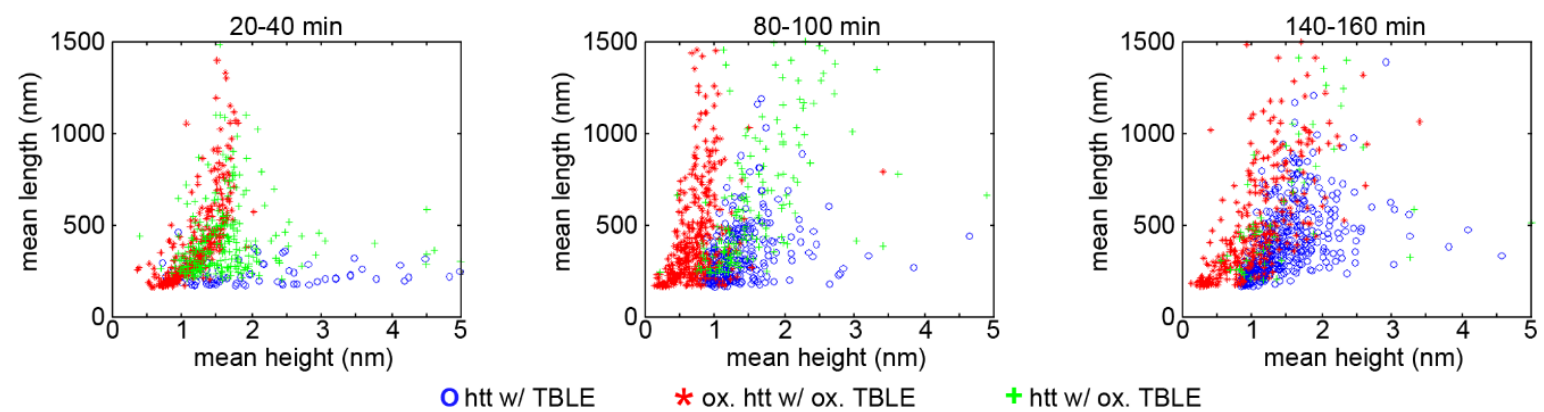

Figure 3.11: Correlation plots showing mean length and mean height of htt aggregates on the surface of TBLE bilayers. Distribution of mean length (average of longest length and shortest length) and mean height of aggregates of (i) htt on unoxidized TBLE (blue), (ii) oxidized htt and oxidized TBLE (red), and (iii) htt on ox TBLE (green) were show at different times.

of $\mathrm{H}_{2} \mathrm{O}_{2}$, regions associated with altered morphology in the first 20-40 min of exposure had a spread of mean height ranging from 1-5 nm but with a tighter distribution of mean length below $\sim 300 \mathrm{~nm}$. This is predominately due to the abundance of discrete oligomers that were not yet associated with large regions of perturbed bilayer morphology. In contrast, at the 20-40 min time frame with both $1000 \times \mathrm{H}_{2} \mathrm{O}_{2}$ present and with pre-oxidized lipid bilayers, the mean height distributions were clustered around $0.5-2 \mathrm{~nm}$, and the distribution of mean length was much broader, extending beyond $1000 \mathrm{~nm}$ in several cases. This is due to larger patches of disruption being observed. After $80-100 \mathrm{~min}$ of htt exposure, the unoxidized and the pre-oxidized lipid conditions displayed similar correlations between mean height and length. This is due to roughened bilayer morphology developing around discrete oligomers in the unoxidized condition and the continued development of grainy patches forming on the pre-oxidized lipid. With the $1000 \mathrm{X} \mathrm{H}_{2} \mathrm{O}_{2}$ condition, there was a clear difference in the correlation plot at 80-100 min as the plateau-like domains collapsing created a clear shift in the mean height (predominately $<1 \mathrm{~nm}$ ). As these disrupted morphological patches on the supported 
bilayer are all susceptible to accumulation of additional protein, the correlation plots for all three conditions have significant overlap after 140-160 min of exposure. Together, these results suggest that the aggregation of htt-exon1(46Q) is specifically altered at the lipid interface by oxidation of both the protein and the lipid.

\subsection{Discussion}

Oxidative stress presents in a number of neurodegenerative diseases associated with amyloid formation, ${ }^{67}$ and oxidation of amyloid-forming proteins alter aggregation and aggregate stability. ${ }^{54,68,69}$ While this study focused on htt-exon1, oxidation of a cysteine residue in an $\mathrm{N}$-terminal fragment of htt longer than exon1 promotes oligomerization. ${ }^{70} \mathrm{In}$ a shorter synthetic htt peptide containing Nt17 residues and various lengths of polyQ stretches, oxidation of M8 to a sulfoxide prevents aggregation. ${ }^{19,51}$ This observation is consistent with our findings that exposure to $\mathrm{H}_{2} \mathrm{O}_{2}$ decreases fibril load in a dose dependent manner while promoting increased number of oligomers. With large doses of $\mathrm{H}_{2} \mathrm{O}_{2}$, the actual number of discrete fibrils observed was larger, but these were significantly shorter, suggesting that fibril elongation was being impacted by the presence of peroxide. Similar to observation under other conditions that reduce htt fibril formation, ${ }^{43,66}$ the oligomers appeared larger as fibril suppression was enhanced by $\mathrm{H}_{2} \mathrm{O}_{2}$, suggesting that some conditions blocking fibril elongation can lead to a slight swelling of oligomer size. A variety of effects on aggregation have been associated with htt oxidation in cellular environments, adding another level of complexity. Cells expressing htt with an expanded polyQ stretch were more sensitive to $\mathrm{H}_{2} \mathrm{O}_{2}$ treatment compared with cells expressing non-pathogenic length $\mathrm{htt}^{71}$ In the pathogenic cell line, $\mathrm{H}_{2} \mathrm{O}_{2}$ treatment increased inclusion formation, suggesting that htt oxidation enhanced aggregation in a 
cellular environment; however, this may be related to impaired proteasome activity associated with oxidative stress. Oxidation of M8 in preformed htt aggregates leads to enhanced inter-aggregate interactions, modifying aggregate morphology. ${ }^{72}$ Interestingly, aggregated forms of htt were more readily oxidized than soluble htt. Oxidation of M8 dissociates htt from the ER membrane, creating soluble htt that is susceptible to phosphorylation, increasing nuclear retention and inclusion formation. ${ }^{44}$ Collectively, these observations suggest oxidation of Nt17 alter htt's interaction with other cellular factors that influence aggregation. With htt being highly associated with membranes in vivo and the Nt17's ability to bind membranes, lipids represent one of these potential cellular factors.

The combination of TBLE and $\mathrm{H}_{2} \mathrm{O}_{2}$ further modified htt aggregation. Peroxide continued to suppress fibril formation in a dose dependent manner in the presence of TBLE, and the $1000 \times \mathrm{H}_{2} \mathrm{O}_{2}$ treatment caused a peripheral halo, potentially comprised of lipid components, to develop around fibrils after $8 \mathrm{~h}$ of incubation. The presence of TBLE vesicles reduced the enhancement of htt oligomers associated with exposure to $\mathrm{H}_{2} \mathrm{O}_{2}$ in their absence. That is, the number of oligomers did not increase as significantly with a much less pronounced swelling of oligomer size as a function of $\mathrm{H}_{2} \mathrm{O}_{2}$ dose. The ability of TBLE to stabilize discrete sizes of htt oligomers has been observed before with an $\mathrm{Nt}^{17}$ Q35 peptide ${ }^{34}$ and with acetylated htt-exon1 $1{ }^{43}$ In particular, acetylation increased oligomer size compared to unmodified htt-exon1 in the absence of lipids, but oligomers were similar in size on TBLE bilayers regardless of acetylation state. ${ }^{43}$ The direct observation, via in situ AFM, that the presence of $\mathrm{H}_{2} \mathrm{O}_{2}$ alters the initial htt-induced morphological changes of TBLE bilayers demonstrates the complexity of htt aggregation in different 
environments. Lipid peroxidation was a contributing factor influencing htt aggregation regardless of the oxidation state of htt itself. When pre-oxidized TBLE was exposed to unmodified htt-exon1, morphological changes on the bilayer also varied from unoxidized controls. Specifically, the accumulation of htt within these altered regions of the bilayer was enhanced. This enhancement may be an indication of increased membrane defects associated with lipid peroxidation that leads to enhanced membrane fluidity. ${ }^{73}$ For example, when lipids containing POPC and cholesterol were rapidly oxidized, domains of disordered oxidized lipids form. ${ }^{74}$ Oxidized lipid tails can migrate toward hydrophilic head groups, ${ }^{75}$ creating additional membrane defects that may be susceptible to htt accumulation. Determining all the factors influencing aggregation in vivo is daunting, but the interfaces between subcellular membranes and the cytosol represent a unique environment that can promote unique aggregation pathways. ${ }^{37,76}$

Amphipathic $\alpha$-helical motifs commonly function to sense membrane curvature,,$^{77,78}$ and Nt17 displays a preference for curved membranes in vitro. ${ }^{79}$ The binding of amphipathic $\alpha$-helical motifs to lipid membranes are often regulated by PTMs. In this regard, the large variety of PTMs that occur in Nt17 may play a role in both htt's normal functions and toxic gain of function associated with polyQ expansion. For example, phosphorylation of $\mathrm{Nt}^{17}$ regulates nuclear export of $\mathrm{htt} 30,80$ and promotes clearance of htt via the proteasome and lysosome. ${ }^{45}$ PTMs in Nt17 indeed manipulate its helical content. ${ }^{81}$ Oxidation of M8 in Nt17 functions as a ROS sensor, altering htt's affinity for the ER membrane, and triggering localization to the nucleus. ${ }^{44}$ Despite this effect of promoting detachment from the ER, oxidation of $M 8$ promoted $\alpha$-helical content. ${ }^{44}$ Molecular dynamic simulations suggest that when $\mathrm{Nt17}$ is pre-folded into an $\alpha$-helix that 
it binds membranes more readily. ${ }^{82}$ However, $M 8$ is positioned facing toward the hydrophobic core when bound to POPC bilayers, ${ }^{21}$ and the advanced hydrophilicity associated with oxidation would make this positioning less favorable. Indeed, oxidation reduces the affinity of Nt17 for LPC/LPG micelles by $\sim 3-4$ fold; however, the $\alpha$-helix of micelle-bound NT17 extends by an additional 2 residues. ${ }^{51}$ Based on these reports and our observation of altered aggregation of $\mathrm{H}_{2} \mathrm{O}_{2}$ treated htt, we expected to observe a decreased binding of htt with TBLE upon oxidation. However, the oxidation did not significantly reduce the extent of htt/lipid interaction. The root cause of this discrepancy may lie in the unique lipid systems used, as lipid composition heavily influences htt aggregation as will be discussed in the next paragraph. Despite a similar magnitude of htt/lipid interaction, the initial htt aggregation process occurring directly on the TBLE bilayer surface was clearly altered based on in situ AFM assays.

Selectivity of Nt17 for different subcellular membranes throughout cells may derive from a combination of varying lipid composition and PTMs of htt. Once bound, these unique lipid environments exert influence on the aggregation process through their physicochemical properties, as observed in a variety of amyloid/membrane interactions. ${ }^{76}$ TBLE and other brain extracts slow fibril formation. ${ }^{50,66,83}$ Adding exogenous cholesterol, sphingomyelin, or gangliosides to TBLE modifies htt aggregation directly on supported bilayers, resulting in the appearance of unique morphological and mechanical changes. POPC/POPS lipid systems promote htt fibril formation and a unique aggregation mechanism in comparison to aggregation occurring in bulk solution. ${ }^{28,37}$ Zwitterionic lipids POPC and POPE mildly altered the rate of htt aggregation, with POPC mildly enhancing aggregation and POPE mildly slowing it. ${ }^{41}$ However, anionic lipids (POPG and POPS) 
strongly accelerate fibril formation, and POPG promoted a unique fibril morphology. ${ }^{41}$ The variation in how specific lipids interact with htt via Nt17 represents a plausible explanation as to why oxidation reduced htt affinity for LPC/LPG micelles, ${ }^{51}$ while we observed no significant reduction in the ability of oxidized htt to bind TBLE. The ability of a single PTM in Nt17 to influence htt/lipid binding in a lipid dependent manner is observed in studies using phosphomimetic mutations in htt-exon1 ${ }^{50}$ Phosphomimetic mutations at T3 and S13 significantly reduced the binding of htt-exon1 to TBLE vesicles, but these mutations had no significant impact on the ability of htt to bind POPC or POPG vesicles.

In summary, $\mathrm{H}_{2} \mathrm{O}_{2}$ reduced total fibril formation of htt in a dose dependent manner, and reduced fibril elongation appeared to be a prominent feature of this phenomenon. This promoted a larger population of oligomers with slightly altered morphology. In the presence of TBLE vesicles based on bulk solution assays, $\mathrm{H}_{2} \mathrm{O}_{2}$ retained its ability to reduce htt fibril load in a dose dependent manner; however, the impact on oligomer formation was reduced. This occurred despite no change in the bulk affinity of htt for TBLE vesicles. Nevertheless, oxidation environments altered htt-induced morphological changes and aggregation directly at the bilayer surface. 


\subsection{References}

(1) MacDonald, M. E., Ambrose, C. M., Duyao, M. P., Myers, R. H., Lin, C., Srinidhi, L., Barnes, G., Taylor, S. A., James, M., Groot, N., MacFarlane, H., Jenkins, B., Anderson, M. A., Wexler, N. S., Gusella, J. F., Bates, G. P., Baxendale, S., Hummerich, H., Kirby, S., North, M., Youngman, S., Mott, R., Zehetner, G., Sedlacek, Z., Poustka, A., Frischauf, A. M., Lehrach, H., Buckler, A. J., Church, D., Doucette-Stamm, L., O'Donovan, M. C., Riba-Ramirez, L., Shah, M., Stanton, V. P., Strobel, S. A., Draths, K. M., Wales, J. L., Dervan, P., Housman, D. E., Altherr, M., Shiang, R., Thompson, L., Fielder, T., Wasmuth, J. J., Tagle, D., Valdes, J., Elmer, L., Allard, M., Castilla, L., Swaroop, M., Blanchard, K., Collins, F. S., Snell, R., Holloway, T., Gillespie, K., Datson, N., Shaw, D., and Harper, P. S. (1993) A novel gene containing a trinucleotide repeat that is expanded and unstable on Huntington's disease chromosomes. Cell 72, 971-983.

(2) Adegbuyiro, A., Sedighi, F., Pilkington, A. W., Groover, S., and Legleiter, J. (2017) Proteins Containing Expanded Polyglutamine Tracts and Neurodegenerative Disease. Biochemistry 56, 1199-1217.

(3) Wacker, J. L., Zareie, M. H., Fong, H., Sarikaya, M., and Muchowski, P. J. (2004) $\mathrm{Hsp} 70$ and Hsp40 attenuate formation of spherical and annular polyglutamine oligomers by partitioning monomer. Nature Structural and Molecular Biology 11, 1215-1222.

(4) Legleiter, J., Mitchell, E., Lotz, G. P., Sapp, E., Ng, C., DiFiglia, M., Thompson, L. M., and Muchowski, P. J. (2010) Mutant huntingtin fragments form oligomers in a polyglutamine length-dependent manner in Vitro and in Vivo. Journal of Biological Chemistry 285, 14777-14790.

(5) Poirier, M. A., Li, H., Macosko, J., Cai, S., Amzel, M., and Ross, C. A. (2002) Huntingtin spheroids and protofibrils as precursors in polyglutamine fibrilization. Journal of Biological Chemistry 277, 41032-41037.

(6) Chen, S., Berthelier, V., Hamilton, J. B., O'Nuallain, B., and Wetzel, R. (2002) Amyloidlike features of polyglutamine aggregates and their assembly kinetics. Biochemistry 41, 7391-7399.

(7) Kim, Y. E., Hosp, F., Mann, M., Hayer-hartl, M., and Hartl, F. U. (2016) Soluble Oligomers of PolyQ-Expanded Huntingtin Target a Multiplicity of Key Cellular Factors Article Soluble Oligomers of PolyQ-Expanded Huntingtin Target a Multiplicity of Key Cellular Factors. Molecular Cell 63, 951-964.

(8) Nucifora, L. G., Burke, K. A., Feng, X., Arbez, N., Zhu, S., Miller, J., Yang, G., Ratovitski, T., Delannoy, M., Muchowski, P. J., Finkbeiner, S., Legleiter, J., Ross, C. A., and Poiriera, M. A. (2012) Identification of novel potentially toxic oligomers formed in vitro from mammalian-derived expanded huntingtin exon-1 protein. Journal of Biological Chemistry 287, 16017-16028. 
(9) Lajoie, P., and Snapp, E. L. (2010) Formation and toxicity of soluble polyglutamine oligomers in living cells. PLOS ONE 5, e15245.

(10) Drombosky, K. W., Rode, S., Kodali, R., Jacob, T. C., Palladino, M. J., and Wetzel, R. (2018) Mutational analysis implicates the amyloid fibril as the toxic entity in Huntington's disease. Neurobiology of Disease 120, 126-138.

(11) Pieri, L., Madiona, K., Bousset, L., and Melki, R. (2012) Fibrillar a -Synuclein and Huntingtin Exon 1 Assemblies Are Toxic to the Cells. Biophysj 102, 2894-2905.

(12) Saha, I., Mishra, A., Hartl, F. U., and Baumeister, W. (2017) In Situ Architecture and Cellular Interactions of PolyQ Inclusions Article In Situ Architecture and Cellular Interactions of PolyQ Inclusions. Cell 171, 179-187.

(13) Ueda, M., Li, S., Itoh, M., Wang, M., Hayakawa, M., Islam, S., Nakagawa, K., Chen, H., and Nakagawa, T. (2016) Biochemical and Biophysical Research Communications Expanded polyglutamine embedded in the endoplasmic reticulum causes membrane distortion and coincides with Bax insertion. Biochemical and Biophysical Research Communications 474, 259-263.

(14) Choo, Y. S., Johnson, G. V. W., MacDonald, M., Detloff, P. J., and Lesort, M. (2004) Mutant huntingtin directly increases susceptibility of mitochondria to the calcium-induced permeability transition and cytochrome c release. Human Molecular Genetics 13, 14071420.

(15) Panov, A. v., Gutekunst, C.-A., Leavitt, B. R., Hayden, M. R., Burke, J. R., Strittmatter, W. J., and Greenamyre, J. T. (2002) Early mitochondrial calcium defects in Huntington's disease are a direct effect of polyglutamines. Nature Neuroscience 5, 731736.

(16) Liu, K. Y., Shyu, Y. C., Barbaro, B. A., Lin, Y. T., Chern, Y., Thompson, L. M., Shen, C. K. J., and Marsh, J. L. (2015) Disruption of the nuclear membrane by perinuclear inclusions of mutant huntingtin causes cell-cycle re-entry and striatal cell death in mouse and cell models of Huntington's disease. Human Molecular Genetics 24, 1602-1616.

(17) Jayaraman, M., Kodali, R., Sahoo, B., Thakur, A. K., Mayasundari, A., Mishra, R., Peterson, C. B., and Wetzel, R. (2012) Slow amyloid nucleation via a-helix-rich oligomeric intermediates in short polyglutamine-containing huntingtin fragments. Journal of Molecular Biology 415, 881-899.

(18) Bhattacharyya, A., Thakur, A. K., Chellgren, V. M., Thiagarajan, G., Williams, A. D., Chellgren, B. W., Creamer, T. P., and Wetzel, R. (2006) Oligoproline effects on polyglutamine conformation and aggregation. Journal of Molecular Biology 355, 524-535.

(19) Thakur, A. K., Jayaraman, M., Mishra, R., Thakur, M., Chellgren, V. M., Byeon, I.-J. L., Anjum, D. H., Kodali, R., Creamer, T. P., Conway, J. F., Gronenborn, A. M., and Wetzel, R. (2009) Polyglutamine disruption of the huntingtin exon $1 \mathrm{~N}$ terminus triggers a complex aggregation mechanism. Nature structural \& molecular biology 16, 380-9. 
(20) Mishra, R., Jayaraman, M., Roland, B. P., Landrum, E., Fullam, T., Kodali, R., Thakur, A. K., Arduini, I., and Wetzel, R. (2012) Inhibiting the nucleation of amyloid structure in a huntingtin fragment by targeting a-helix-rich oligomeric intermediates. Journal of Molecular Biology 415, 900-917.

(21) Michalek, M., Salnikov, E. S., and Bechinger, B. (2013) Structure and Topology of the Huntingtin $1-17$ Membrane Anchor by a Combined Solution and Solid-State NMR Approach. Biophysj 105, 699-710.

(22) Arndt, J. R., Kondalaji, S. G., Maurer, M. M., Parker, A., Legleiter, J., and Valentine, S. J. (2015) Huntingtin N-Terminal Monomeric and Multimeric Structures Destabilized by Covalent Modification of Heteroatomic Residues. Biochemistry 54, 4285-4296.

(23) Kotler, S. A., Tugarinov, V., Schmidt, T., Ceccon, A., Libich, D. S., Ghirlando, R., Schwieters, C. D., and Marius Clore, G. (2019) Probing initial transient oligomerization events facilitating Huntingtin fibril nucleation at atomic resolution by relaxation-based NMR. Proceedings of the National Academy of Sciences of the United States of America 116, 3562-3571.

(24) Wetzel, R. (2012) Physical chemistry of polyglutamine: Intriguing tales of a monotonous sequence. Journal of Molecular Biology 421, 466-490.

(25) DiFiglia, M., Sapp, E., Chase, K., Schwarz, C., Meloni, A., Young, C., Martin, E., Vonsattel, J. P., Carraway, R., Reeves, S. A., Boyce, F. M., and Aronin, N. (1995) Huntingtin is a cytoplasmic protein associated with vesicles in human and rat brain neurons. Neuron 14, 1075-1081.

(26) Velier, J., Kim, M., Schwarz, C., Kim, T. W., Sapp, E., Chase, K., Aronin, N., and DiFiglia, M. (1998) Wild-type and mutant huntingtins function in vesicle trafficking in the secretory and endocytic pathways. Experimental Neurology 152, 34-40.

(27) Kegel, K. B., Kim, M., Sapp, E., McIntyre, C., Castano, J. G., Aronin, N., and DiFiglia, M. (2000) Huntingtin expression stimulates endosomal-lysosomal activity, endosome tubulation, and autophagy. Journal of Neuroscience 20, 7268-7278.

(28) Tao, M., Pandey, N. K., Barnes, R., Han, S., and Langen, R. (2019) Structure of Membrane-Bound Huntingtin Exon 1 Reveals Membrane Interaction and Aggregation Mechanisms. Structure 27, 1570-1580.e4.

(29) Rockabrand, E., Slepko, N., Pantalone, A., Nukala, V. N., Kazantsev, A., Marsh, J. L., Sullivan, P. G., Steffan, J. S., Sensi, S. L., and Thompson, L. M. (2007) The first 17 amino acids of Huntingtin modulate its sub-cellular localization, aggregation and effects on calcium homeostasis. Human Molecular Genetics 16, 61-77.

(30) Atwal, R. S., Xia, J., Pinchev, D., Taylor, J., Epand, R. M., and Truant, R. (2007) Huntingtin has a membrane association signal that can modulate huntingtin aggregation, nuclear entry and toxicity. Human Molecular Genetics 16, 2600-2615. 
(31) Chang, D. T. W., Rintoul, G. L., Pandipati, S., and Reynolds, I. J. (2006) Mutant huntingtin aggregates impair mitochondrial movement and trafficking in cortical neurons. Neurobiology of Disease 22, 388-400.

(32) Shirendeb, U. P., Calkins, M. J., Manczak, M., Anekonda, V., Dufour, B., McBride, J. L., Mao, P., and Reddy, P. H. (2012) Mutant Huntingtin's interaction with mitochondrial protein Drp1 impairs mitochondrial biogenesis and causes defective axonal transport and synaptic degeneration in Huntington's disease. Human Molecular Genetics 21, 406-420.

(33) Ruan, Q., Lesort, M., MacDonald, M. E., and Johnson, G. V. W. (2004) Striatal cells from mutant huntingtin knock-in mice are selectively vulnerable to mitochondrial complex II inhibitor-induced cell death through a non-apoptotic pathway. Human Molecular Genetics 13, 669-681.

(34) Burke, K. A., Kauffman, K. J., Umbaugh, C. S., Frey, S. L., and Legleiter, J. (2013) The interaction of polyglutamine peptides with lipid membranes is regulated by flanking sequences associated with huntingtin. Journal of Biological Chemistry 288, 1499315005.

(35) Suopanki, J., Götz, C., Lutsch, G., Schiller, J., Harjes, P., Herrmann, A., and Wanker, E. E. (2006) Interaction of huntingtin fragments with brain membranes - Clues to early dysfunction in Huntington's disease. Journal of Neurochemistry 96, 870-884.

(36) Eckmann, J., Clemens, L. E., Eckert, S. H., Hagl, S., Yu-Taeger, L., Bordet, T., Pruss, R. M., Muller, W. E., Leuner, K., Nguyen, H. P., and Eckert, G. P. (2014) Mitochondrial Membrane Fluidity is Consistently Increased in Different Models of Huntington Disease: Restorative Effects of Olesoxime. Molecular Neurobiology 50, 107-118.

(37) Pandey, N. K., Isas, J. M., Rawat, A., Lee, R. v., Langen, J., Pandey, P., and Langen, R. (2017) The 17-residue-long $N$ terminus in huntingtin controls step-wise aggregation in solution and on membranes via different mechanisms. Journal of Biological Chemistry 293, 2597-2605.

(38) Burke, K. A., Kauffman, K. J., Umbaugh, C. S., Frey, S. L., and Legleiter, J. (2013) The interaction of polyglutamine peptides with lipid membranes is regulated by flanking sequences associated with huntingtin. Journal of Biological Chemistry 288, 1499315005.

(39) Chaibva, M., Gao, X., Jain, P., Campbell, W. A., Frey, S. L., and Legleiter, J. (2017) Sphingomyelin and GM1 Influence Huntingtin Binding to, Disruption of, and Aggregation on Lipid Membranes. ACS Omega 3, 273-285.

(40) Gao, X., Campbell, W. A., Chaibva, M., Jain, P., Leslie, A. E., Frey, S. L., and Legleiter, J. (2016) Cholesterol Modifies Huntingtin Binding to, Disruption of, and Aggregation on Lipid Membranes. Biochemistry 55, 92-102. 
(41) Beasley, M., Groover, S., Valentine, S. J., and Legleiter, J. (2021) Lipid headgroups alter huntingtin aggregation on membranes. Biochimica et Biophysica Acta Biomembranes 1863, 183497.

(42) Sedighi, F., Adegbuyiro, A., and Legleiter, J. (2020) SUMOylation Prevents Huntingtin Fibrillization and Localization onto Lipid Membranes. ACS Chemical Neuroscience 11, 328-343.

(43) Chaibva, M., Jawahery, S., Pilkington, A. W., Arndt, J. R., Sarver, O., Valentine, S., Matysiak, S., and Legleiter, J. (2016) Acetylation within the First 17 Residues of Huntingtin Exon 1 Alters Aggregation and Lipid Binding. Biophysical Journal 111, 349362.

(44) DiGiovanni, L. F., Mocle, A. J., Xia, J., and Truant, R. (2016) Huntingtin N17 domain is a reactive oxygen species sensor regulating huntingtin phosphorylation and localization. Human Molecular Genetics 25, 3937-3945.

(45) Thompson, L. M., Aiken, C. T., Kaltenbach, L. S., Agrawal, N., Illes, K., Khoshnan, A., Martinez-Vincente, M., Arrasate, M., O'Rourke, J. G., Khashwji, H., Lukacsovich, T., Zhu, Y. Z., Lau, A. L., Massey, A., Hayden, M. R., Zeitlin, S. O., Finkbeiner, S., Green, K. N., LaFerla, F. M., Bates, G., Huang, L., Patterson, P. H., Lo, D. C., Cuervo, A. M., Marsh, J. L., and Steffan, J. S. (2009) IKK phosphorylates Huntingtin and targets it for degradation by the proteasome and lysosome. Journal of Cell Biology 187, 1083-1099.

(46) Aiken, C. T., Steffan, J. S., Guerrero, C. M., Khashwji, H., Lukacsovich, T., Simmons, D., Purcell, J. M., Menhaji, K., Zhu, Y. Z., Green, K., LaFerla, F., Huang, L., Thompson, L. M., and Marsh, J. L. (2009) Phosphorylation of threonine 3: Implications for huntingtin aggregation and neurotoxicity. Journal of Biological Chemistry 284, 29427-29436.

(47) Cong, X., Held, J. M., DeGiacomo, F., Bonner, A., Chen, J. M., Schilling, B., Czerwieniec, G. A., Gibson, B. W., and Ellerby, L. M. (2011) Mass spectrometric identification of novel lysine acetylation sites in Huntingtin. Molecular and Cellular Proteomics 10, 1-13.

(48) Steffan, J. S., Agrawal, N., Pallos, J., Rockabrand, E., Trotman, L. C., Slepko, N., Illes, K., Lukacsovich, T., Zhu, Y. Z., Cattaneo, E., Pandolfi, P. P., Thompson, L. M., and Marsh, J. L. (2004) SUMO Modification of Huntingtin and Huntington's Disease Pathology. Science 304, 100-104.

(49) Kalchman, M. A., Graham, R. K., Xia, G., Brook Koide, H., Graeme Hodgson, J., Graham, K. C., Paul Goldberg, Y., Dan Gietz, R., Pickart, C. M., and Hayden, M. R. (1996) Huntingtin is ubiquitinated and interacts with a specific ubiquitin- conjugating enzyme. Journal of Biological Chemistry 271, 19385-19394.

(50) Groover, S. E., Beasley, M., Ramamurthy, V., and Legleiter, J. (2020) Phosphomimetic Mutations Impact Huntingtin Aggregation in the Presence of a Variety of Lipid Systems. Biochemistry 59, 4681-4693. 
(51) Ceccon, A., Schmidt, T., Tugarinov, V., Kotler, S. A., Schwieters, C. D., and Clore, G. M. (2018) Interaction of Huntingtin Exon-1 Peptides with Lipid-Based Micellar Nanoparticles Probed by Solution NMR and Q-Band Pulsed EPR. Journal of the American Chemical Society 140, 6199-6202.

(52) Ceccon, A., Tugarinov, V., and Clore, G. M. (2019) TiO 2 Nanoparticles Catalyze Oxidation of Huntingtin Exon 1-Derived Peptides Impeding Aggregation: A Quantitative NMR Study of Binding and Kinetics. Journal of the American Chemical Society 141, 9497.

(53) Chen, S. (2001) Solubilization and disaggregation of polyglutamine peptides. Protein Science 10, 887-891.

(54) Pilkington, A. W., Valentine, S. J., Legleiter, J., Donohoe, G. C., Akhmedov, N. G., and Ferrebee, T. (2019) Hydrogen peroxide modifies $A \beta$-membrane interactions with implications for A 440 aggregation. Biochemistry 58, 2893-2905.

(55) Beasley, M., Stonebraker, A. R., and Legleiter, J. (2020) Normalizing polydiacetylene colorimetric assays of vesicle binding across lipid systems. Analytical Biochemistry 609, 113864.

(56) Zheng, F., Wu, Z., and Chen, Y. (2012) A quantitative method for the measurement of membrane affinity by polydiacetylene-based colorimetric assay. Analytical Biochemistry 420, 171-176.

(57) Sokolovski, M., Sheynis, T., Kolusheva, S., and Jelinek, R. (2008) Membrane interactions and lipid binding of casein oligomers and early aggregates. Biochimica et Biophysica Acta - Biomembranes 1778, 2341-2349.

(58) Horcas, I., Fernández, R., Gómez-Rodríguez, J. M., Colchero, J., Gómez-Herrero, J., and Baro, A. M. (2007) WSXM: A software for scanning probe microscopy and a tool for nanotechnology. Review of Scientific Instruments 78, 1-8.

(59) Burke, K. A., Godbey, J., and Legleiter, J. (2011) Assessing mutant huntingtin fragment and polyglutamine aggregation by atomic force microscopy. Methods 53, 275284.

(60) LeVine, H. (1995) Thioflavine T interaction with amyloid $\beta$-sheet structures. Amyloid 2, 1-6.

(61) Sivanandam, V. N., Jayaraman, M., Hoop, C. L., Kodali, R., Wetzel, R., and van der Wel, P. C. A. (2011) The aggregation-enhancing huntingtin $\mathrm{N}$-terminus is helical in amyloid fibrils. Journal of the American Chemical Society 133, 4558-4566.

(62) Lin, H., Boatz, J. C., Krabbendam, I. E., Kodali, R., Hou, Z., Wetzel, R., Dolga, A. M., Poirier, M. A., and Wel, P. C. A. van der. (2017) Fibril polymorphism affects immobilized non-amyloid flanking domains of huntingtin exon1 rather than its polyglutamine core. Nature Communications 8, 1-12. 
(63) Kegel-Gleason, K. B. (2013) Huntingtin interactions with membrane phospholipids: Strategic targets for therapeutic intervention? Journal of Huntington's Disease 2, 239250.

(64) Kegel, K. B., Sapp, E., Yoder, J., Cuiffo, B., Sobin, L., Kim, Y. J., Qin, Z. H., Hayden, M. R., Aronin, N., Scott, D. L., Isenberg, G., Goldmann, W. H., and DiFiglia, M. (2005) Huntingtin associates with acidic phospholipids at the plasma membrane. Journal of Biological Chemistry 280, 36464-36473.

(65) Kegel, K. B., Sapp, E., Alexander, J., Valencia, A., Reeves, P., Li, X., Masso, N., Sobin, L., Aronin, N., and DiFiglia, M. (2009) Polyglutamine expansion in huntingtin alters its interaction with phospholipids. Journal of Neurochemistry 110, 1585-1597.

(66) Beasley, M., Stonebraker, A. R., Hasan, I., Kapp, K. L., Liang, B. J., Agarwal, G., Groover, S., Sedighi, F., and Legleiter, J. (2019) Lipid Membranes Influence the Ability of Small Molecules to Inhibit Huntingtin Fibrillization. Biochemistry 58, 4361-4373.

(67) Barnham, K. J., Masters, C. L., and Bush, A. I. (2004) Neurodegenerative diseases and oxidatives stress. Nature Reviews Drug Discovery 3, 205-214.

(68) Göbl, C., Morris, V. K., van Dam, L., Visscher, M., Polderman, P. E., Hartlmüller, C., de Ruiter, H., Hora, M., Liesinger, L., Birner-Gruenberger, R., Vos, H. R., Reif, B., Madl, T., and Dansen, T. B. (2020) Cysteine oxidation triggers amyloid fibril formation of the tumor suppressor p16INK4A. Redox Biology 28, 101316.

(69) Scarlata, S., and Golebiewska, U. (2014) Linking alpha-synuclein properties with oxidation: a hypothesis on a mechanism underling cellular aggregation. Journal of Bioenergetics and Biomembranes 46, 93-98.

(70) Fox, J. H., Connor, T., Stiles, M., Kama, J., Lu, Z., Dorsey, K., Liebermann, G., Sapp, E., Cherny, R. A., Banks, M., Volitakis, I., DiFiglia, M., Berezovska, O., Bush, A. I., and Hersch, S. M. (2011) Cysteine oxidation within N-terminal mutant huntingtin promotes oligomerization and delays clearance of soluble protein. Journal of Biological Chemistry 286, 18320-18330.

(71) Goswami, A., Dikshit, P., Mishra, A., Mulherkar, S., Nukina, N., and Jana, N. R. (2006) Oxidative stress promotes mutant huntingtin aggregation and mutant huntingtindependent cell death by mimicking proteasomal malfunction. Biochemical and Biophysical Research Communications 342, 184-190.

(72) Mitomi, Y., Nomura, T., Kurosawa, M., Nukina, N., and Furukawa, Y. (2012) Postaggregation oxidation of mutant huntingtin controls the interactions between aggregates. Journal of Biological Chemistry 287, 34764-34775.

(73) Yusupov, M., Wende, K., Kupsch, S., Neyts, E. C., Reuter, S., and Bogaerts, A. (2017) Effect of head group and lipid tail oxidation in the cell membrane revealed through integrated simulations and experiments. Scientific Reports 7, 1-14. 
(74) Haluska, C. K., Baptista, M. S., Fernandes, A. U., Schroder, A. P., Marques, C. M., and Itri, R. (2012) Photo-activated phase separation in giant vesicles made from different lipid mixtures. Biochimica et Biophysica Acta - Biomembranes 1818, 666-672.

(75) Wong-Ekkabut, J., Xu, Z., Triampo, W., Tang, I. M., Tieleman, D. P., and Monticelli, L. (2007) Effect of lipid peroxidation on the properties of lipid bilayers: A molecular dynamics study. Biophysical Journal 93, 4225-4236.

(76) Burke, K. A., Yates, E. A., and Legleiter, J. (2013) Biophysical insights into how surfaces, including lipid membranes, modulate protein aggregation related to neurodegeneration. Frontiers in Neurology 4, 1-17.

(77) Drin, G., and Antonny, B. (2010) Amphipathic helices and membrane curvature. FEBS Letters 584, 1840-1847.

(78) Drin, G., Casella, J. F., Gautier, R., Boehmer, T., Schwartz, T. U., and Antonny, B. (2007) A general amphipathic $\alpha$-helical motif for sensing membrane curvature. Nature Structural and Molecular Biology 14, 138-146.

(79) Chaibva, M., Burke, K. A., and Legleiter, J. (2014) Curvature enhances binding and aggregation of huntingtin at lipid membranes. Biochemistry 53, 2355-2365.

(80) Maiuri, T., Woloshansky, T., Xia, J., and Truant, R. (2013) The huntingtin N17 domain is a multifunctional CRM1 and ran-dependent nuclear and cilial export signal. Human Molecular Genetics 22, 1383-1394.

(81) Yalinca, H., Gehin, C. J. C., Oleinikovas, V., Lashuel, H. A., Gervasio, F. L., and Pastore, A. (2019) The Role of Post-translational Modifications on the Energy Landscape of Huntingtin N-Terminus. Frontiers in Molecular Biosciences 6, 1-7.

(82) Karanji, A. K., Beasley, M., Sharif, D., Ranjbaran, A., Legleiter, J., and Valentine, S. J. (2020) Investigating the interactions of the first 17 amino acid residues of Huntingtin with lipid vesicles using mass spectrometry and molecular dynamics. Journal of mass spectrometry: JMS 55, e4470.

(83) Levy, G. R., Shen, K., Gavrilov, Y., Smith, P. E. S., Levy, Y., Chan, R., Frydman, J., and Frydman, L. (2019) Huntingtin's N-Terminus Rearrangements in the Presence of Membranes: A Joint Spectroscopic and Computational Perspective. ACS Chemical Neuroscience 10, 472-481. 


\section{Future directions for mitigating huntingtin aggregation and toxicity}

\subsection{Introduction}

Huntington's disease is a fatal autosomal dominant neurodegenerative disease caused by an expansion mutation of polyglutamine (polyQ) stretch located near the $\mathrm{N}$ terminus of the huntingtin (htt) protein. Expansion of the polyQ domain correlates with $\mathrm{htt}$ aggregation into oligomers, fibrils, and amorphous aggregates. Ultimately, these aggregate species accumulate to form inclusion bodies in the nucleus and cytosol. ${ }^{1-3}$ Toxicity has been attributed, both individually and collectively, to all these aggregate species. ${ }^{4-9}$ Due to the potential toxic activity of all these unique aggregate species, unraveling the complexity of the heterogeneous htt aggregation process would prove critical in understanding htt-related toxic mechanisms. Importantly, several cellular factors such as the presence of lipid membranes and post translational modification of htt profoundly contribute to the aggregation process. ${ }^{1}$ In this dissertation, the impact of mitochondrial lipids and oxidation on htt aggregation were determined.

In the cellular environment, lipid membranes enclosing organelles provide unique surface/cytosolic interfaces. While htt localizes to numerous membranous surfaces, ${ }^{10,11}$ the interaction of htt with mitochondrial surfaces are thought to play a key role in $\mathrm{HD}$, as mitochondrial dysfunction is a marker in disease progression. Most studies focus on the consequence to mitochondria integrity, dynamics, and function upon exposure to mutant htt; ${ }^{10,12-14}$ however, little is known about how the mitochondrial environment influences htt aggregation. In Chapter 2, we sought to understand how the presence of MEFs and mitochondrial membrane mimics impact htt aggregation. The presence of MEFs isolated from brain or liver reduced htt fibrillization and promoted a unique oligomer morphology. 
Mitochondrial membrane mimic vesicles influenced aggregation in a similar fashion, that is, reduction in htt fibril formation in the presence of OMM and IMM mimics. The IMM was more effective at suppressing fibrillization. A systematic investigation into the difference in the degrees of fibrillization inhibition by OMM and IMM implicated CL, a lipid unique to mitochondria but more abundant in IMM, in playing a key role in reducing fibrillization. Interestingly, direct observation of htt aggregation on the surface of OMM or IMM demonstrated that morphological changes on the membranes were starkly different. While htt formed granular, oligomeric and fibrillar aggregates on OMM, large domains of htt that were void of distinct morphology developed on IMM. Collectively, these observations suggest that the mitochondrial environment strongly influences htt aggregation. These distinct aggregate species may play a role in htt-induced mitochondrial dysfunction.

A major factor in $\mathrm{HD}$ and many other neurodegenerative diseases is aging, which can be associated with consequential oxidative stress. ${ }^{15-18}$ Under stressed conditions, there are more reactive oxidative species formed than consumed. This excess oxidant load promotes oxidative PTM of proteins. ${ }^{19-21}$ In $\mathrm{HD}$, the $\mathrm{Nt} 17$, or the $17 \mathrm{~N}$-terminal residues of huntingtin, contains methionine residues that are prone to oxidation. This same region (Nt17) of htt functions as a membrane binding domain and plays a key role in initiating the aggregation process. Thus, htt oxidation, like other PTMs in Nt17, may have profound consequences for htt membrane localization. ${ }^{22-24}$ However, little is known about the impact of oxidation on htt aggregation. In Chapter 3 , the aim was to determine the impact of oxidative environments, using $\mathrm{H}_{2} \mathrm{O}_{2}$, on htt aggregation and membrane interactions. As verified by Thioflavin T assay and ex situ AFM, increasing oxidant 
concentration progressively reduced the total amount of fibrils formed in the absence of lipids. Fibrils formed under highly oxidative environments were shorter, suggesting that oxidation impedes fibril elongation. However, higher oxidant concentrations increased oligomer formation and stability. The presence of lipids (oxidized and unoxidized) complicated the aggregation of htt in an oxidative environment. There was a reduction in the total load of fibrils formed as $\mathrm{H}_{2} \mathrm{O}_{2}$ concentration was increased and the morphology of the fibrils was altered. While the presence of lipids and $\mathrm{H}_{2} \mathrm{O}_{2}$ also facilitated the formation of short fibrils, fibrillization was generally suppressed and a peripheral halo was observed around the fibrils formed at very high oxidation concentration. Similarly, the oligomers population in these oxidative environments were altered in comparison to htt controls. Oligomerization of htt was promoted $\mathrm{H}_{2} \mathrm{O}_{2}$ by in the presence or absence of TBLE vesicles. In addition, at the membrane interface, the presence of $\mathrm{H}_{2} \mathrm{O}_{2}$ influenced morphological changes upon exposure to htt. In the absence of oxidants, htt deposited as oligomeric aggregates that increased in both size and number with time. In contrast, when both htt and TBLE were subjected to high oxidative environment, the formation of plateau-like patches of htt deposits that were rich in granular and oligomer species were formed. The exposure of htt to oxidized TBLE showed similar htt deposition that recapitulates morphological features in unoxidized systems. That is, there were formation of granular oligomeric aggregates that accumulated more aggressively. Taken together, the work in Chapter 3 suggested that oxidation of htt alter the morphology of fibrils formed, regardless of the presence or absence of lipids. In addition, in the presence of htt, oxidative environment appeared to promote the formation of domains in TBLE bilayers. 
As research into a cure for HD continues, it is important to fully understand the consequence of lipid synthesis, peroxidation and htt interactions in HD, and to discover therapeutic approaches that will reduce htt toxicity. Based on the works presented in Chapters 2 and 3, it becomes imperative to: 1) determine the impact of cardiolipin on htt toxicity in cells; 2) investigate the effect of oxidation of some select lipids that make up mitochondrial membranes, and the consequence on htt aggregation; 3) determine the contributions of other cellular factors such as molecular crowding; and 4) understand how protein/protein interactions impact htt aggregation.

\subsection{Impact of Cardiolipin on htt toxicity}

$\mathrm{CL}$ plays a crucial role in maintaining the integrity of mitochondrial membranes, providing support, and being involved in mitochondria fission. Cardiolipin content may vary per location in response to cellular stress. For example, CL becomes externalized from the inner mitochondrial membrane to the outer membrane during mitophagy. ${ }^{25,26}$ Unsurprisingly, CL decrease or alteration has been implicated in apoptosis ${ }^{27}$ and numerous diseases ${ }^{28-31}$ including neurodegenerative diseases. ${ }^{32,33}$ Specifically, the amount of $\mathrm{CL}$ in brain of transgenic Alzheimer's Disease mice fall significantly compared with non-transgenic mice. ${ }^{32}$ More recently, we have demonstrated that CL suppresses the fibrillization of htt. As htt aggregation is central to HD pathology, and consensus on the roles of the different aggregate species on htt toxicity is not yet in sight, it becomes important to understand the role of this lipid in htt toxicity. If the strong correlation between CL content and htt aggregation observed in vitro with ThT assay and ex situ AFM also holds in vivo, and if this translates into reduced toxicity, CL content regulation may 
become a therapeutic strategy for HD. A strategy to investigate this is to control the synthesis of $C L$ in cell lines expressing wild type and mutant htt.

\subsection{Investigation of the role of oxidized lipids of mitochondria on htt aggregation}

Mitochondria is a source of ROS, producing superoxide radicals which later generate hydrogen peroxide ${ }^{34,35}$ during different stages of respiration. Mitochondria also susceptible to oxidation as evidenced by lipid and protein peroxidation. ${ }^{36-38}$ The most abundant lipid in mammalian mitochondria is neutral $P C,{ }^{39}$ which is susceptible to oxidation. ${ }^{40}$ Also unique to mitochondria is cardiolipin with an anionic net charge and in most instances a high degree of unsaturation in its acyl chains, which are consequently prone to oxidation. The fluidity of membranes containing oxidized lipids increases. ${ }^{41}$ There is also migration of the oxidized regions from the membrane interior to the surface, ${ }^{42}$ promoting unique surface domains and defects. In Chapter 2, we showed that mitochondrial membranes impact huntingtin aggregation. In Chapter 3 , it was demonstrated that oxidation also modifies htt aggregation and its ability to interact directly with lipid membranes. With the production of ROS at mitochondrial surfaces, these two chapters collectively point toward a need to understand how oxidation influences htt aggregation in the presence of mitochondria. For example, will htt aggregation be inhibited if some of the lipids of mitochondria are oxidized? (Fig. 4.1).

In addition, mitochondrial dysfunction is implicated in HD and it is a site of ROS production, it would be beneficial to ascertain how oxidation of mitochondrial lipids impact mitochondrial membrane integrity. This can be achieved by oxidizing separately PC (the most abundant lipid in mitochondria) and CL (the unique mitochondrial lipid which often 


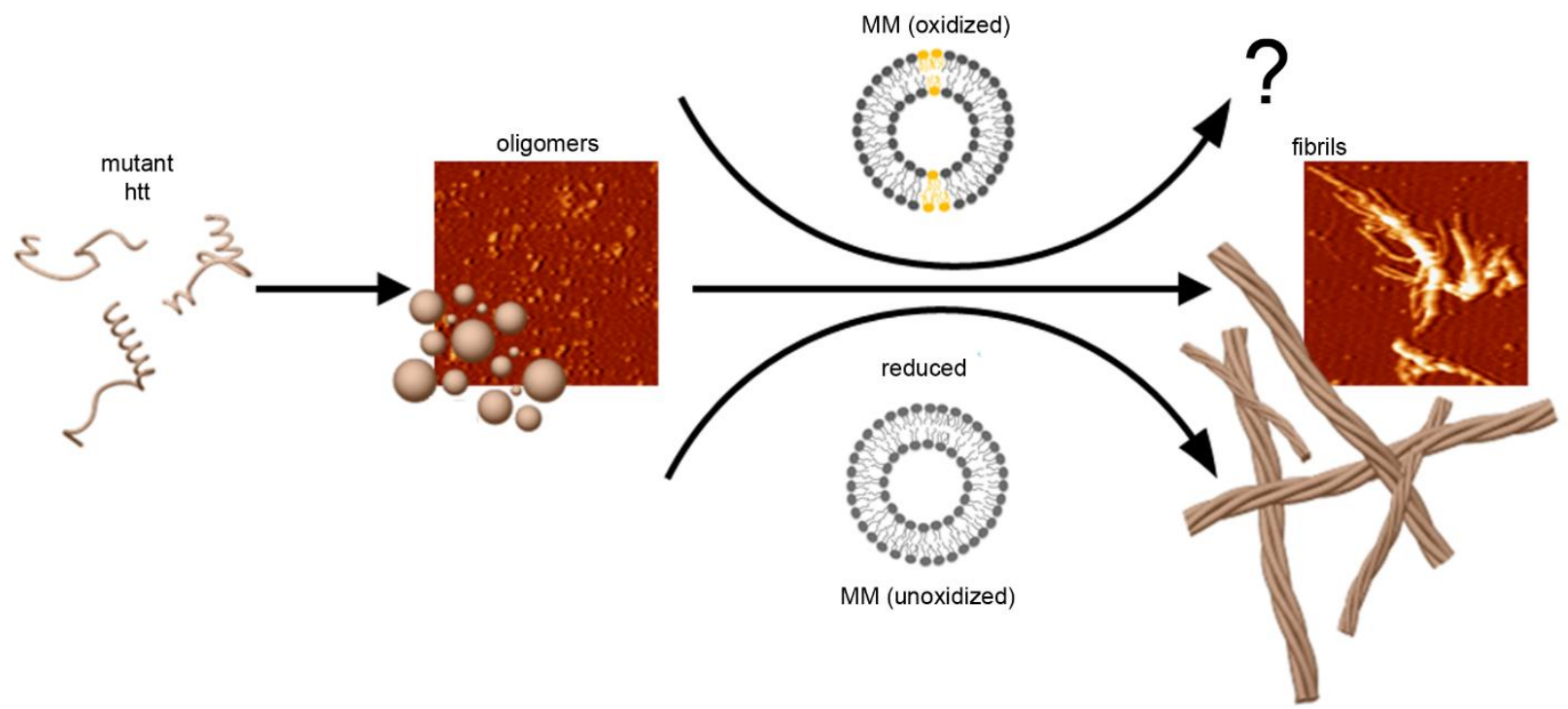

Figure 4.1. Determination of effect of oxidized lipids of mitochondria on htt aggregation. Htt aggregates into oligomers and fibrils. In the presence of mitochondrial membrane mimic (MM), fibrillization of htt was reduced (bottom arrow). The impact of oxidized lipids of mitochondria (top arrow) is unknown.

exist with high degree of unsaturation in acyl tail) prior to vesicles and bilayers formation. Experimentation can be performed as described in Chapter 2, that is, exposure of htt to vesicles made from oxidized and unoxidized lipids of mitochondria. Aliquots of aggregating mixtures can be drawn, deposited on mica, and imaged with AFM for aggregates morphology determination. Also, the consequence of oxidized PC and CL on the integrity of mitochondrial membrane can be investigated by forming bilayers from the vesicles and probing bilayer surface with AFM for mechanical changes. 


\subsection{How does crowding affect huntingtin aggregation?}

In Chapters 2 and 3, we investigated the interaction of htt at membrane interfaces using in situ AFM. The variations in the bilayers systems used include lipid composition and chemical modifications. From the studies presented in the earlier chapters, the deposition of htt to the surface of lipid bilayers varied, further supporting evidence about the differences various membranes impact on protein aggregation. Various surfaces can trigger conformational changes in proteins, hence steer the aggregation towards a unique or different path as compared to aggregation in free solution. ${ }^{43-49}$ Such changes may be as a result of multiple interactions such as electrostatics and Van der Waals. ${ }^{50}$ Aside from the contribution of phase difference and interactions at membrane interfaces, crowding of the protein environment also contributes to aggregation ${ }^{51}$ through a variety of mechanisms, i.e. excluded volumes effects. ${ }^{52}$ Excluded volume effects are the stearic hinderances that exist on a macromolecule in relation to others as a consequence of the coexistence of multiple macromolecules or crowders. In practice, movement is limited as a result of excluded volumes, causing a molecule to experience less randomness or entropy. Alteration in a thermodynamic property, such as entropy, of a protein may in turn change the behavior of such macromolecule, and consequently, alter protein aggregation and amyloid formation. ${ }^{53-55}$ The cytosol is crowded with various macromolecules such as proteins, polysaccharides, DNA, and RNA. ${ }^{56}$ This creates the scenario where the most physiologically relevant membrane interface is adjacent to a crowded aqueous environment.

Huntingtin is mostly cytosolic but is also found in the nucleus. Intracellularly, huntingtin exist with and interact with multiple organelles and proteins. Since several 
factors can alter the conformation and stability of proteins, and consequently their aggregation, it is expected that an understanding of the aggregation of htt under a controlled crowded environment will provide insights into the rate, and mechanism of htt aggregation into different aggregate species. This hypothesis can be investigated using inert macromolecular crowders such as dextran, Ficoll and PEG. We have performed experiments aimed at understanding the aggregation of surfaces and membrane interfaces with a crowded aqueous phase using in situ AFM. These experiments support the notion that the crowded cytosol can influence aggregation at surfaces. For example, when investigating the aggregation of a synthetic htt peptide mimic $\left(\mathrm{Nt}^{17} \mathrm{Q}_{35} \mathrm{P}_{10} \mathrm{KK}\right)$ on mica, aggregation was inhibited in a crowder-dependent fashion (Fig 4.2A). In the absence of crowders, $\mathrm{Nt}^{17} \mathrm{Q}_{35} \mathrm{P}_{10} \mathrm{KK}$ aggregates appeared as early as $9 \mathrm{~min}$, forming oligomers, and fibrils that increased with time. The addition of crowders caused reduced aggregation on mica, leading to decreased coverage area of aggregates (Fig 4.2B). PEG inhibited fibrillization of $\mathrm{Nt}^{17} \mathrm{Q}_{35} \mathrm{P}_{10} \mathrm{KK}$ the most, with only a few small oligomers observed after $3 \mathrm{~h}$. Ficoll and dextran also reduced aggregation of htt to various degrees.

When the mica surface was substituted with a bilayer of TBLE, a crowded aqueous phase influenced, beyond the rate of aggregation, morphological changes associated with exposure to htt (Fig. 4.3). With a non-crowded aqueous phase, htt formed oligomers that increased in number and were associated with patches of increased roughness on the bilayer surface (Fig. 4.3B). With a crowded aqueous phase, a variety of distinct aggregation patterns were observed on the bilayer surface (Fig. 4.3A-C). Crowders' presence promoted roughness of the membranes (Fig. 4.3B). With the addition of dextran htt also formed oligomers that increased significantly in diameter with time (Fig. 4.3A, C). 


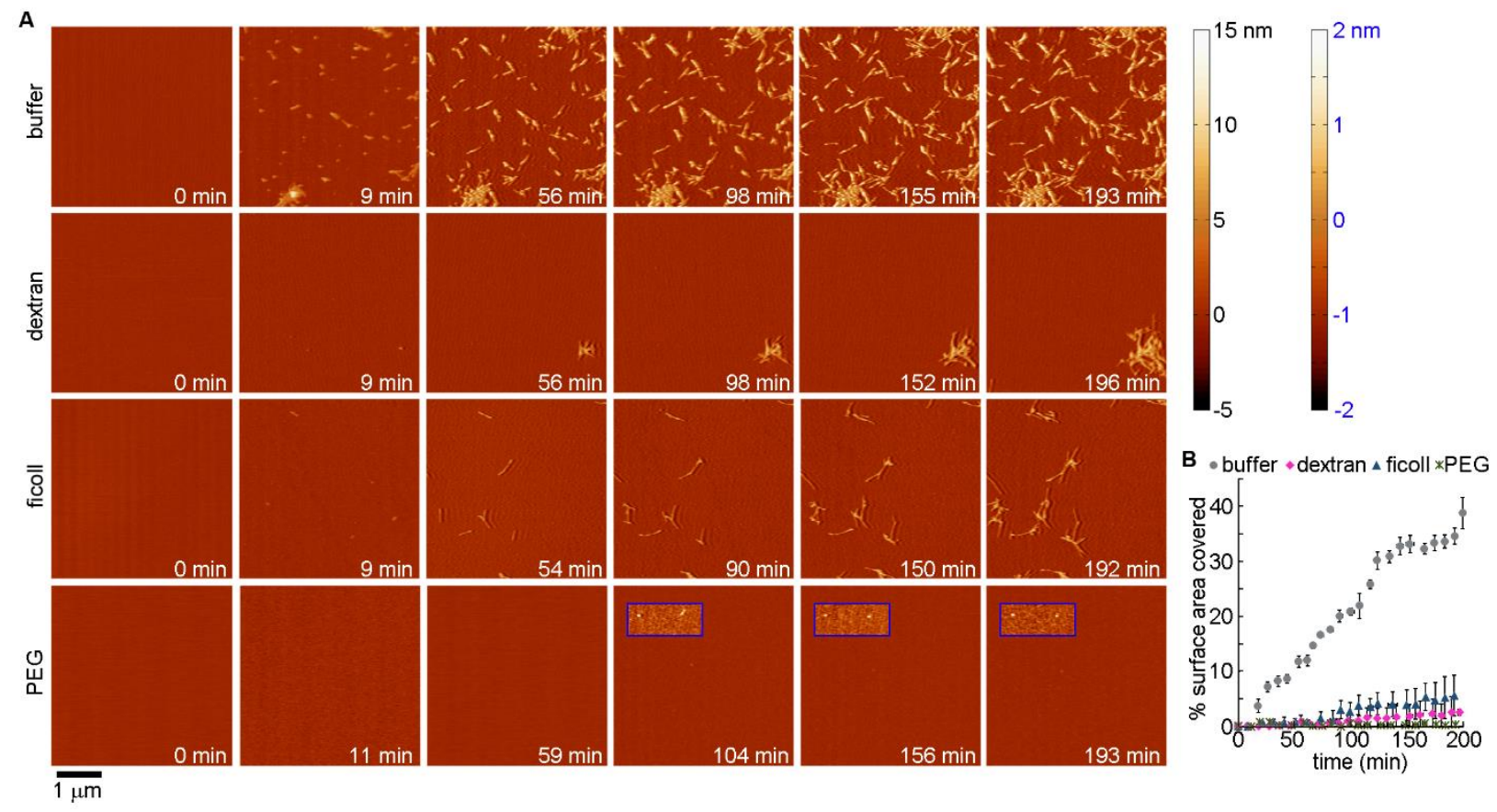

Figure 4.2. Comparison of $\mathrm{Nt}^{17} \mathrm{Q}_{35}-\mathrm{P}_{10}-\mathrm{KK}$ aggregation on mica with different macromolecular crowders in the aqueous phase. (A) Time-sequence, representative in situ AFM images of $\mathrm{Nt}^{17}-Q_{35}-P_{10}-K K(10 \mu M)$ aggregating on mica with buffer, dextran, Ficoll, or PEG in the aqueous phase. The macromolecular crowder concentration was $100 \mathrm{mg} / \mathrm{mL}$. As the height of features varied, the blue boxes represent areas of the image that correspond to the color scale with blue font. (B) Quantification of the \% surface area covered by $\mathrm{Nt}^{17}-\mathrm{Q}_{35}-\mathrm{P}_{10}-\mathrm{KK}$ aggregates as a function of time. Error bars represent standard deviation.

Ficoll promoted the formation of tall globular aggregates that swelled with time, and deep holes eventually developed on the bilayer surface (Fig. 4.3A, C). With PEG, small oligomers initially appeared, and the entire bilayer surfaces began to display enhanced roughness (Fig. 4.3A, B). Large aggregates appeared after $\sim 1 \mathrm{~h}$ and were accompanied by an intense disruption of TBLE bilayer that caused exposure of the underlying mica surface. Accordingly, htt disrupted a larger surface area in the presence of crowders (Fig 4.3 A, D). The highest percent disruption was observed with PEG, which also has the highest degree of roughness. 

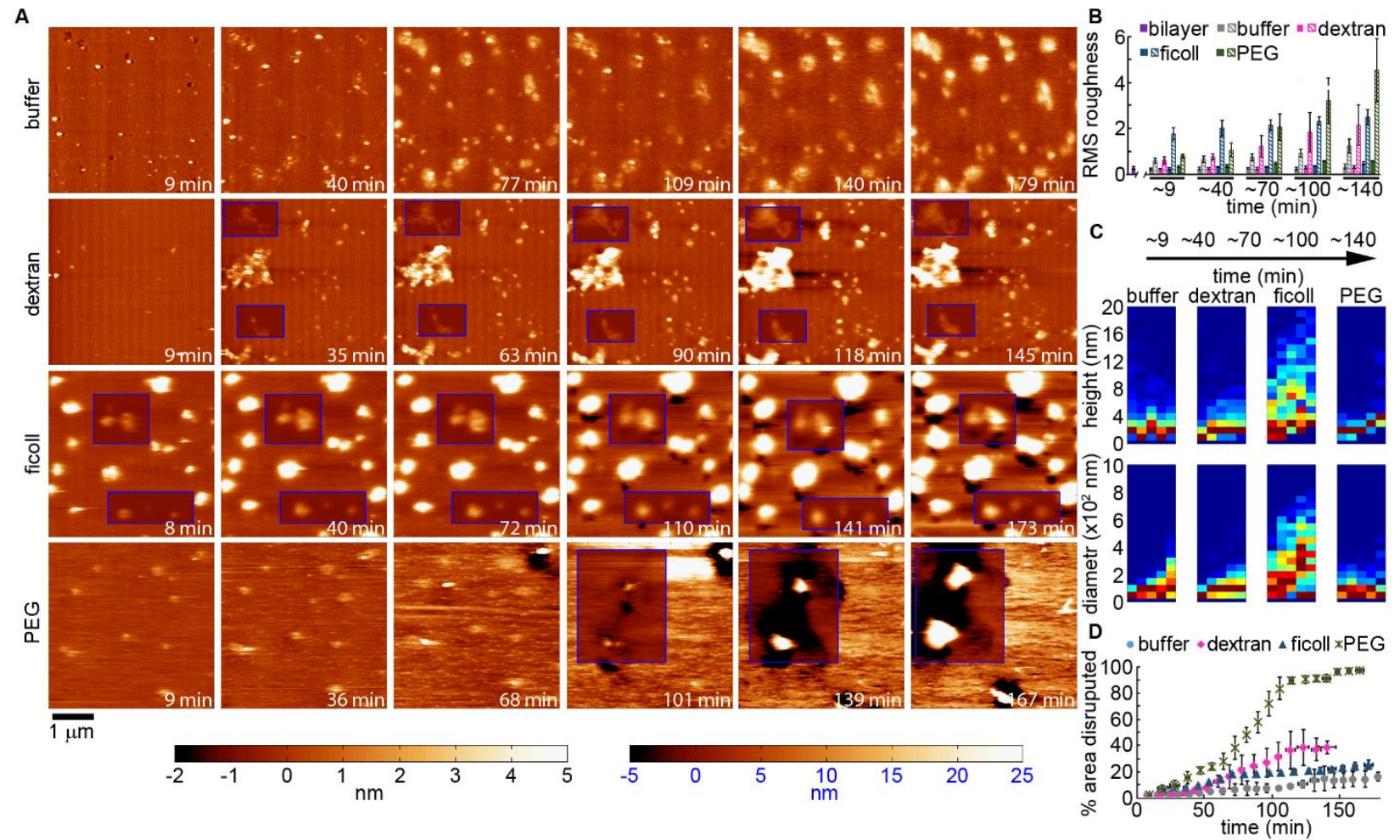

Figure 4.3. Comparison of htt-exon1(46Q) aggregation on supported TBLE bilayers with different macromolecular crowders in the aqueous phase. Time-sequence, representative in situ AFM images of htt-exon1(46Q) (10 $\mu \mathrm{M})$ aggregating on supported TBLE bilayers with buffer, dextran, Ficoll, or PEG in the aqueous phase. The macromolecular crowder concentration was $25 \mathrm{mg} / \mathrm{mL}$. As the height of features varied, the blue boxes represent areas of the image that correspond to the color scale with blue font. (B) Quantification of the RMS roughness of unperturbed (solid bars) and disrupted (striped bars) regions of the bilayer. The RMS roughness of a bilayer prior to exposure to htt-exon1(46Q) is provided by the purple bar. Error bars represent standard deviation. (C) Height and diameter histograms of htt-exon1(46Q) aggregates as a function of time. (D) Quantification of the \% area of the bilayer disrupted by htt-exon1(46Q) as a function of time. Error bars represent standard deviation.

\subsection{Inhibiting amyloid formation through protein-protein interactions.}

Aggregation of proteins are implicated in numerous neurodegenerative diseases.

In crowded cellular environments, these proteins are exposed to numerous influences from various non-specific interactions with macromolecules such as polysaccharides, 
DNA, and even membranous surfaces. In amyloid formation, amyloidogenic proteins misfold, precipitate and deposit in patients through protein-protein interactions. Since proteins are mostly labile, such interactions can upset their stability and consequently alter their aggregation rate. While most amyloids are formed by self-assembly of similar proteins, protein-protein interactions between different proteins may also impact the formation of aggregates. Many amyloid-forming proteins display a variety of specific protein-protein interactions. For example, $\alpha$-synuclein, a protein whose aggregated forms found in Lewy bodies is associated with PD, interacts with multiple proteins.
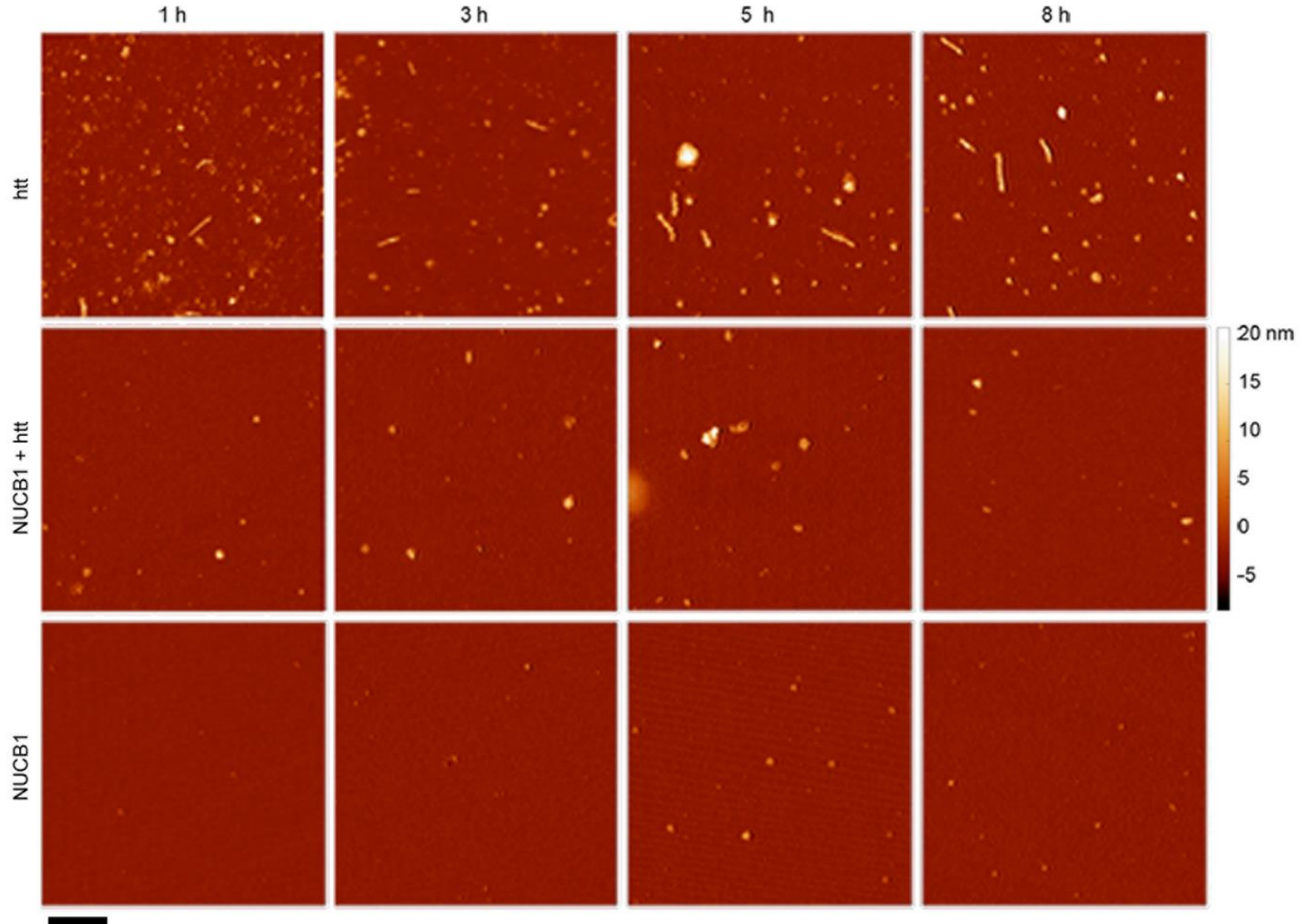

$500 \mathrm{~nm}$

Figure 4.4. NUCB1 inhibited the aggregation of htt-exon1. Htt-exon1(46Q) (10 $\mu M)$ with and without NUCB1 $(10 \mu \mathrm{M})$ were incubated for $8 \mathrm{~h}$. Aliquots of incubations were taken at various time points (1,3,5 and 8 h), deposited on mica and imaged with AFM. 
$\alpha$-synuclein interacts with $\beta$-synuclein, which it colocalizes with. ${ }^{57}$ Such interaction impeded the self-assembly of $\alpha$-synuclein..$^{57}$ In addition, the kinetics of aggregation can also be altered through protein-protein interactions. ${ }^{58} \beta$-amyloid, one of the proteins implicated in $A D$, can seed, and promote the aggregation of $\alpha$-synuclein. ${ }^{59}$ For all these reasons, determining how protein-protein interaction influence aggregation may prove important to understanding associated toxic mechanisms.

Nucleobindin1 (NUCB1) is a Golgi-resident protein that binds and controls calcium homeostasis. ${ }^{60} \mathrm{~A}$ non-calcium binding variant of NUCB1 has been shown to reduce amyloid formation in amyloidogenic proteins such as Human islet amyloid polypeptide ${ }^{61}$ and $\alpha$-synuclein ${ }^{62}$ by stabilizing the protofibrils, or short soluble filamentous species which are commonly formed by amyloid proteins. Therefore, it is plausible that NUCB1 could

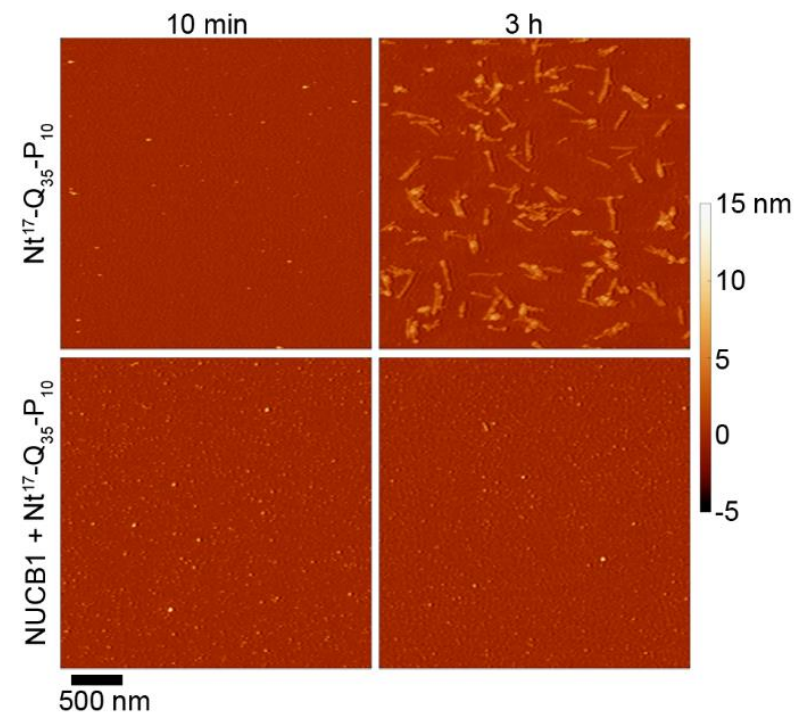

Figure 4.5. NUCB1 inhibited the aggregation of $\mathbf{N t}^{17}-\mathrm{Q}_{35}-\mathrm{P}_{10}-\mathrm{KK}$. Nt ${ }^{17}-\mathrm{Q}_{35}-\mathrm{P}_{10}-\mathrm{KK}(10$ $\mu M)$ was incubated with and without NUCB1 $(10 \mu \mathrm{M})$ for $3 \mathrm{~h}$. Aliquots from incubations drawn after 10 min and $3 h$ were deposited on mica and imaged with AFM. 


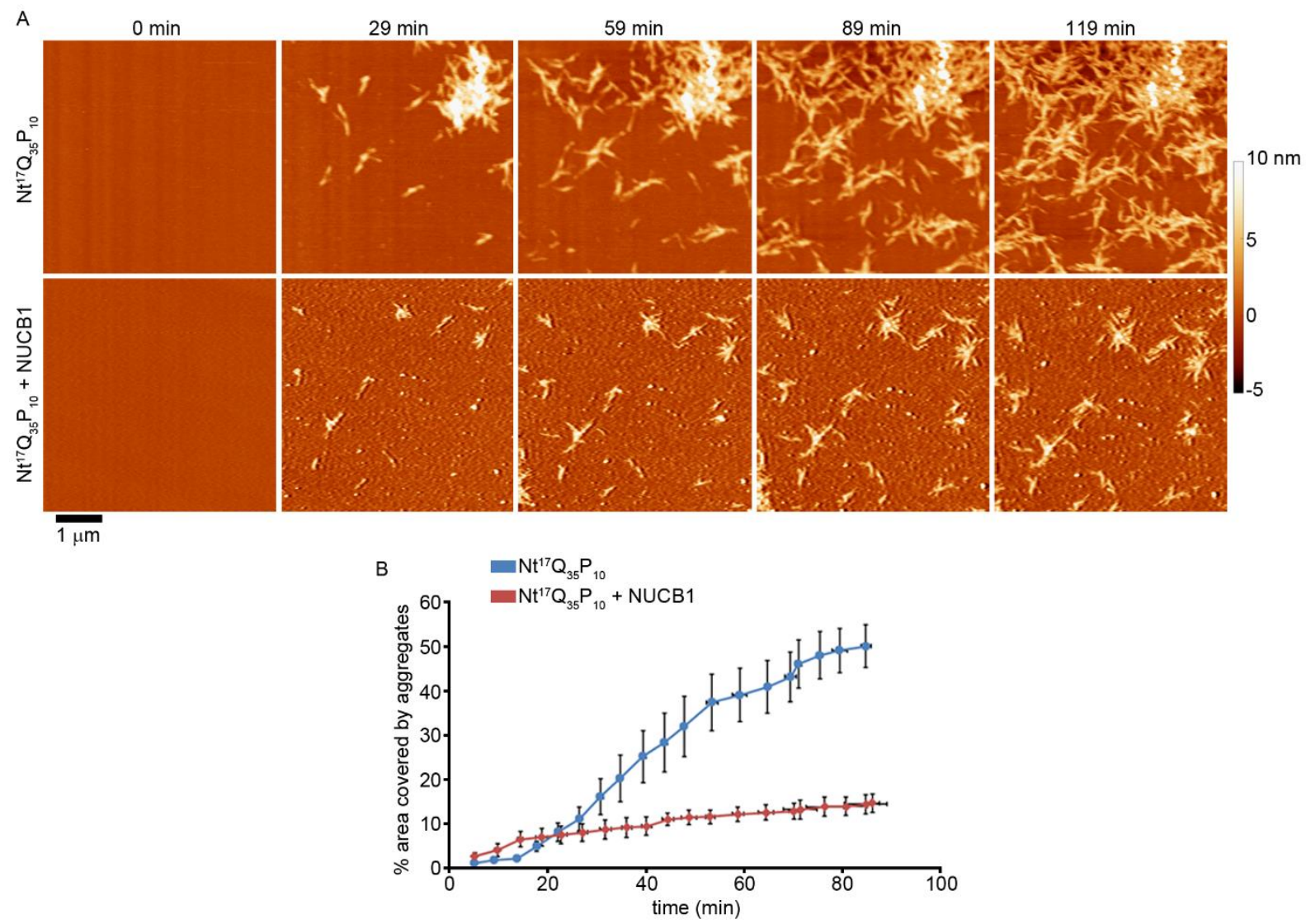

Figure 4.6. In situ tracking of $\mathrm{Nt}^{17}-\mathrm{Q}_{35}-\mathrm{P}_{10}-\mathrm{KK}$ in the presence of NUCB1. (A) $\mathrm{Nt}^{17}$ $Q_{35}-P_{10}-K K(10 \mu M)$ was incubated with and without NUCB1 $(10 \mu M)$ and imaged continuously for $119 \mathrm{~min}$. (B) Analysis of the surface area covered by $N t^{17}-Q_{35}-P_{10}-K K$ in the presence and absence of NUCB1.

have a similar aggregation inhibiting effect on htt. The validity of this hypothesis is supported by preliminary studies. First, htt-exon1 incubated with NUCB1 displayed a clear reduction in the amount of fibrils formed (Figure 4.4). Second, a similar study of incubations of htt-exon1 mimic (Nt $\left.{ }^{17}-\mathrm{Q}_{35}-\mathrm{P}_{10}-\mathrm{KK}\right)$ and NUCB1 showed no fibrils formed after $3 \mathrm{~h}$ of incubation with even though $\mathrm{Nt}^{17}-\mathrm{Q}_{35}-\mathrm{P}_{10}-\mathrm{KK}$ aggregated in the absence of NUCB1 was significant at similar time point (Fig 4.5). Third, in situ AFM studies tracking the aggregation of the synthetic $h t t$ mimic peptide $\left(\mathrm{Nt}^{17}-\mathrm{Q}_{35}-\mathrm{P}_{10}-\mathrm{KK}\right)$ on mica revealed a 
clear reduction of aggregation (Figure 4.6). This demonstrates that protein-protein interactions represent another mitigating factor in htt aggregation.

\subsection{Concluding remarks}

The findings presented in this work demonstrate the relevance of membranous surfaces and chemical modifications to htt aggregation and HD. We showed that AFM is a valuable technique to study the morphology of aggregates derived from either exposing htt to various membranes or subjecting htt to post translational modifications. It was shown that the aggregation of htt is influenced not only by the presence of membranes, but that composition of membranes also play an important role. Furthermore, we also revealed that oxidation promoted oligomerization and distinct fibrillar morphology, both of which provide insights into the role of increased oxidative environment in HD pathogenesis.

The ideas and results presented in this chapter further emphasized the complexity of htt aggregation and presented possible ways of mitigating it. Specifically, it was suggested that $\mathrm{CL}$ which inhibited aggregation may have similar effect on htt toxicity. In addition, it was demonstrated interactions of htt with other proteins or other macromolecules have a variety of effects on htt aggregation. These investigations and findings are directed towards understanding and inhibiting htt aggregation as a potential therapeutic approach to HD. 


\subsection{References}

(1) Adegbuyiro, A., Sedighi, F., Pilkington, A. W., Groover, S., and Legleiter, J. (2017) Proteins Containing Expanded Polyglutamine Tracts and Neurodegenerative Disease. Biochemistry 56, 1199-1217.

(2) Legleiter, J., Mitchell, E., Lotz, G. P., Sapp, E., Ng, C., DiFiglia, M., Thompson, L. M., and Muchowski, P. J. (2010) Mutant huntingtin fragments form oligomers in a polyglutamine length-dependent manner in Vitro and in Vivo. Journal of Biological Chemistry 285, 14777-14790.

(3) Wetzel, R. (2012) Physical chemistry of polyglutamine: Intriguing tales of a monotonous sequence. Journal of Molecular Biology 421, 466-490.

(4) Saha, I., Mishra, A., Hartl, F. U., and Baumeister, W. (2017) In Situ Architecture and Cellular Interactions of PolyQ Inclusions Article In Situ Architecture and Cellular Interactions of PolyQ Inclusions. Cell 171, 179-187.

(5) Kim, Y. E., Hosp, F., Mann, M., Hayer-hartl, M., and Hartl, F. U. (2016) Soluble Oligomers of PolyQ-Expanded Huntingtin Target a Multiplicity of Key Cellular Factors Article Soluble Oligomers of PolyQ-Expanded Huntingtin Target a Multiplicity of Key Cellular Factors. Molecular Cell 63, 951-964.

(6) Nucifora, L. G., Burke, K. A., Feng, X., Arbez, N., Zhu, S., Miller, J., Yang, G., Ratovitski, T., Delannoy, M., Muchowski, P. J., Finkbeiner, S., Legleiter, J., Ross, C. A., and Poiriera, M. A. (2012) Identification of novel potentially toxic oligomers formed in vitro from mammalian-derived expanded huntingtin exon-1 protein. Journal of Biological Chemistry 287, 16017-16028.

(7) Lajoie, P., and Snapp, E. L. (2010) Formation and toxicity of soluble polyglutamine oligomers in living cells. PLOS ONE 5, e15245.

(8) Drombosky, K. W., Rode, S., Kodali, R., Jacob, T. C., Palladino, M. J., and Wetzel, R. (2018) Mutational analysis implicates the amyloid fibril as the toxic entity in Huntington's disease. Neurobiology of Disease 120, 126-138.

(9) Pieri, L., Madiona, K., Bousset, L., and Melki, R. (2012) Fibrillar a -Synuclein and Huntingtin Exon 1 Assemblies Are Toxic to the Cells. Biophysj 102, 2894-2905.

(10) Panov, A. v., Gutekunst, C.-A., Leavitt, B. R., Hayden, M. R., Burke, J. R., Strittmatter, W. J., and Greenamyre, J. T. (2002) Early mitochondrial calcium defects in Huntington's disease are a direct effect of polyglutamines. Nature Neuroscience 5, 731736.

(11) Orr, A. L., Li, S., Wang, C.-E., Li, H., Wang, J., Rong, J., Xu, X., Mastroberardino, P. G., Greenamyre, J. T., and Li, X.-J. (2008) N-Terminal Mutant Huntingtin Associates with 
Mitochondria and Impairs Mitochondrial Trafficking. Journal of Neuroscience 28, 27832792.

(12) Bossy-Wetzel, E., Petrilli, A., and Knott, A. B. (2008) Mutant huntingtin and mitochondrial dysfunction. Trends in Neurosciences 31, 609-616.

(13) Song, W., Chen, J., Petrilli, A., Liot, G., Klinglmayr, E., Zhou, Y., Poquiz, P., Tjong, J., Pouladi, M. A., Hayden, M. R., Masliah, E., Ellisman, M., Rouiller, I., Schwarzenbacher, R., Bossy, B., Perkins, G., and Bossy-Wetzel, E. (2011) Mutant huntingtin binds the mitochondrial fission GTPase dynamin-related protein-1 and increases its enzymatic activity. Nature medicine 17, 377-82.

(14) Yano, H., Baranov, S. V., Baranova, O. V., Kim, J., Pan, Y., Yablonska, S., Carlisle, D. L., Ferrante, R. J., Kim, A. H., and Friedlander, R. M. (2014) Inhibition of mitochondrial protein import by mutant huntingtin. Nature Neuroscience 17, 822-831.

(15) Pope, S., Land, J. M., and Heales, S. J. R. (2008) Oxidative stress and mitochondrial dysfunction in neurodegeneration; cardiolipin a critical target? Biochimica et Biophysica Acta - Bioenergetics 1777, 794-799.

(16) Zhou, C., Huang, Y., and Przedborski, S. (2008) Oxidative stress in Parkinson's disease: A mechanism of pathogenic and therapeutic significance, in Annals of the New York Academy of Sciences, pp 93-104.

(17) Niedzielska, E., Smaga, I., Gawlik, M., Moniczewski, A., Stankowicz, P., Pera, J., and Filip, M. (2016) Oxidative Stress in Neurodegenerative Diseases. Molecular Neurobiology.

(18) Kumar, A., and Ratan, R. R. (2016) Oxidative Stress and Huntington's Disease: The Good, the Bad, and the Ugly. Journal of Huntington's Disease.

(19) Chung, H. S., Wang, S. B., Venkatraman, V., Murray, C. I., and Van Eyk, J. E. (2013) Cysteine oxidative posttranslational modifications: Emerging regulation in the cardiovascular system. Circulation Research 112, 382-392.

(20) Waszczak, C., Akter, S., Jacques, S., Huang, J., Messens, J., and Van Breusegem, F. (2015) Oxidative post-translational modifications of cysteine residues in plant signal transduction. Journal of Experimental Botany 66, 2923-2934.

(21) Hoshi, T., and Heinemann, S. H. (2001) Regulation of cell function by methionine oxidation and reduction. Journal of Physiology 531, 1-11.

(22) Groover, S. E., Beasley, M., Ramamurthy, V., and Legleiter, J. (2020) Phosphomimetic Mutations Impact Huntingtin Aggregation in the Presence of a Variety of Lipid Systems. Biochemistry 59, 4681-4693.

(23) Chaibva, M., Jawahery, S., Pilkington, A. W., Arndt, J. R., Sarver, O., Valentine, S., Matysiak, S., and Legleiter, J. (2016) Acetylation within the First 17 Residues of 
Huntingtin Exon 1 Alters Aggregation and Lipid Binding. Biophysical Journal 111, 349362.

(24) Sedighi, F., Adegbuyiro, A., and Legleiter, J. (2020) SUMOylation Prevents Huntingtin Fibrillization and Localization onto Lipid Membranes. ACS Chemical Neuroscience 11, 328-343.

(25) Kagan, V. E., Jiang, J., Huang, Z., Tyurina, Y. Y., Desbourdes, C., Cottet-Rousselle, C., Dar, H. H., Verma, M., Tyurin, V. A., Kapralov, A. A., Cheikhi, A., Mao, G., Stolz, D., St Croix, C. M., Watkins, S., Shen, Z., Li, Y., Greenberg, M. L., Tokarska-Schlattner, M., Boissan, M., Lacombe, M. L., Epand, R. M., Chu, C. T., Mallampalli, R. K., Bayir, H., and Schlattner, U. (2016) NDPK-D (NM23-H4)-mediated externalization of cardiolipin enables elimination of depolarized mitochondria by mitophagy. Cell Death and Differentiation 23, $1140-1151$.

(26) Chu, C. T., Ji, J., Dagda, R. K., Jiang, J. F., Tyurina, Y. Y., Kapralov, A. A., Tyurin, V. A., Yanamala, N., Shrivastava, I. H., Mohammadyani, D., Qiang Wang, K. Z., Zhu, J., Klein-Seetharaman, J., Balasubramanian, K., Amoscato, A. A., Borisenko, G., Huang, Z., Gusdon, A. M., Cheikhi, A., Steer, E. K., Wang, R., Baty, C., Watkins, S., Bahar, I., Bayir, H., and Kagan, V. E. (2013) Cardiolipin externalization to the outer mitochondrial membrane acts as an elimination signal for mitophagy in neuronal cells. Nature Cell Biology 15, 1197-1205.

(27) Ostrander, D. B., Sparagna, G. C., Amoscato, A. A., McMillin, J. B., and Dowhan, W. (2001) Decreased Cardiolipin Synthesis Corresponds with Cytochrome c Release in Palmitate-induced Cardiomyocyte Apoptosis. Journal of Biological Chemistry 276, 38061-38067.

(28) Schlame, M., Kelley, R. I., Feigenbaum, A., Towbin, J. A., Heerdt, P. M., Schieble, T., Wanders, R. J. A., DiMauro, S., and Blanck, T. J. J. (2003) Phospholipid Abnormalities in Children with Barth Syndrome. Journal of the American College of Cardiology 42, 1994-1999.

(29) Kiebish, M. A., Han, X., Cheng, H., Chuang, J. H., and Seyfried, T. N. (2008) Cardiolipin and electron transport chain abnormalities in mouse brain tumor mitochondria: Lipidomic evidence supporting the Warburg theory of cancer. Journal of Lipid Research 49, 2545-2556.

(30) Lesnefsky, E. J., Minkler, P., and Hoppel, C. L. (2009) Enhanced modification of cardiolipin during ischemia in the aged heart. Journal of Molecular and Cellular Cardiology 46, 1008-1015.

(31) Saini-Chohan, H. K., Holmes, M. G., Chicco, A. J., Taylor, W. A., Moore, R. L., McCune, S. A., Hickson-Bick, D. L., Hatch, G. M., and Sparagna, G. C. (2009) Cardiolipin biosynthesis and remodeling enzymes are altered during development of heart failure. Journal of Lipid Research 50, 1600-1608. 
(32) Monteiro-Cardoso, V. F., Oliveira, M. M., Melo, T., Domingues, M. R. M., Moreira, P. I., Ferreiro, E., Peixoto, F., and Videira, R. A. (2014) Cardiolipin profile changes are associated to the early synaptic mitochondrial dysfunction in Alzheimer's disease. Journal of Alzheimer's Disease 43, 1375-1392.

(33) Chicco, A. J., and Sparagna, G. C. (2007) Role of cardiolipin alterations in mitochondrial dysfunction and disease. American Journal of Physiology - Cell Physiology 292, 33-44.

(34) Yan, J., Jiang, J., He, L., and Chen, L. (2020) Mitochondrial superoxide/hydrogen peroxide: An emerging therapeutic target for metabolic diseases. Free Radical Biology and Medicine 152, 33-42.

(35) Wong, H. S., Dighe, P. A., Mezera, V., Monternier, P. A., and Brand, M. D. (2017) Production of superoxide and hydrogen peroxide from specific mitochondrial sites under different bioenergetic conditions. Journal of Biological Chemistry 292, 16804-16809.

(36) Bulteau, A. L., Szweda, L. I., and Friguet, B. (2006) Mitochondrial protein oxidation and degradation in response to oxidative stress and aging. Experimental Gerontology 41, 653-657.

(37) Ademowo, O. S., Dias, H. K. I., Burton, D. G. A., and Griffiths, H. R. (2017) Lipid (per) oxidation in mitochondria: an emerging target in the ageing process? Biogerontology 18, 859-879.

(38) Cui, H., Kong, Y., and Zhang, H. (2012) Oxidative Stress, Mitochondrial Dysfunction, and Aging. Journal of Signal Transduction 2012, 1-14.

(39) Osman, C., Voelker, D. R., and Langer, T. (2011) Making heads or tails of phospholipids in mitochondria. Journal of Cell Biology 192, 7-16.

(40) Haluska, C. K., Baptista, M. S., Fernandes, A. U., Schroder, A. P., Marques, C. M., and Itri, R. (2012) Photo-activated phase separation in giant vesicles made from different lipid mixtures. Biochimica et Biophysica Acta - Biomembranes 1818, 666-672.

(41) Yusupov, M., Wende, K., Kupsch, S., Neyts, E. C., Reuter, S., and Bogaerts, A. (2017) Effect of head group and lipid tail oxidation in the cell membrane revealed through integrated simulations and experiments. Scientific Reports 7, 1-14.

(42) Wong-Ekkabut, J., Xu, Z., Triampo, W., Tang, I. M., Tieleman, D. P., and Monticelli, L. (2007) Effect of lipid peroxidation on the properties of lipid bilayers: A molecular dynamics study. Biophysical Journal 93, 4225-4236.

(43) Keller, A., Fritzsche, M., Yu, Y. P., Liu, Q., Li, Y. M., Dong, M., and Besenbacher, F. (2011) Influence of hydrophobicity on the surface-catalyzed assembly of the islet amyloid polypeptide. ACS Nano 5, 2770-2778. 
(44) Zhu, M., Souillac, P. O., lonescu-Zanetti, C., Carter, S. A., and Fink, A. L. (2002) Surface-catalyzed amyloid fibril formation. Journal of Biological Chemistry 277, 5091450922.

(45) Kowalewski, T., and Holtzman, D. M. (1999) In situ atomic force microscopy study of Alzheimer's $\beta$-amyloid peptide on different substrates: New insights into mechanism of $\beta$ sheet formation. Proceedings of the National Academy of Sciences of the United States of America 96, 3688-3693.

(46) Giacomelli, C. E., and Norde, W. (2003) Influence of hydrophobic teflon particles on the structure of amyloid $\beta$-peptide. Biomacromolecules 4, 1719-1726.

(47) Ha, C., and Park, C. B. (2006) Ex situ atomic force microscopy analysis of $\beta$-amyloid self-assembly and deposition on a synthetic template. Langmuir 22, 6977-6985.

(48) Morriss-Andrews, A., and Shea, J. E. (2012) Kinetic pathways to peptide aggregation on surfaces: The effects of -sheet propensity and surface attraction. Journal of Chemical Physics 136, 1-11.

(49) Burke, K. A., Yates, E. A., and Legleiter, J. (2013) Biophysical insights into how surfaces, including lipid membranes, modulate protein aggregation related to neurodegeneration. Frontiers in Neurology 4, 1-17.

(50) Sarkar, M., Li, C., and Pielak, G. J. (2013) Soft interactions and crowding. Biophysical Reviews 5, 187-194.

(51) Minton, A. P. (2000) Implications of macromolecular crowding for protein assembly. Current Opinion in Structural Biology 10, 34-39.

(52) Ellis, R. J. (2001) Macromolecular crowding: Obvious but underappreciated. Trends in Biochemical Sciences 26, 597-604.

(53) Latshaw, D. C., and Hall, C. K. (2015) Effects of Hydrophobic Macromolecular Crowders on Amyloid $\beta$ (16-22) Aggregation. Biophysical Journal 109, 124-134.

(54) Uversky, V. N., M. Cooper, E., Bower, K. S., Li, J., and Fink, A. L. (2002) Accelerated a-synuclein fibrillation in crowded milieu. FEBS Letters 515, 99-103.

(55) Hatters, D. M., Minton, A. P., and Howlett, G. J. (2002) Macromolecular crowding accelerates amyloid formation by human apolipoprotein C-II. Journal of Biological Chemistry 277, 7824-7830.

(56) Kuznetsova, I. M., Turoverov, K. K., and Uversky, V. N. (2014) What macromolecular crowding can do to a protein. International Journal of Molecular Sciences 15, 2309023140.

(57) Janowska, M. K., Wu, K. P., and Baum, J. (2015) Unveiling transient protein-protein interactions that modulate inhibition of alpha-synuclein aggregation by beta-synuclein, a pre-synaptic protein that co-localizes with alpha-synuclein. Scientific Reports 5, 1-10. 
(58) Ghosh, R., Calero-Rubio, C., Saluja, A., and Roberts, C. J. (2016) Relating ProteinProtein Interactions and Aggregation Rates from Low to High Concentrations. Journal of Pharmaceutical Sciences 105, 1086-1096.

(59) Tsigelny, I. F., Crews, L., Desplats, P., Shaked, G. M., Sharikov, Y., Mizuno, H., Spencer, B., Rockenstein, E., Trejo, M., Platoshyn, O., Yuan, J. X. J., and Masliah, E. (2008) Mechanisms of hybrid oligomer formation in the pathogenesis of combined Alzheimer's and Parkinson's diseases. PLOS ONE 3, 1-15.

(60) Lin, P., Le-Niculescu, H., Hofmeister, R., McCaffery, J. M., Jin, M., Hennemann, H., McQuistan, T., De Vries, L., and Farquhar, M. G. (1998) The mammalian calcium-binding protein, nucleobindin (CALNUC), is a Golgi resident protein. Journal of Cell Biology 141, $1515-1527$.

(61) Gupta, R., Kapoor, N., Raleigh, D. P., and Sakmar, T. P. (2012) Nucleobindin 1 caps human islet amyloid polypeptide protofibrils to prevent amyloid fibril formation. Journal of Molecular Biology 421, 378-389.

(62) Bonito-Oliva, A., Barbash, S., Sakmar, T. P., and Graham, W. V. (2017) Nucleobindin 1 binds to multiple types of pre-fibrillar amyloid and inhibits fibrillization. Scientific Reports 7, 1-12. 